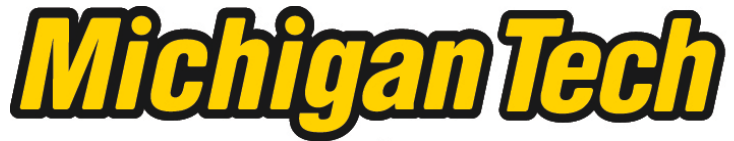 \\ Michigan Technological University Create the Future Digital Commons @ Michigan Tech
}

Dissertations, Master's Theses and Master's Reports - Open

Dissertations, Master's Theses and Master's

Reports

2005

\section{Control algorithms for large scale adaptive optics}

Piotr Piatrou

Michigan Technological University

Follow this and additional works at: https://digitalcommons.mtu.edu/etds

Part of the Electrical and Computer Engineering Commons

Copyright 2005 Piotr Piatrou

\section{Recommended Citation}

Piatrou, Piotr, "Control algorithms for large scale adaptive optics", Dissertation, Michigan Technological University, 2005.

https://doi.org/10.37099/mtu.dc.etds/66

Follow this and additional works at: https://digitalcommons.mtu.edu/etds

Part of the Electrical and Computer Engineering Commons 


\section{Michigan Technological University}

Houghton, MI

\section{Control Algorithms for Large Scale Adaptive Optics}

A dissertation submitted in partial fulfillment of

the requirements for the degree of

Doctor of Philosophy

(Electrical \& Computer Engineering)

by

Piotr Piatrou

2005 
(C) Copyright by

Piotr Piatrou

2005 
The dissertation of Piotr Piatrou is approved.

Michael C. Roggemann

Timothy J. Schulz

Jeffrey B. Burl

Alex Kostinski

Michael C. Roggemann, Committee Chair

Michigan Technological University, Houghton, MI

2005 


\section{TABle of Contents}

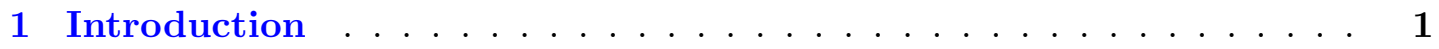

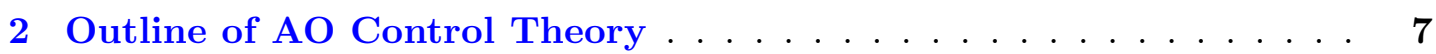

2.1 Idea of adaptive compensation of turbulence-induced optical aberrations. $\quad 7$

2.2 Atmospheric turbulence theory. . . . . . . . . . . . . . . . 8

2.3 Model for turbulence temporal dynamics. . . . . . . . . . . . . . . . . 13

2.4 Image quality assessment. . . . . . . . . . . . . . . 15

2.5 Factors limiting AO system performance. . . . . . . . . . . . . 17

2.5 .1 Anisoplanatism. . . . . . . . . . . . . . . . 18

2.5.2 Temporal error. . . . . . . . . . . . . . . . . 20

2.5.3 Measurement error. . . . . . . . . . . . . . . . 20

2.5 .4 DM fitting error. . . . . . . . . . . . . . . . 22

2.5 .5 Other sources of errors. . . . . . . . . . . . . . 22

2.6 Multi Conjugate AO . . . . . . . . . . . . . . . . . . . . 24

3 Mathematical modeling of AO systems. . . . . . . . . . . . 29

3.1 Model for atmosphere. . . . . . . . . . . . . . . . . . . . . 29

3.2 Wave front sensor model. . . . . . . . . . . . . . . . . 37

3.3 Deformable mirror model. . . . . . . . . . . . . . . . . . . 40

3.4 Temporal discretization. . . . . . . . . . . . . . . . . 41

3.5 Performance estimation. . . . . . . . . . . . . . . . . 42 
3.6 Phase reconstructors. . . . . . . . . . . . . . . . . . . . 47

3.6.1 Least Squares (LS) reconstructor. . . . . . . . . . . . . . . . 48

3.6.2 Minimum Variance reconstructor. . . . . . . . . . . . . . . . 49

3.6.3 Kalman Filter. . . . . . . . . . . . . . . . . . . . . 50

3.7 Monte-Carlo simulations. . . . . . . . . . . . . . . . . . 52

4 Robustness study of the sparse non-dynamic Minimum Variance con-

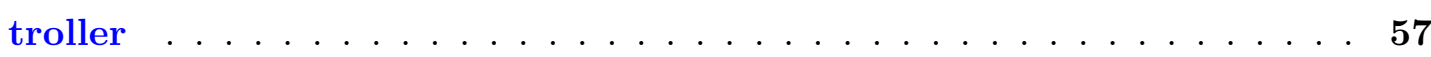

4.1 Low complexity implementation of the MV reconstruction algorithm. . . 58

4.1.1 Sparse approximation of matrix $C_{\phi \phi}^{-1} \ldots \ldots \ldots \ldots$

$4.1 .2 \quad$ Fast matrix inverses. . . . . . . . . . . . . . . . . 64

4.2 Closed-loop operation.

Idea of the Pseudo Open Loop MV controller. . . . . . . . . . . . . . 68

4.3 Pseudo Open Loop Control robustness

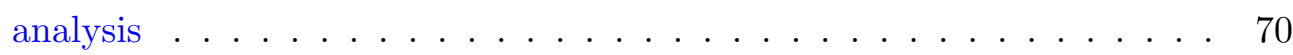

4.4 Simulation results . . . . . . . . . . . . . . . . 73

4.4 .1 Stability analysis results . . . . . . . . . . . . 81

4.4 .2 Performance analysis results . . . . . . . . . . . . . . 81

4.5 Robustness analysis conclusions. . . . . . . . . . . . . . . . 84

5 Performance Analysis of Minimum Variance and Kalman Filter Con-

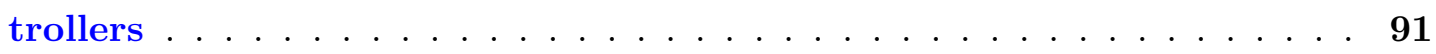

5.1 Near-Markov turbulence model. . . . . . . . . . . . . . . . . . . . . . . 92

$5.2 \quad$ Kalman Filter equations. . . . . . . . . . . . . . . . . . . . . . . 94 
5.3 Simulation results. . . . . . . . . . . . . . . . . . . . 95

5.4 Conclusions. . . . . . . . . . . . . . . . . . . . . . 102

6 Sparse Predictive Minimum Variance Controller. . . . . . . . . . . 103

6.1 Idea of the sparse phase prediction. . . . . . . . . . . . . . . 103

6.2 Simulation results. . . . . . . . . . . . . . . . . . 106

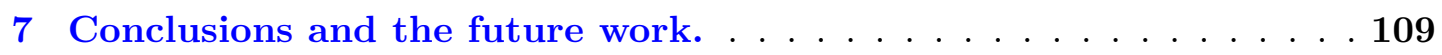

Appendix A ........................ 115

Appendix B .......................... 119

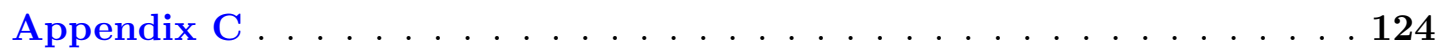

References . . . . . . . . . . . . . . . . . . 126 


\section{List OF FiguRES}

2.1 Setup of the conventional adaptive optics system. . . . . . . . . . . . 9

2.2 Angular (a) and focal (b) anisoplanatism. . . . . . . . . . . 18

2.3 Multi Conjugate Adaptive Optics system layout. . . . . . . . . . . . 25

3.1 Cerro Pachon turbulence profile and its 6-layer discretization (shown with diamonds). Left panel: $C_{n}^{2}$ profile. Right panel: wind velocity profile. 31

4.1 Sparsity patterns of the $G_{a^{-}}(\mathrm{a}), H_{a^{-}}$(b) and $W$-matrices (c) of the model for the Gemini South 8-meter telescope MCAO system. The filling factors are $2.49 \%, 0.07 \%$ and $1.5 \%$, respectively. . . . . . . 59

4.2 Discrete Laplacian or curvature operator [70]. This figure illustrates the coefficients of discrete Laplacian computation. Stars represent the grid points of the discrete phase grid. The values near the stars are the nonzero coefficients needed to compute the value of Laplacian at the interior point $\mathrm{A}$ or the boundary points B, C. . . . . . . . . 63

$4.3 z$-domain block diagrams of non-ideal MCAO system driven by POLC. . 72

4.4 Subaperture arrangement in LGS wave front sensor. 224 active subapertures fill the main aperture. . . . . . . . . . . . . . 75

4.5 Representative Gemini-South $8 \mathrm{~m}$ telescope MCAO system guide stars arrangement and scientific field of view $(\mathrm{ScFoV}) \ldots \ldots \ldots \ldots$

4.6 Deformable mirrors actuator grids. Active actuators are shown with crosses. The circles are the main aperture projections onto DMs along the scientific directions. . . . . . . . . . . . . 76 
4.7 Reconstruction phase grids on the six phase screens, whose parameters are given in Table 4.3. Grid points positions are shown with crosses. The circles are the main aperture projections onto the phase screens along the scientific and guide stars directions. . . . . . . . . . . 78

4.8 Closed-loop (circles) and rejection (triangles) power spectra of the servo compensator used in the system. . . . . . . . . . . . . . 79

4.9 Transfer function poles evolution as a function of the DMs shift misalignment value. Each frame gives positions of the closed-loop system poles for different degree of misalignment. All three DMs are shifted simultaneously from their perfect alignment position in directions that make a $120 \mathrm{deg}$ angle with one another. . . . . . . . . . . . 82

4.10 Wave front phase error variance time histories for a number of shift misalignments levels in DMs (upper panel) and WFSs (lower panel). DM shift directions make a 120 deg angle with one another. WFS shift directions make a $45 \mathrm{deg}$ angle with one another. . . . . . . . .

4.11 Wave front phase error time histories for a number of rotation misalignment levels in DMs (upper panel) and WFSs (lower panel). Rotations in each DM are opposite to one another. All WFSs are rotated clockwise. 86

4.12 Wave front phase error time histories for different levels of mismagnification in DMs (upper panel) and WFSs (lower panel). Mismagnifications are set equal for each DM or WFS. . . . . . . . . . . . . . . . 8

4.13 Wave front phase error time histories for different tilt levels in DMs (upper panel) and WFSs (lower panel). Tilt directions in each DM make a 120 deg angle with one another. Tilt directions in each WFS make a $45 \mathrm{deg}$ angle with one another. . . . . . . . . . . . . 88 
4.14 Wave front phase error time histories for different levels of additive noise in the DM actuator command vector. White Gaussian noise is assumed. The noise level is given as a fraction of the largest actuator command vector component at each time step. . . . . . . . . . . .

5.1 Turbulence phase prediction error as a function of delayed samples number for the near-Markov model computed by Eq. (5.13). Piston-removed Kolmogorov turbulence with Fried parameter $r_{0}=0.166 \mathrm{~m}$ is assumed. Wind speed is equal to $|\vec{v}|=10 \mathrm{~m} / \mathrm{s}$, the system sampling rate is $f=100$ Hz. A single discrete phase screen with 248 equidistant phase grid points fill the 5.1-meter circular pupil of the Palomar telescope. . . . . . . . . . 96

5.2 Guide stars (left panel) and field of view (right panel) alignment for Gemini South MCAO system. . . . . . . . . . . . . . . . . . 97

5.3 Left panel: fifth phase screen reconstruction phase grid and beamprint. The circles are projections of the annular aperture onto the screen along both scientific and guide stars directions. Right panel: LGS WFS subaperture geometry for Gemini South MCAO. Big and small circles are the outer and inner borders of the annular main aperture of the system.

5.4 Estimation error time evolution graphs obtained by Monte-Carlo simulations of the Palomar (left panel) and Gemini South (right panel) AO systems. Sampling rate $500 \mathrm{~Hz}$. The KF curves correspond to the case of 2-step prediction, whereas MV curves correspond to pure 2-step lag. . 100

6.1 Illustration of the guard band addition to the phase grid. (a): initial position of the grid. Main grid points are denoted by “*”. Guard band points are denoted by " $\mathrm{x}$ ". (b): grid position after a wind shift. . . . . . 105 
$6.2 z$-domain block diagram of the MV Pseudo Open Loop Controller with the sparse predictor. . . . . . . . . . . . . 106

6.3 Reconstruction error time evolution graphs obtained by Monte-Carlo simulations of the Palomar (left panel) and Gemini South (right panel, center of FoV) $\mathrm{AO}$ systems driven by sparse MV controller with 2-step prediction turned on/off. . . . . . . . . . . . . . . . . . . 108

7.1 Mutual alignment of a WFS subaperture and a bilinear spline. $W_{s u b}^{i}, W_{h}^{j}$ are the domains occupied by a $i^{\text {th }}$ subaperture and $j^{\text {th }}$ spline, respectively 120

7.2 Illustration of the numerical integration method. . . . . . . . . . . . 122 


\section{List OF TABLES}

4.1 Wave front sensors of the Gemini-South $8 \mathrm{~m}$ telescope MCAO system. . 74

4.2 Gemini-South $8 \mathrm{~m}$ telescope MCAO system deformable mirror parameters. 77

4.3 Six-layer turbulence model used in the simulation of the Gemini-South $8 \mathrm{~m}$ telescope MCAO system. . . . . . . . . . . . . . . . . 79

4.4 Approximate stability margins for the various misalignments in DMs for LSC (first number) and for POLC (second number). Single-mirror shifts are done in $45 \mathrm{deg}$ direction, i.e. $\mathrm{x}$-component of the shift is equal to the y-component. Three-mirror shifts are done such that the shift directions of the mirrors make an angle 120 deg with one another. Three-mirror rotations are done such that the rotation directions of the mirrors are opposite to one another. Mismagnification is the same for each DM. . . 83

4.5 Approximate stability margins for misalignments in WFSs. Shift directions in WFSs make a 120 deg angle with one another. WFS rotation is done clockwise in all sensors. WFS mismagnification is the same for each sensor. . . . . . . . . . . . . . . . . 83

4.6 Average Strehl ratios obtained for various types and degrees of system errors. In each triad first, second and third numbers correspond, respectively, to the center, right side and upper right corner of the field of view. The statistical error in the Strehl ratios computation does not exceed $10 \%$ in all cases. . . . . . . . . . . . . . . . . . 90

5.1 Cerro Pachon 7-layer turbulence model. . . . . . . . . . . . . . . . . . 98 
5.2 Estimation errors (in nanometers rms) for Palomar AO system averaged over simulation time for $\mathrm{KF}$ and MV with 2-step prediction switched on/off. . . . . . . . . . . . . . . . . . . . . . 101

5.3 Estimation errors (in nanometers rms, center/side/corner of FoV) for Gemini South AO system averaged over simulation time for KF and MV with 2 -step prediction switched on/off. . . . . . . . . . . . . . . . 101

6.1 Reconstruction errors (in nanometers rms) for Palomar and Gemini South AO systems averaged over simulation time with 2-step prediction switched on/off. . . . . . . . . . . . . . . . . . . 108 


\section{ACKNOWLEDGMENTS}

This work has been supported by the National Science Foundation and Technology Center for Adaptive Optics, managed by the University of California at Santa Cruz under cooperative agreement No. AST-9876783.

The author wants to especially acknowledge professors Michael Roggemann and Jeffrey Burl for helpful discussions and support. 


\section{Abstract of the Dissertation \\ Control Algorithms for Large Scale Adaptive Optics}

by

\section{Piotr Piatrou}

Doctor of Philosophy in Electrical \& Computer Engineering

Michigan Technological University, Houghton, 2005

In this dissertation, the problem of creating effective large scale Adaptive Optics (AO) systems control algorithms for the new generation of giant optical telescopes is addressed. The effectiveness of $\mathrm{AO}$ control algorithms is evaluated in several respects, such as computational complexity, compensation error rejection and robustness, i.e. reasonable insensitivity to the system imperfections. The results of this research are summarized as follows:

1. Robustness study of Sparse Minimum Variance Pseudo Open Loop Controller (POLC) for multi-conjugate adaptive optics (MCAO). The AO system model that accounts for various system errors has been developed and applied to check the stability and performance of the POLC algorithm, which is one of the most promising approaches for the future AO systems control. It has been shown through numerous simulations that, despite the initial assumption that the exact system knowledge is necessary for the POLC algorithm to work, it is highly robust against various system errors.

2. Predictive Kalman Filter (KF) and Minimum Variance (MV) control algorithms for MCAO. The limiting performance of the non-dynamic Minimum Variance and 
dynamic KF-based phase estimation algorithms for MCAO has been evaluated by doing Monte-Carlo simulations. The validity of simple near-Markov autoregressive phase dynamics model has been tested and its adequate ability to predict the turbulence phase has been demonstrated both for single- and multiconjugate AO. It has also been shown that there is no performance improvement gained from the use of the more complicated KF approach in comparison to the much simpler MV algorithm in the case of MCAO.

3. Sparse predictive Minimum Variance control algorithm for MCAO. The temporal prediction stage has been added to the non-dynamic MV control algorithm in such a way that no additional computational burden is introduced. It has been confirmed through simulations that the use of phase prediction makes it possible to significantly reduce the system sampling rate and thus overall computational complexity while both maintaining the system stable and effectively compensating for the measurement and control latencies. 


\section{CHAPTER 1}

\section{Introduction}

Astronomy is one of the oldest and the most exciting branches of fundamental science, which investigates matter and energy in the universe through direct observations with the aid of various astronomical instruments. Astronomy is an instrument-based science and its progress is closely connected to the progress in design of astronomical instruments for all kinds of sensible radiation. Recent progress in the field of optics and photonics provides scientists the opportunity to obtain in near future optical astronomical instruments with new outstanding parameters, which are very likely to lead to new scientific discoveries. Several countries are preparing for deployment of the new generation of giant ground based optical telescopes with mirror diameters ranging from 20 to 100 meters equipped with the new high resolution image and spectral sensors. A few of the most important projects in this area are:

- Thirty meter telescope (TMT) [1]. Its first light is planned to be in 10-15 years. The telescope basic parameters are:

- primary mirror diameter is $30 \mathrm{~m}$;

- field of view is 20 arcmin,

- angular resolution at $1 \mu \mathrm{m}$ is 0.01 arcsec

The most likely location, where this telescope will be built is Mauna Kea, Hawaii. 
- 20/20 binocular telescope/interferometer [2]. Its first light is planned to be in 10-15 years. The telescope parameters are:

- diameter of two primary mirrors is $21 \mathrm{~m}$;

- angular resolution is $0.01 \operatorname{arcsec}$ at $1 \mu \mathrm{m}$;

The construction site is Las Campanas, Chile.

- Giant Magellan Telescope (GMT) [3]. Its first light is in 2016. The telescope parameters are:

- seven 8.4 meter circular segments make 25.4-meter overall diameter of the primary mirror;

- it is claimed to have ten times better resolution than that of Hubble telescope.

The construction site is Las Campanas, Chile.

- Overwhelmingly large telescope (OWL) [4]. The parameters are:

- primary mirror diameter is $100 \mathrm{~m}$;

- field of view is 3 arcmin;

- angular resolution is 0.003 arcsec;

Construction site is La Silla, Chile.

The advantage of the large ground-based optical telescopes over space telescopes is the possibility to use significantly bigger mirrors, which are currently impossible to launch into space. Since the collected energy is proportional to the square of the mirror diameter, the ground-based thirty-meter class telescope is able to collect over 100 times more power than the 2.4-meter Hubble space telescope, and a ground-based telescope 
construction and subsequent operation are about an order of magnitude cheaper than those of a space telescope. Also, ground-based telescopes allow greater versatility of instruments and have a much longer life time than space telescopes.

In addition to a number of engineering challenges of large telescope construction, such as making large stable and precise mirrors, extra-lightweight support structures, etc., a fundamental problem to overcome is the severe image quality deterioration due to atmospheric turbulence. Despite the fact that, if placed in vacuum, the diffraction limited angular resolution improves proportionally to the primary mirror diameter, the resolution of the system placed in air is limited by the atmospheric turbulence correlation radius (Fried parameter), which (in near infrared, the most interesting spectral region) does not exceed $0.5 \mathrm{~m}$ even for the best astronomical sites on Earth [6]. Only if this problem is effectively addressed will large ground-based telescopes become a really outstanding astronomical instrument.

Adaptive optics ( $\mathrm{AO}$ ) compensation of the aberrations induced by the atmosphere is believed to be the most effective technology to eliminate the effects of atmospheric turbulence in astronomical imaging. Adaptive optics is an interdisciplinary area, the confluence of optics, mathematics, control theory and advanced hardware engineering. Adaptive optics systems have become an integral part of many large optical telescopes, both existing ones and the ones planned to be built in the future. The progress made in the area for the last twenty years brought to life what is now called "conventional adaptive optics", the moderate size systems capable of compensating the turbulenceinduced aberration in the main aperture and achieving nearly diffraction-limited image quality within a-few-arc-second field of view. Conventional adaptive optics systems are now operating on a number of existing astronomical telescopes (see e.g. [61]) and have already shown the potential of adaptive optics to perform nearly diffractionlimited imaging in the presence of the atmosphere [5]. However, conventional adaptive 
optics is unable to deal with the problem of atmospheric turbulence compensation in the new giant telescopes. Among many other challenges the problem of creating the new effective AO system control algorithms is one of the least developed and the most important areas of adaptive optics, because almost all aspects of $\mathrm{AO}$ system performance are influenced to some extent by the control strategy chosen. The control algorithms normally used in existing $\mathrm{AO}$ systems are inadequate to serve for giant telescopes, and they do not reflect either modern control theory, or the state of the research on control algorithms for adaptive optics $[7,8,9,10,11,12]$. A list of the most important problems to be solved in the new generation of AO control algorithms includes:

1. Very high hardware complexity of the systems under consideration (typical number of degrees of freedom in these systems varies from $10^{4}$ to $10^{5}$ ).

2. Necessity to achieve the compensated field of view as large as a few tens of arcseconds.

3. Necessity to work with artificial beacons.

4. Necessity to achieve the most accurate atmospheric turbulence compensation possible with the available set of measurements.

5. Temporal prediction ability in order to compensate for time delays due to measurement readout and control command computation.

6. Necessity to come up with control strategies reasonably insensitive to various misalignments and calibration errors normally present in real AO systems.

The first problem in the list, that is the need to process in real time the information from of the order of $10^{5}$ channels, appears to be the most difficult. The current matrix 
multiplication algorithms normally have the computational complexity of $\mathscr{O}\left(N^{2}\right)$, where $N$ is the number of degrees of freedom, which is agreeable with the hardware cost and time considerations when $N \sim 10^{2}$ but makes the practical implementation of an AO system impossible, if $N \sim 10^{5}$. The development of control algorithms of the cost below $\mathscr{O}\left(N^{2}\right)$ requires not only new mathematical approaches to perform the calculations but also better understanding of the nature of atmospheric turbulence compensation.

This dissertation is the summary of the author's work on effective low complexity control algorithms for large scale AO systems intended for use in new very large optical telescopes. The author's contribution in this area of science can be split into three categories:

- Robustness study of Sparse Pseudo-Open-Loop Minimum Variance controller (POLC) for multi-conjugate adaptive optics (MCAO) [50]. This addresses the issues (1), (2) and (6) in the above list. The AO system model that accounts for various system errors has been developed and applied to check the stability and performance of the POLC algorithm, which is one of the most promising approaches for the future AO systems control. It has been shown through numerous simulations that, despite the initial assumption that the exact system knowledge is necessary for the POLC algorithm to work, it is highly robust against various system errors.

- Predictive Kalman Filter (KF) and Minimum Variance (MV) control algorithms for MCAO (issues (4) and (5)) [58]. The limiting performance of the non-dynamic MV and dynamic KF-based phase estimation algorithms for MCAO has been evaluated by doing Monte-Carlo simulations. The validity of simple near-Markov auto-regressive phase dynamics model has been tested and its adequate ability to predict the turbulence phase has been demonstrated both for single- and multiconjugate AO. It has also been shown that there is no performance improvement 
gained from the use of the more complicated KF approach in comparison to the much simpler MV algorithm in the case of MCAO.

- Sparse predictive Minimum Variance control algorithm for (MCAO) (issues (1) and (5)). The temporal prediction stage has been added to the non-dynamic MV control algorithm in such a way that no additional computational burden is introduced. It has been confirmed through simulations that the use of phase prediction makes it possible to significantly reduce the system sampling rate and thus overall computational complexity while both maintaining the system stable and effectively compensating for the measurement and control latencies.

The reminder of the dissertation is organized as follows.

Chapter 2 gives an overview of the basic ideas for AO system operation and current state of the research on AO control.

Chapter 3 gives the theoretical background necessary to analyze the various aspects of $\mathrm{AO}$ systems performance.

Chapter 4 gives an account of the robustness study of the Pseudo Open-Loop Controller.

Chapter 5 is devoted to the comparison of the predictive Kalman Filter and Minimum Variance control for MCAO.

Chapter 6 is an account of the most recent work on the Sparse Predictive Pseudo Open-Loop Minimum Variance Controller for MCAO.

Chapter 7 is the overview of possible progress in the area of control algorithms for large scale AO systems that can be done in the future. 


\section{CHAPTER 2}

\section{Outline of AO Control Theory}

\subsection{Idea of adaptive compensation of turbulence- induced optical aberrations.}

Light from a distant astronomical object, which is a superposition of plane waves, penetrates the Earth's atmosphere and gets distorted by it. Three major factors are recognized to contribute to atmospheric distortion of images:

1. Light refraction by the large scale, i.e. much larger than the observation wavelength, component of atmospheric turbulence.

2. Rayleigh scattering by the small scale, i.e. the order of the observation wavelength, fraction of turbulence and scattering by the water, ice or soot particles drifting in the atmosphere. The background light due to the scattering of the ground and celestial light sources by the atmosphere should also be mentioned.

3. Light attenuation due to the absorption of air gases, which does not deteriorate the sharpness of image but may significantly distort its spectral content.

The second and third distortion factors can be almost eliminated by the positioning an observatory at high altitude, far from big cities and, of course, by night observations for scattering elimination; operating in visible, near and mid-infrared, the spectral 
regions, where atmospheric absorption is small, the first factor still remains the fundamental obstacle for achieving the diffraction-limited quality images on ground-based telescopes. On the other hand, the nature of atmospheric turbulence, its spatial distribution scaling and its temporal dynamics make it possible to measure and compensate the turbulence-induced distortions in real time. The method for active dynamic aberration compensation is known as adaptive optics $(\mathrm{AO})$.

Fig. 2.1 shows the basic setup of the "conventional" adaptive optics system. Light that comes into a telescope and is distorted by the atmosphere is split into two parts. Part of the light is directed to the wave front sensor (WFS) optically conjugated to the entrance pupil, which measures the wave front excursions from the flat shape. Based on the information provided by the WFS, the correction signal is computed and sent to the second main part of AO system, the deformable mirror (DM). The DM is an electromechanical device, whose reflective surface is capable of deforming to the extent of few micrometers according to the electrical signal applied to it. When conjugated to the entrance pupil, DM introduces an aberration that can at least partially compensate the aberration introduced by the atmosphere. Since the parameters of an AO system such as spatial resolution of both the WFS and the DM necessary for effective compensation, dynamic range of turbulence-induced aberrations, and the time scale for their temporal dynamics are determined by the nature of atmospheric turbulence, a mathematical theory to describe the turbulence phenomena is necessary.

\subsection{Atmospheric turbulence theory.}

The mathematical theory capable of adequate description of turbulence effects was first introduced in the work of Soviet scientists A. N. Kolmogorov [14] and V. I. Tatarskii [15]. It has been shown that, according to the principles of fluid mechanics, the process 


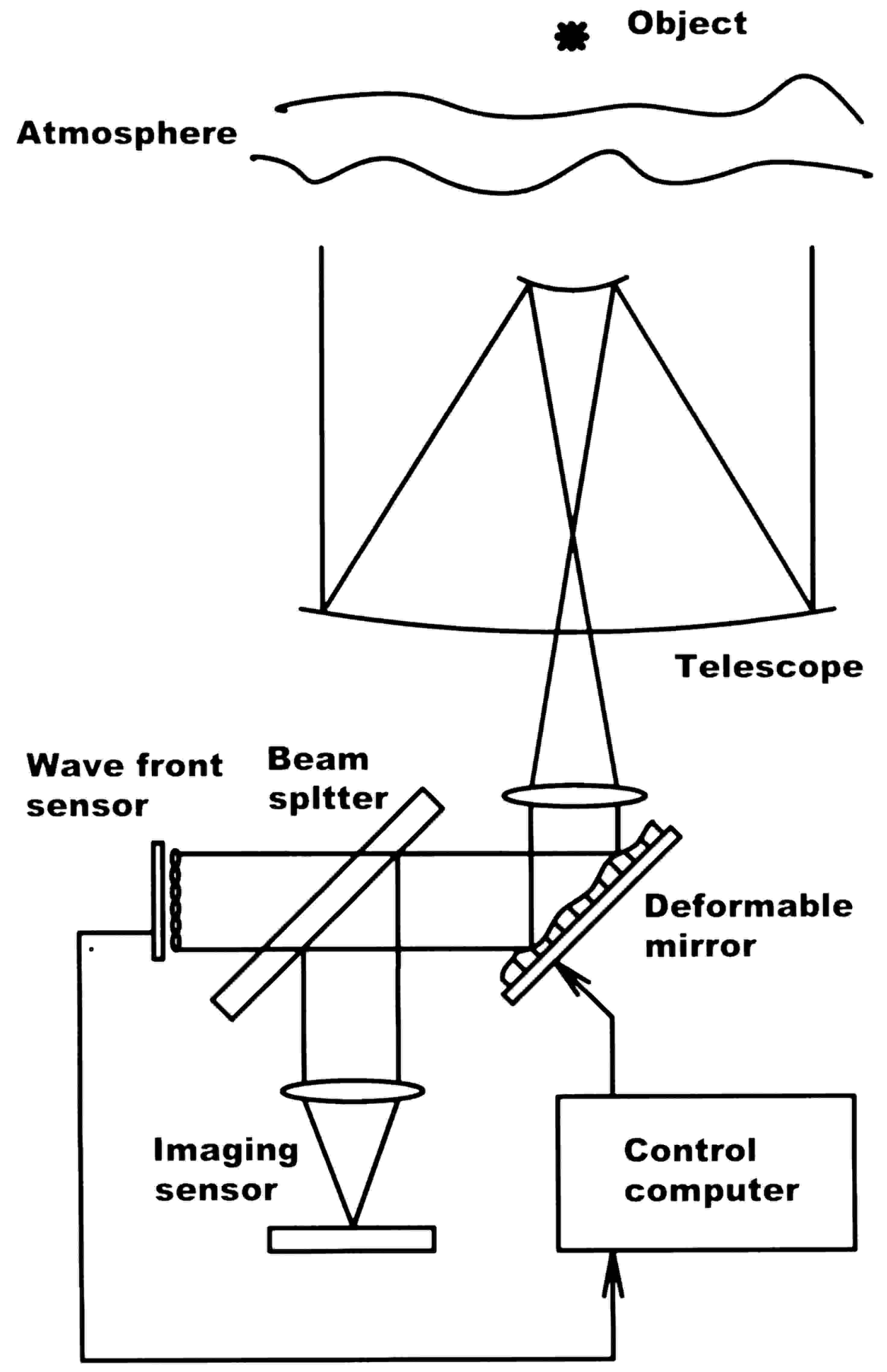

Figure 2.1: Setup of the conventional adaptive optics system. 
of energy dissipation in a volume of air results in a random density distribution of air. The random distribution of local density induce the random distribution of the refraction index of air, which is the physical property responsible for the light propagation through atmosphere. The random refractive index in the volume of air can be written as

$$
n(\vec{x}, t)=n_{0}+\delta n(\vec{x}, t)
$$

where $n_{0}$ is the mean value of the refractive index, which is very close to one, and $\delta n(\vec{x}, t)$ is the zero-mean random refractive index fluctuation responsible for atmosphereinduced aberrations. $\delta n$ is related to the local temperature $T$ and pressure $P$ by [62]

$$
\delta n=\frac{77.6 P}{T} \times 10^{-6} .
$$

At least with respect to the cumulative effect of light propagation through relatively thick layers of atmosphere (hundreds of meters), the refractive index fluctuation can be regarded as spatially Gaussian zero-mean random process. Over the periods of time as long as a few seconds the turbulence can be regarded as stationary. Therefore, its spatial statistics are completely determined by the covariance function $\Gamma(\vec{x})$ or, equivalently, by the power spectral density (PSD) $\Phi(\vec{k})$ related to each other through Fourier transform

$$
\Phi(\vec{k})=\int \Gamma(\vec{x}) \exp (-i \vec{k} \cdot \vec{x}) d \vec{x}
$$

The fundamental result of the Kolmogorov theory is the expression for PSD of the refraction index fluctuations:

$$
\Phi_{n}(\vec{k})=0.033 C_{n}^{2}|\vec{k}|^{-11 / 3}
$$

where $C_{n}^{2},\left[m^{-2 / 3}\right]$, is the structure constant of the refraction index fluctuations that determines strength of the turbulence, $\vec{k}$ is the position vector in frequency domain, also referred to as the wave number vector. Eq. (2.4) illustrates the fractal nature 
of the turbulence, which is believed to consist of "eddies", the regions of constant refractive index imbedded into one another. Eq. (2.4) is valid within so called "inertial sub-range" $k_{0} \leq|\vec{k}| \leq K_{0}$ of spatial frequencies, which is due to the fact that turbulent eddies cannot be larger that certain size $L_{0}=2 \pi / k_{0}$ called the outer scale and smaller than certain size $l_{0} 2 \pi / K_{0}$ called the inner scale. A convenient approximation to the refraction index fluctuations PSD that is valid for all spatial frequencies and takes into account both inner and outer scales

$$
\Phi_{n}(\vec{k})=0.033 C_{n}^{2}\left(|\vec{k}|^{2}+K_{0}^{2}\right)^{-11 / 6} \exp \left(-\frac{|\vec{k}|^{2}}{k_{0}^{2}}\right)
$$

is called von Karman turbulence spectrum. Another convenient turbulence characteristic, the structure function $D_{n}(\vec{x})$ of refractive index fluctuations, is defined as

$$
D_{n}(\vec{x})=\left\langle\left[\delta n\left(\vec{x}_{0}\right)-\delta n\left(\vec{x}_{0}+\vec{x}\right)\right]^{2}\right\rangle .
$$

The relation of the structure function to the turbulence autocorrelation and PSD is

$$
\begin{gathered}
D_{n}(\vec{x})=2\left[\Gamma_{n}(0)-\Gamma_{n}(\vec{x})\right] \\
=\int d|\vec{k}| \Phi_{n}(|\vec{k}|)\left[1-\frac{\sin |\vec{k}||\vec{x}|}{|\vec{k}||\vec{x}|}\right] .
\end{gathered}
$$

In the case of Kolmogorov spectrum (2.4) integration in the last expression can be done analytically yielding for the structure function

$$
D_{n}(\vec{x})=C_{n}^{2}|\vec{x}|^{2 / 3}
$$

Application of Kolmogorov theory to wave propagation through turbulent media has been developed in the works of D. L. Fried [16]. The simplified version of it convenient for AO systems analysis can be summarized as follows [62]. The basic assumptions are:

- The whole atmosphere from space to ground is divided into a number of layers with constant $C_{n}^{2}$. 
- The layers are supposed to be mutually statistically independent, which yields for the autocorrelation function of the whole atmosphere

$$
\Gamma_{n}(\vec{x})=\prod_{i=1}^{N} \Gamma_{n_{i}}(\vec{x}),
$$

where $\Gamma_{n_{i}}(\vec{x})$ is a spatial autocorrelation function of $i^{\text {th }}$ individual layer, $N$ number of layers.

- Each layer is replaced with infinitely thin two-dimensional phase screen, whose parameters are obtained by averaging over the physical thickness of a layer.

For the unit amplitude of the plane wave incident normally to the $i^{\text {th }}$ layer the transmitted optical field is

$$
u_{i}(\vec{x})=\exp \left[i \phi_{i}(\vec{x})\right]
$$

where the phase accumulated passing through $i^{\text {th }}$ atmosphere layer of thickness $\Delta z_{i}$ is

$$
\phi_{i}(\vec{x})=k \int_{z_{i}}^{z_{i}+\Delta z_{i}} d z \delta n(\vec{x}, z) .
$$

With the assumption of the turbulence being Gaussian we have for the $i^{\text {th }}$ layer phase autocorrelation function

$$
\begin{aligned}
\Gamma_{u_{i}}(\vec{x})= & \left\langle\exp \left[i \phi_{i}\left(\vec{x}_{0}\right)\right] \exp \left[i \phi_{i}\left(\vec{x}_{0}+\vec{x}\right)\right]\right\rangle \\
& =\exp \left[-\frac{1}{2} D_{\phi_{i}}(\vec{x})\right]
\end{aligned}
$$

where

$$
\begin{gathered}
D_{\phi_{i}}(\vec{x})=k^{2}\left\langle\left(\int_{z_{i}}^{z_{i}+\Delta z_{i}} d z\left[\delta n\left(\vec{x}_{0}, z\right)-\delta n\left(\vec{x}_{0}-\vec{x}, z\right)\right]\right)^{2}\right\rangle \\
=2.91 k^{2} \Delta z C_{n_{i}}^{2}|\vec{x}|^{5 / 3} .
\end{gathered}
$$

is the phase structure function of the $i^{\text {th }}$ layer. With the Fried parameter of the $i^{t h}$ layer

$$
r_{0_{i}}=0.185\left[\frac{4 \pi^{2}}{k^{2} C_{n_{i}}^{2} \Delta z_{i}}\right]^{3 / 5}
$$


introduced, the expression for the phase structure function in Eq. (2.12) takes the form

$$
D_{\phi_{i}}(\vec{x})=6.88\left(\frac{|\vec{x}|}{r_{0_{i}}}\right)^{5 / 3} .
$$

Substitution of Eq. (2.11) into Eq. (2.9) yields

$$
\begin{aligned}
\Gamma_{u}= & \exp \left[-\frac{1}{2} 6.88\left(\frac{\vec{x}}{r_{0}}\right)^{5 / 3}\right] \\
& =\exp \left[-\frac{1}{2} D_{\phi}(\vec{x})\right]
\end{aligned}
$$

where the integral Fried parameter $r_{0}$ is

$$
r_{0}=0.185\left[\frac{4 \pi^{2}}{k^{2} \sum_{i=1}^{N} C_{n_{i}}^{2} \Delta z_{i}}\right]^{3 / 5} .
$$

The Fried parameter can be interpreted as the aperture size beyond which further increase in telescope diameter results in no further increase in resolution. Quantity $\left(D / r_{0}\right)^{2}$, where $D$ is the system's entrance pupil diameter, is used as an estimate of the required number of $\mathrm{AO}$ system degrees of freedom to achieve the diffraction-limited turbulence compensation. The number of AO system degrees of freedom is normally understood as a number of WFS measurements or DM actuators.

\subsection{Model for turbulence temporal dynamics.}

In contrast to the spatial component, the time evolution of atmospheric turbulence, is not very well understood. Two processes are believed to contribute to the temporal changes in the turbulence pattern:

1. Relatively slow random motion of the eddies due to processes of energy dissipation in the air [17] sometimes referred to as "atmospheric boiling".

2. Faster deterministic motion of the atmospheric layers due to wind. Typical wind speeds measured for good atmospheric sites $[6,59]$ vary from 5 to $50 \mathrm{~m} / \mathrm{s}$ with 
typical time scale on which significant wind speed and direction changes occur of the order of ten seconds.

Rate of change in the turbulence phase patterns is an important parameter affecting the requirements for how fast an $\mathrm{AO}$ system should be to adequately follow changes in atmosphere-induced aberrations. Taylor Frozen Flow Hypothesis is the usual assumption made to model temporal dynamics. It states that the boiling effects can be neglected and all changes in the turbulence pattern are ascribed to the deterministic wind shift only. In other words, the turbulence pattern is assumed to be fixed once and for all and moving as a whole parallel to the ground with some wind speed $\vec{v}$. The measure of how fast the phase changes in the telescope aperture plain occur was first introduced in [18] and called the Greenwood frequency. Greenwood frequency, $f_{G}$, is defined as the inverse of the time lag in an AO system, for which the lag-induced phase error reaches, in the absence of any other errors, 1 rad. For a single layer the definition for $f_{G}$ is

$$
f_{G}=0.427 \frac{|\vec{v}|}{r_{0}} .
$$

For the thick atmosphere with many layers moving with different speeds the wind speed in Eq. (2.17) should be somehow averaged over altitude. The turbulence weighted wind speed average

$$
|\overline{\vec{v}}|=\left[\frac{\int d z C_{N}^{2}(z)|\vec{v}(z)|^{5 / 3}}{\int d z C_{N}^{2}(z)}\right]^{3 / 5}
$$

is normally used in Eq. (2.17), which means that the high velocities at regions where $C_{n}^{2}$ is large give the biggest contribution to the Greenwood frequency. Typical value of Greenwood frequency for good astronomical sites in near infrared is between 20 and $30 \mathrm{~Hz}$. To minimize the lag-induced fraction of the wave front compensation error the AO system closed-loop bandwidth should match or exceed the Greenwood frequency. This consideration will be seen later to govern the sampling rate of the AO controller. 


\subsection{Image quality assessment.}

In astronomical imaging objects are considered to be spatially incoherent, that is the object autocorrelation function has the form

$$
\Gamma_{o}\left(\vec{x}, \vec{x}^{\prime}\right)=o(\vec{x}) \delta\left(\vec{x}-\vec{x}^{\prime}\right)
$$

where $o(\vec{x})$ is the object irradiance function. The fundamental result for an incoherent optical system is that the spectrum of the image intensity is determined by the autocorrelation function $\Gamma_{p}(\vec{x})$ of the incident field in the pupil, and the generalized pupil function $W_{g}(\vec{x})[60]$ :

$$
\begin{gathered}
I(\vec{f})=\Gamma_{p}\left(\lambda d_{i} \vec{f}\right) \int d \vec{x} W_{g}(\vec{x}) W_{g}\left(\vec{x}-\lambda d_{i} \vec{f}\right), \\
W_{g}(\vec{x})=t_{a}(\vec{x}) W_{a}(\vec{x}), \quad \int d \vec{x} W_{a}(\vec{x})=1,
\end{gathered}
$$

where $d_{i}$ is the distance from pupil to the image plane, $t_{a}(\vec{x})$ is the pupil transmittance function and $W_{a}(\vec{x})$ is a function constant inside the pupil and zero elsewhere. If the atmosphere is modelled as a thin phase screen with transmittance function $t_{a}(\vec{x})$, the autocorrelation function of the field in the pupil is [62]

$$
\Gamma_{p}(\vec{x})=\Gamma_{a}(\vec{x}) \Gamma_{o}(\vec{x})
$$

where $\Gamma_{a}(\vec{x})$ is the field autocorrelation due to the atmosphere and $\Gamma_{o}(\vec{x})$ is the field autocorrelation due to the object. Substituting this into Eq. (2.20) gives

$$
I(\vec{f})=\Gamma_{a}\left(\lambda d_{i} \vec{f}\right) \Gamma_{o}\left(\lambda d_{i} \vec{f}\right) \int d \vec{x} W_{g}(\vec{x}) W_{g}\left(\vec{x}-\lambda d_{i} \vec{f}\right) .
$$

By the van Cittert-Zernike theorem [62], the second term here can be recognized as the scaled Fourier transform of the object irradiance function, $o(\vec{x})$, whereas the integral term is, up to a multiplicative constant, the diffraction-limited optical transfer func-

tion $H_{o}(\vec{f})$ of the imaging system for incoherent illumination [62]. Thus, the previous 
equation takes the form

$$
I(\vec{f})=O(\vec{f}) \Gamma_{a}\left(\lambda d_{i} \vec{f}\right) H_{o}(\vec{f})
$$

and the system transfer function $I(\vec{f}) / O(\vec{f})$ is

$$
H(\vec{f})=\Gamma_{a}\left(\lambda d_{i} \vec{f}\right) H_{o}(\vec{f})=H_{a}(\vec{f}) H_{o}(\vec{f})
$$

Substitution of Eq. (2.15) gives

$$
H(\vec{f})=\exp \left[-\frac{1}{2} D_{\phi}\left(\lambda d_{i} \vec{f}\right)\right] H_{o}(\vec{f})
$$

which is valid for any structure function provided that the corresponding random process is Gaussian. For the uncompensated Kolmogorov turbulence we will have

$$
H(\vec{f})=\exp \left[-\frac{1}{2} 6.88\left(\frac{\lambda d_{i}|\vec{f}|}{r_{o}}\right)^{5 / 3}\right] H_{o}(\vec{f}) .
$$

Optical transfer function (OTF) $H(\vec{f})$ is the main measure of the imaging system's performance. The absolute value of the normalized transfer function $H(\vec{f}) / H(\overrightarrow{0})$ gives the attenuation factor for a spatial harmonic of frequency $\vec{f}$ as it passes the imaging system. Transfer function equal to unity for all frequencies corresponds to the ideal imaging system. Other useful quality measures are derived from the transfer function. The inverse Fourier transform of the transfer function defines the impulse response, or point-spread function (PSF) of the system

$$
s(\vec{x})=\mathscr{F}^{-1}[H(\vec{f})],
$$

which shows the degree of the point source image blur caused by the optical system. The Strehl ratio is defined as the ratio of the center intensity of the real system PSF to the center intensity of the diffraction-limited PSF in the absence of aberrations:

$$
S R=s(\overrightarrow{0}) / s_{\text {diff }}(\overrightarrow{0})
$$


Strehl ratio gives a convenient measure of the image quality departure from the limiting quality physically achievable for the system.

Eq. (2.23) is the most important result of this section. It is obvious from this equation that, to achieve diffraction-limited imaging, it is necessary to make the exponential term equal to unity for all frequencies, which is equivalent to the structure function $D_{\phi}(\vec{x})$ being equal to zero for all separations. With the definition of the structure function given in Eq. (2.7) this means that the phase in the pupil should be just constant. Therefore, in order to improve image quality, an AO system should be able to minimize the departure of the pupil phase function from a constant value. This gives motivation for the introduction of another useful measure for the imaging system quality, the pupil averaged residual phase variation

$$
\sigma_{\phi}^{2}=\frac{1}{|S|} \int_{S} d \vec{x}\left\langle(\phi(\vec{x})-\langle\phi(\vec{x})\rangle)^{2}\right\rangle,|S|=\int_{S} d \vec{x},
$$

where $S$ is the area if the entrance pupil. Obviously, the less this value is the closer the system is to the diffraction limit. Beside the relative ease this parameter can be computed with in the $\mathrm{AO}$ system simulations, $\sigma_{\phi}^{2}$ is connected to Strehl ratio, the most important AO system quality measure, through Marechal's approximation [61]

$$
S R \approx \exp \left[-\sigma_{\phi}^{2}\right]
$$

Thus, to maximize the Strehl ratio means to minimize the pupil averaged residual phase variation, so this quantity can be taken as a merit function in the optimal AO control algorithms.

\subsection{Factors limiting AO system performance.}

In this section the main factors that prevent ideal turbulence compensation by means of adaptive optics will be listed [61]. 


\subsubsection{Anisoplanatism.}

Anisoplanatism is one of the most important effects to limit AO system performance. In contrast to the ideal situation shown on Fig. 2.1, where the light from the object to be imaged is also used for measuring the wave front errors caused by the atmosphere, it is sometimes necessary to use for wave front sensing another, brighter, object referred to as the beacon. This is because most astronomical objects of interest are extremely faint, or emit most of their radiation in a spectral range unavailable for wave front sensing. This leads to the situation depicted in Fig. 2.2, where one object is available for measuring the wave front distortion, while a different object is to be measured. Two cases shown in the figure correspond to:

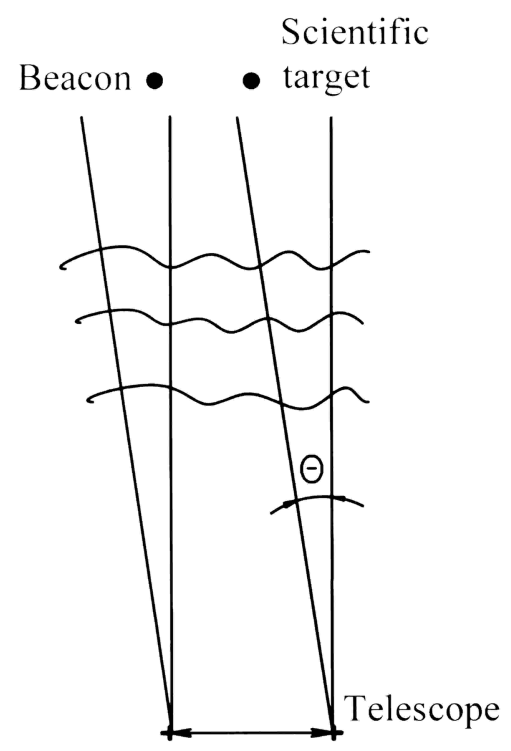

(a)

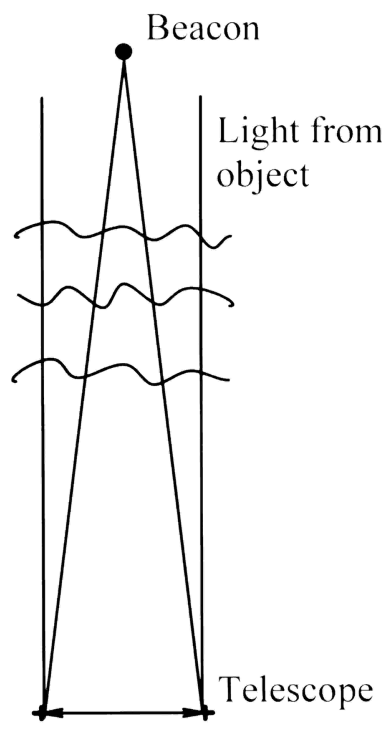

(b)

Figure 2.2: Angular (a) and focal (b) anisoplanatism.

1. The situation shown on Fig. 2.2(a) is when a bright natural star that happens to exist near the object of interest is taken for the infinitely remote beacon or 
natural guide star (NGS). Light from the beacon passes through atmosphere in the direction different from the scientific target direction by angle $\theta$.

2. The situation shown on Fig. 2.2(b) is when an artificial beacon is created based normally on the scattering of the laser light in high atmospheric layers, which is referred to as laser guide star (LGS). Because of the physical nature of such a beacon, its altitude is finite. For instance, it is $\sim 90 \mathrm{~km}$ for sodium layer LGSs. This implies that the light from an artificial beacon passes through only part of atmospheric turbulence within a conical volume swept by the rays emanating from the beacon and collected by the telescope aperture.

It is obvious from Fig. 2.2 that the problem arises because of the fact that the light from the beacon passes through a slightly different region of the atmosphere than the light from the scientific target. This is the underlying case of anisoplanatism. The anisoplanatic error in the wave front correction occurs due to mismatch of the information provided by WFS and the actual wave front distortion. The aperture averaged phase error caused by the angular anisoplanatism, that is natural guide star's being an angle $\theta$ away from scientific target, can be approximated by [61]

$$
\sigma_{\text {angular }}^{2}=\left(\frac{\theta}{\theta_{0}}\right)^{5 / 3}
$$

where the anisoplanatic angle $\theta_{0}$ is defined as a separation angle for which the phase error is $1 \mathrm{rad}^{2}$ in the AO system with no other errors. The explicit value for $\theta_{0}$ in the case of Kolmogorov turbulence is

$$
\theta_{0}=0.31\left(\frac{r_{0}}{\bar{h}}\right), \bar{h}=\left[\frac{\int d z C_{n}^{2}(z) z^{5 / 3}}{\int d z C_{n}^{2}(z)}\right]^{3 / 5} .
$$

Typical value for anisoplanatic angle computed with the aid of Eq. (2.28) does not exceed 3 arcsec even for good astronomical sites, which applies severe limitation on 
the size of AO-compensated field of view. An analogous expression holds for the focal anisoplanatism, i.e. the phase error due to laser guide star being at a finite altitude:

$$
\sigma_{\text {focal }}^{2}=\left(\frac{D}{d_{0}}\right)^{5 / 3}
$$

where parameter $d_{0}$ can be interpreted as the diameter of the aperture over which the wave front error due to the focal anisoplanatism is equal to $1 \mathrm{rad}^{2}$ in the $\mathrm{AO}$ system with no other errors [19]. $d_{0}$ depends on the guide star altitude and for sodium LGS $(H=90 \mathrm{~km})$ it varies from 2 to 4 meters, i.e. the effect of focal anisoplanatism is less restrictive than that of angular anisoplanatism.

\subsubsection{Temporal error.}

This fundamental error is caused by the inability of the AO system to respond instantly to the changes in the wave front. Measuring of the wave front error by WFS with acceptable accuracy takes time, since collecting sufficient photons for adequate SNR requires time, as well as processing the WFS data to obtain the correction command. Time delays occur in process of transferring the information among the pieces of AO system hardware (WFS-computer-DM). A simple assessment of the temporal phase error can be done if the AO system temporal behavior is modeled as the action of some kind of integrator with integration time $\triangle T$. In this case the temporal phase error can be written as [18]

$$
\sigma_{\text {temp }}^{2}=K\left(\frac{f_{G}}{\Delta f}\right)^{5 / 3}
$$

where $\triangle f=1 / \triangle T$ is the integrator bandwidth, constant $\mathrm{K}$ depends on the form of frequency response of the servo loop and takes values from 0.2 to 1 [61].

\subsubsection{Measurement error.}

The error in the measurement data provided by the WFS has two fundamental sources: 
- measurement noise;

- finite spatial sampling of wave front.

One contribution to the measurement noise is the photon noise, whose value depends on the illumination level and is described by the Poisson statistics. As the light intensity on the detector increases this noise increases proportionally to the square root of intensity so that the detected signal-to-noise ratio (SNR) increases.

The read noise typical for CCD detectors is another contribution to the measurement noise. The level of this noise is constant independently of the illumination, and can be described by the Gaussian statistics.

The SNR for a general photodetector has the form [61]

$$
S N R=\frac{n_{p}}{\left(n_{p}+n_{b}^{2}+\sigma_{r}^{2}\right)^{1 / 2}}
$$

where $n_{p}$ is the number of detected photoelectrons, $n_{b}$ is the number of the background electrons due to thermal noise, amplifier noise, scattered light, and $\sigma_{r}$ is the read noise variance that do not depend on the level of incident radiation.

The phase error due to the combined effect of measurement noise and finite sampling has the following generic form [61]

$$
\begin{gathered}
\sigma_{\text {meas }}^{2}=K\left(\frac{d / r_{0}}{S N R}\right)^{2}, d>r_{0}, \\
\sigma_{\text {meas }}^{2}=K\left(\frac{1}{S N R}\right)^{2}, d<r_{0},
\end{gathered}
$$

where $d$ is the size of WFS subaperture, $\mathrm{K}$ the proportionality constant characterizing the detector's quality. 


\subsubsection{DM fitting error.}

A deformable mirror is a device for making phase correction. However, it is not perfect. The fundamental reason for its imperfection is finite spatial resolution due to the finite distance between actuators. As a result, the DM cannot exactly fit the incident wave front, which is the underlying cause of DM fitting error. Its value depends mainly on the statistics of the wave fronts to fit and the distance $d$ between DM actuators. For Kolmogorov PSD (2.4) we will have [61]

$$
\sigma_{f i t}^{2}=\mu\left(\frac{d}{r_{0}}\right)^{5 / 3},
$$

where $\mu$ is the constant, $0.1 \leq \mu \leq 0.3$. The exact value for $\mu$ depends upon the characteristics of the deformable mirror, such as mirror type (segmented piston or piston-tilt, continuous face-sheet), and the actuator arrangement (square, triangular or hexagonal). It is important to note that, in the case of both measurement and fitting error, choosing the inter-actuator distance or subaperture size less than $r_{0}$ will improve wave front compensation performance.

\subsubsection{Other sources of errors.}

There are also a number of error sources due to imperfections in the technology involved in making $\mathrm{AO}$ system elements. The most important of these are:

- Nonlinearities in the DM and WFS output, and the limited dynamic range of both the DM and WFS, which may cause errors due to saturation.

- Manufacturing defects such as irregularities in DM actuator or WFS subaperture positions, aberrations and geometrical misalignments of optical elements, which may not only deteriorate the system performance but also cause its instability. 
- Different optical paths to the WFS, DM, and to the imaging camera result in so called non-common path errors, which can also be significant.

- Scattered light in the system, which causes background illumination of the sensor and thus reduces the SNR according to the Eq. (2.31).

- The additional error results from the "model uncertainties" due to simplifying assumptions made for the underlying physical processes which are difficult or impossible to model exactly. Layered atmosphere model, Frozen Flow Hypothesis or linearity of AO system elements are such assumptions usually made, to mention just a few.

All the errors mentioned must be somehow put together to estimate the overall error of the AO system. This will make an $\mathrm{AO}$ system error budget. With the assumption that the sources of each error are independent random processes, the resulting phase error variance is just the sum of variances due to the individual errors. According to the discussed above this sum looks like [61]

$$
\sigma^{2}=\sigma_{\text {aniso }}^{2}+\sigma_{\text {fit }}^{2}+\sigma_{\text {temp }}^{2}+\sigma_{\text {meas }}^{2}+\sigma_{\text {recons }}^{2}+\sigma_{\text {tech }}^{2}
$$

Since the assumption of error independence is not true for real systems, the previous equation overestimates the overall error. Desire to find more accurate overall error estimate provides motivation for doing the AO system simulations. Nevertheless, it is reasonable to assume that significantly bigger (smaller) errors make the correspondingly big (small) contribution. Because of the many possibly correlated error factors, it is not necessarily wise to make any particular one very small. Rather, these errors need to be balanced. 


\subsection{Multi Conjugate AO.}

Multi-conjugate adaptive optics (MCAO) is a promising approach to increase the turbulence-corrected field of view (FoV). As it was pointed out in Sec. 2.5.1 that in the case of a single beacon the compensated FoV is strongly limited by the angular anisoplanatism, and is generally less than 3 arcsec. This makes it impossible to observe with high resolution such large astronomical objects as galaxies or the planets of our Solar System, which normally requires FoV of the order of 1 arcmin. MCAO can, in principle, solve this problem. The performance improvement is achieved by means of probing the atmospheric turbulence in multiple directions, as shown on Fig. 2.3, with multiple natural and laser guide stars, which is referred to as "star-oriented" MCAO, or probing the turbulence in a number of layers, such as "layer-oriented" approach advocated by Ragazzoni et. al. [22, 23]. The extra information obtained is used to correct the turbulence not only in aperture plane, but also in the volume of atmosphere with the aid of multiple deformable mirrors (DMs) conjugated to different altitudes.

The ability of a multiple-DM system to correct atmospheric turbulence in a wider range of directions arises by the virtue of the fact that DMs conjugated to different altitudes affect the aberration compensation different ways. First proposed in 1988 [20], the MCAO concept has become an object of extensive research. Its increasing importance accounts for the fact that MCAO is supposed to be the key technology for the new generation of extremely large telescopes.

From a mathematical standpoint the process of turbulence correction in MCAO can be considered a form of tomography: the wave front sensors can only measure the cumulative effect of turbulence in the aperture plane in a number of different directions. The set of measured data that consists of information about the different, but overlapping volumes of atmosphere, needs to be processed to extract the 3D structure of turbulence on the way of light collected by the telescope. The commands for the 


$$
0^{\text {NGS1 }} \quad 0^{\text {NGS2 }}
$$

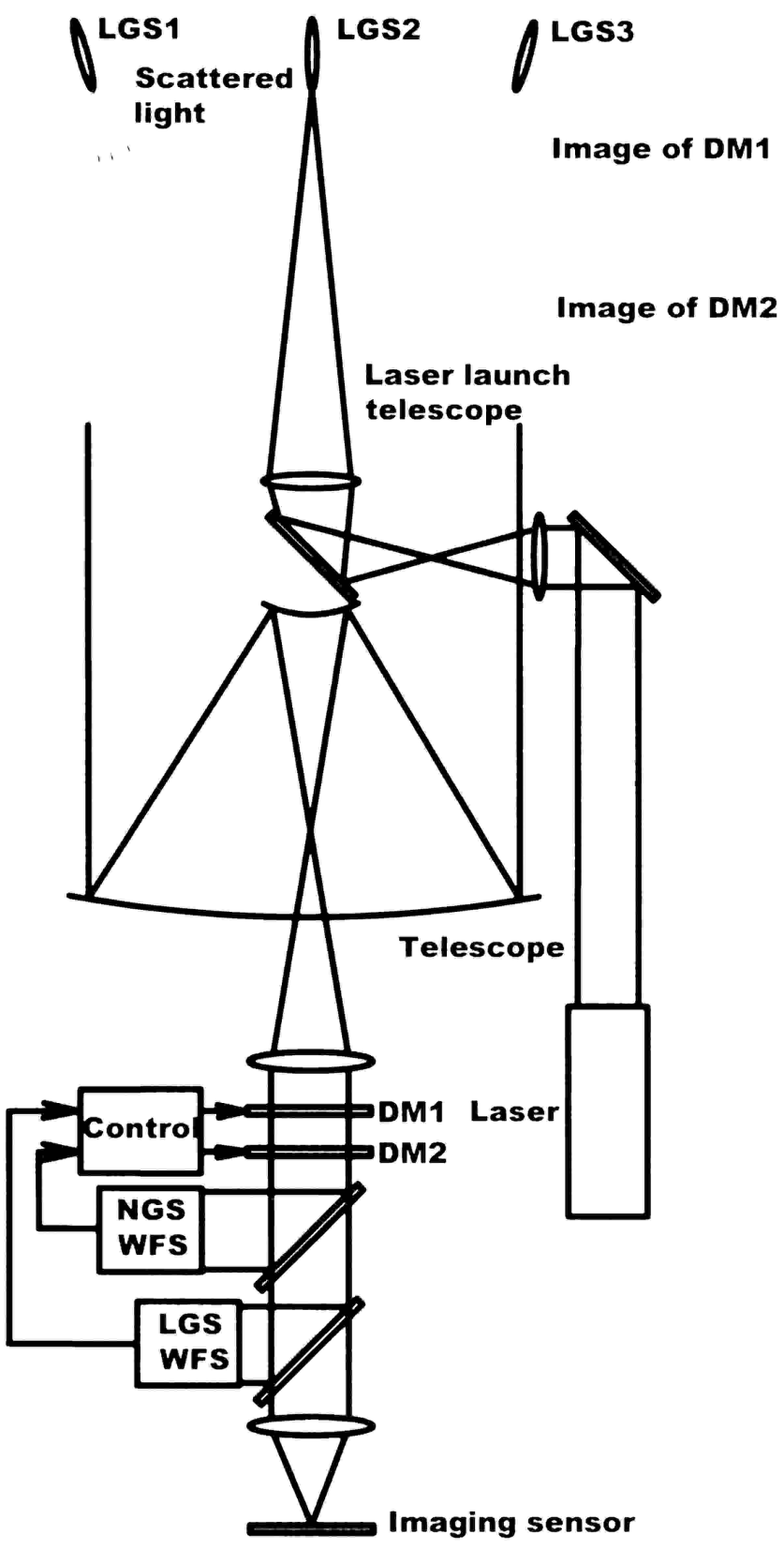

Figure 2.3: Multi Conjugate Adaptive Optics system layout. 
DMs are computed in a way to minimize the difference between the 3D tomographic phase estimate and DM correction projected onto the telescope aperture plane and averaged over a number of viewing directions. Similar to most tomographic problems, the problem of MCAO control is ill-posed owing to the obvious fact that limited resolution WFSs probing the turbulence in a limited number of directions produce only a limited amount of information, which is insufficient to extract the full 3D turbulence profile. Another complication arises from additive nature of both turbulence and the action of DMs on the overall residual phase, which results in a multiplicity of turbulence and DM correction phase configurations producing the same cumulative effect in the main aperture, and hence non-uniqueness of the MCAO control problem solution. The significantly higher hardware complexity of the problem dictated by the very idea of MCAO is one more complication, which presents a non-trivial problem of finding the numerically effective algorithms.

Theoretical analysis of the MCAO systems is more complicated than analyzing "conventional", or single conjugate AO (SCAO). In a series of papers $[24,25,26]$ A. Tokovinin et. al. gave theoretical estimates of the performance gain achievable with MCAO systems along with some recommendations about the optimal system configurations. With simplifying assumptions of infinite aperture, infinite resolution noiseless WFS measurement, perfect DM correction and Kolmogorov turbulence the following facts about MCAO system performance are derived:

- The scaling law for residual phase error of MCAO system is formally the same as for the SCAO one:

$$
\left\langle\sigma(\vec{\theta})^{2}\right\rangle=e_{K}(\vec{\theta})\left(\frac{\Theta}{\theta_{K}}\right)^{5 / 3},
$$

where $K$ is the number of guide stars, $e_{K}(\vec{\theta})$ is a scaling function depending on the observation direction and guide stars geometry, $\Theta$ is the angular size of guide stars constellation and $\theta_{K}$ is the generalized isoplanatic angle that depends on 
the number of guide stars and their configuration.

- The achievable by MCAO increase of compensated field of view, that is the increase in $\theta_{K}$ in comparison to $\theta_{0}$ (see Eq. (2.28)) is expected to be about tenfold for $\lambda=1 \mu \mathrm{m}$, which makes a leap from $6 \operatorname{arcsec}$ FoV to 1 arcmin.

- If only $M$ deformable mirrors are used, there exists a set of $M$ optimal altitudes for them, which can be found by minimizing some merit function depending on $C_{n}^{2}$ profile [24].

- In order to achieve better correction for wider FoV it is always necessary to trade off the image quality in a single point and the one averaged over entire FoV, which leads to the conclusion that MCAO systems are not able to produce Strehl ratios significantly larger than 0.5 homogeneously over wide range of directions.

- The performance estimates made under the infinite aperture assumption with the aid of Fourier methods, are the more accurate the larger real aperture diameter $D$ is and become quite accurate for the giant telescopes with $D>30 \mathrm{~m}$. This observation led to the extensive research in the application of Fourier methods for giant telescopes AO [27, 28, 29] very well summarized in B. Ellerbroek's treatise [30].

Despite the possibility to evaluate the MCAO system performance under a number of simplifying assumptions, the performance of real systems may differ from its theoretical estimates because of additional factors that are difficult or impossible to take into account analytically. As it was pointed out in Ref. [24], the numbers obtained there are neither under- nor overestimates of the performance of real system, but rather just approximations, and real systems may very well show better performance than the "ideal" ones. The next step on the way from theoretical analysis to the real AO systems installed on the new telescopes is the performance evaluation using more accurate 
and detailed mathematical models both for $\mathrm{AO}$ system elements and for atmospheric turbulence. That is why the numerical simulations of MCAO systems are desirable and constitute a significant fraction of the research in the area.

The results presented in this dissertation are concerned mostly with the numerical analysis of multi conjugate adaptive optics systems together with the analysis of possible control algorithms for them. Next chapter summarizes the approaches used for mathematical modeling of the large scale MCAO systems. 


\section{CHAPTER 3}

\section{Mathematical modeling of AO systems.}

In this chapter a method of simulating an $\mathrm{AO}$ system, and mathematical models for its elements and their interconnections are described. All the results reported in the next chapters are based on the equations, and are subject to the simplifying assumptions listed in this chapter. For the sake of brevity only the model for MCAO system is described below. The model for SCAO system can be viewed as a simplified special case.

\subsection{Model for atmosphere.}

The following assumptions are used to model atmospheric turbulence effects on optical systems.

1. Layered model is employed to model the 3D atmospheric turbulence, that is the turbulence is assumed to be concentrated in a finite number of infinitely thin, statistically independent phase screens, whose statistical properties are found by applying the discretization procedure to the continuous turbulence profile $\left(C_{n}^{2}\right.$ and wind speed) measured at the astronomical site. The simple standard discretization procedure consists of the following steps.

1. The atmosphere is divided in a number of $N_{E L}$ equally spaced slabs. 
2. The equivalent altitudes are computed according to

$$
h_{e q}^{i}=\frac{\int_{h_{i-1}}^{h_{i}} h C_{n}^{2}(h) d h}{\int_{h_{i-1}}^{h_{i}} C_{n}^{2}(h) d h}, i=1, \ldots, N_{E L},
$$

where $h_{i}$ are altitudes of layer slabs' borders.

3. Equivalent strengths $\left(C_{n}^{2}\right)^{e q}$ are found from

$$
\left(C_{n}^{2}\right)_{i}^{e q}=\frac{1}{\triangle h_{i}} \int_{h_{i-1}}^{h_{i}} C_{n}^{2}(h) d h, i=1, \ldots, N_{E L},
$$

where $\Delta h_{i}$ are thicknesses of the slabs. Correspondingly, the relative weights of layers are equal to

$$
W_{i}=\frac{\int_{h_{i-1}}^{h_{i}} C_{n}^{2}(h) d h}{\int_{h_{0}}^{h_{\max }} C_{n}^{2}(h) d h}, \sum_{i=1}^{N_{E L}} W_{i}=1
$$

4. Equivalent wind velocities $\left|\vec{v}_{i}^{e q}\right|$ are computed such that to maintain the Greenwood frequency (see Eqs. (2.17), (2.18)) the same both for 'real' and equivalent wind profiles, which results

$$
\left|\vec{v}_{i}^{e q}\right|=\left[\frac{\int_{h_{i-1}}^{h_{i}} C_{n}^{2}(h)|\vec{v}(h)|^{5 / 3} d h}{\int_{h_{i-1}}^{h_{i}} C_{n}^{2}(h) d h}\right]^{3 / 5} .
$$

Figure (3.1) shows as an example the initial measured 54-layer Cerro-Pachon turbulence profile [6] and equivalent 6-layer turbulence profile thus obtained. Simulations performed by the author to check the validity the layered atmosphere model for MCAO systems analysis show that the system performance metrics obtained with the more than 6-layer atmosphere models are virtually the same as those obtained with the quasi-continuous (tens of layers) atmosphere.

2. Frozen Flow Hypothesis (see Sec. 2.3) is assumed, that is the turbulence dynamics are determined only by the phase screens' shift caused by the wind. Absolute values of wind velocities are computed by Eq. (3.4), wind directions are supposed to be known, e.g. from measurements. 

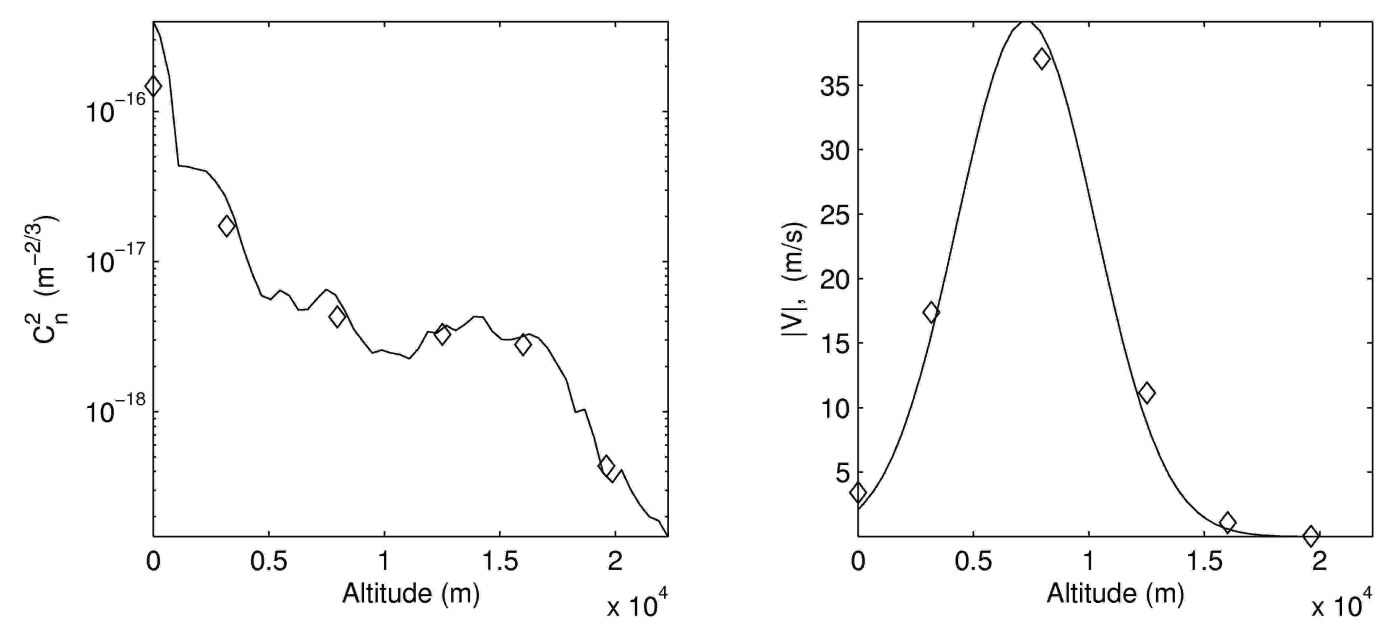

Figure 3.1: Cerro Pachon turbulence profile and its 6-layer discretization (shown with diamonds). Left panel: $C_{n}^{2}$ profile. Right panel: wind velocity profile.

3. The continuous spatial phase distributions are discretized by approximating with a finite subset of some basis in the metric of the Hilbert space $\mathscr{H}_{2}$ of functions defined on two-dimensional region $S$ on each phase screen, i.e.

$$
\phi(\vec{x}) \approx \sum_{i=1}^{N} \phi_{i} f_{i}(\vec{x}), \vec{x} \in S,
$$

where

$$
\begin{gathered}
\phi_{i}=\left(\phi(\vec{x}), \tilde{f}_{i}(\vec{x})\right), \\
(a(\vec{x}), b(\vec{x}))=\frac{1}{|S|} \int_{S} a(\vec{x}) b(\vec{x}) d \vec{x},|S|=\int_{S} d \vec{x}
\end{gathered}
$$

is a metric of $\mathscr{H}_{2}(S)$ and $\left\{\tilde{f}_{i}\right\}_{i=1}^{\infty},\left(f_{i}(\vec{x}), \tilde{f}_{j}(\vec{x})\right)=\delta_{i j}$, is the set of functions biorthogonal to $f_{i}$. Basis functions $f_{i}(\vec{x})$ used in adaptive optics are usually separated into two types:

- Localized functions, whose support is much smaller than the region $S$ but $\cup_{i=1}^{N} \operatorname{supp}\left(f_{i}\right) \supset S$ (splines, wavelets). Because of the local nature of this functions each of them influences only a small region within $S$, so such a discretization is regarded as "zonal" approach. 
- Functions for which $\operatorname{supp}\left(f_{i}\right) \supset S, \forall i$ (Zernike polynomials, trigonometric functions). Each of such functions influences the whole phase distribution, and the corresponding discretization approach is called the "global" one.

In any case the approximation of the form (3.5) results in replacement of functions with vectors of their approximation coefficients and linear operators acting on the functions with matrices, respectively. The choice of basis functions proves to be crucial for both the subsequent modelling and especially for the wave front reconstruction strategy. All results reported below are obtained for the zonal turbulence discretization approach with the bilinear splines defined on the square grid $\left\{\vec{x}_{i}\left(x_{i}, y_{i}\right)\right\}_{i=1}^{N}$ with extent $D$ and grid spacing $\Delta=D^{2} / N$

$$
f_{i}(x, y)=\left\{\begin{array}{l}
\left(1-\frac{x-x_{i}}{\Delta}\right)\left(1-\frac{y-y_{i}}{\Delta}\right),\left|x-x_{i}\right| \leq \Delta,\left|y-y_{i}\right| \leq \Delta \\
0, \text { otherwise }
\end{array}\right.
$$

Bilinear splines have a number of properties, which make these functions convenient for numerical applications:

- In contrast to Zernike polynomials, bilinear splines form the topological basis in $\mathscr{H}_{2}$, i.e. the problem of finding the approximation coefficients is stable for any $N$. The aperture averaged error of bilinear spline approximation of random phase functions with Kolmogorov statistics is [31]

$$
\sigma^{2}(\Delta)=0.28\left(\frac{\Delta}{r_{0}}\right)^{5 / 3}
$$

i.e. it quickly goes to zero as $\Delta$ decreases, which is a favorable behavior in comparison to the Zernike polynomials approximation, whose error begins to reduce very slowly for large $N[62]$.

- The coefficients $\phi_{i}$ in the case of bilinear splines are equal just to the values of 
phase $\phi(\vec{x})$ at the grid points $\left(x_{i}, y_{i}\right)$, i.e. in this case Eq. (3.5) takes the form

$$
\phi(\vec{x}) \approx \sum_{i=1}^{N} \phi\left(\vec{x}_{i}\right) f_{i}(\vec{x}),
$$

so the integration by Eq. (3.6) is not necessary. We will use this result routinely throughout the text.

- Bilinear splines $f_{i}(\vec{x})$ are nonzero only in the square region with side $2 \Delta$ around their centers $\vec{x}_{i}$, i.e. they are very localized. As it will be shown below, the use of localized spline functions leads to the effective low complexity sparse matrix formulation of the $\mathrm{AO}$ control.

4. Geometrical optics propagation through atmosphere is assumed. This means that the light propagates along straight paths so that the resulting phase perturbation in the aperture plane is obtained by summing up phase values on each individual screen projected along the straight rays emanating from each guide star. Only phase effects of turbulence are taken into account, which is justified for weak turbulence on good astronomical sites. Intensity fluctuations (scintillation) are neglected. With bilinear spline approximation applied, the action of $k^{\text {th }}$ phase screen projected on the main aperture along the $l^{\text {th }}$ guide star or scientific target is

$$
\phi_{l, k}\left(\vec{x}_{a}\right)=\sum_{i=1}^{N_{l}} \phi_{k, i} f_{l k, i}\left(\vec{x}_{a}\right),
$$

where

- $\left\{\phi_{k, i}\right\}$ are $k^{t h}$ screen phase approximation coefficients,

- $N_{l}$ is the number of approximating splines in $l^{\text {th }}$ phase screen,

- the law of the $k^{t h}$ phase screen coordinates $\vec{x}_{k}$ projection onto aperture coordinates $\vec{x}_{a}$ is

$$
\vec{x}_{a}=\frac{1}{1-h_{l}^{s t} / h_{k}^{p s}}\left(\vec{x}_{k}-h_{k}^{p s} \vec{\theta}_{l}\right)
$$


- $\left\{f_{l k, i}\right\}_{i=1}^{N}$ is the set of bilinear splines defined on the $k^{t h}$ phase grid and projected onto the aperture along $l^{\text {th }}$ guide star,

- $\theta_{l}, h_{l}^{s t}$ are the $l^{\text {th }}$ guide star (scientific target) direction angle and altitude, respectively,

- $h_{k}^{p s}$ is the $k^{t h}$ phase screen altitude.

The important characteristic of the turbulence that needs to be specified is the covariance matrix $C_{\phi \phi}=\left\langle\vec{\phi} \vec{\phi}^{T}\right\rangle$ of the phase approximation coefficients vector $\vec{\phi}$. In the framework of the layered atmosphere model with statistically independent phase screens $C_{\phi \phi}$-matrix has the block-diagonal structure, where each block $C_{\phi \phi_{k}}$ corresponds to a $k^{\text {th }}$ layer. It should be noted that, in the case of Kolmogorov turbulence, the zero frequency component, so called "piston", is infinite, which results in infinite elements of matrix $C_{\phi \phi_{k}}$. In order to obtain the finite phase covariance matrix, the piston component should be removed from the phase distribution $\phi(\vec{x})$. It can be done in two ways:

- Phase average subtraction (piston removal). The piston or DC component of the phase $\phi(\vec{x})$ defined on the region $S$ is its average $\bar{\phi}(\vec{x})$ over this region:

$$
\bar{\phi}(\vec{x})=\frac{1}{|S|} \int_{S} \phi(\vec{x}) d \vec{x},|S|=\int_{S} d \vec{x} .
$$

Let bilinear splines are considered as the basis set $\left\{f_{i}(\vec{x})\right\}_{i=1}^{N}$ for phase discretization and $S=\cup_{i=1}^{N} \operatorname{supp} f_{i}(\vec{x})$, i.e. the union of all spline supports. Then $|S| \rightarrow N \Delta^{2}$ as $N \rightarrow \infty$. Substituting the bilinear spline phase approximation (3.9) and Eq. (3.7) into Eq. (3.12), and performing integration we get

$$
\bar{\phi}(\vec{x})=\frac{1}{N \Delta^{2}} \int_{S} \phi(\vec{x}) d \vec{x} \approx \frac{1}{N} \sum_{i=1}^{N} \vec{\phi}_{i} .
$$


Thus, we can write for the bilinear approximation coefficients $\tilde{\vec{\phi}}$ of the pistonremoved phase

$$
\tilde{\vec{\phi}}=\vec{\phi}-\frac{1}{N} \sum_{i=1}^{N} \vec{\phi}_{i}=P_{p s} \vec{\phi},
$$

where the piston removal projector matrix $P_{p s}$ is defined as

$$
P_{p s}=I-\frac{1}{N}\left[\begin{array}{c}
1 \\
1 \\
\vdots \\
1
\end{array}\right]\left[\begin{array}{llll}
1 & 1 & \ldots & 1
\end{array}\right] \text {. }
$$

Since, according to Eq. (3.9), the bilinear spline approximation coefficients $\vec{\phi}_{i}$ are equal to the phase values $\phi\left(\vec{x}_{i}\right)$ in the grid points, the piston-removed phase covariance matrix for Kolmogorov turbulence takes the form [52]

$$
\begin{gathered}
\left(\tilde{C}_{\phi \phi_{k}}\right)_{i j}=\left\langle\left[\phi\left(\vec{x}_{i}\right)-\frac{1}{N} \sum_{s=1}^{N} \phi\left(\vec{x}_{s}\right)\right]\left[\left(\phi\left(\vec{x}_{j}\right)-\frac{1}{N} \sum_{t=1}^{N} \phi\left(\vec{x}_{t}\right)\right]^{*}\right\rangle\right. \\
=-\frac{1}{2}\left[D_{k}\left(\vec{x}_{i}-\vec{x}_{j}\right)-\frac{1}{N} \sum_{s=1}^{N} D_{k}\left(\vec{x}_{i}-\vec{x}_{s}\right)-\frac{1}{N} \sum_{s=1}^{N} D_{k}\left(\vec{x}_{s}-\vec{x}_{j}\right)+\frac{1}{N^{2}} \sum_{s=1}^{N} \sum_{t=1}^{N} D_{k}\left(\vec{x}_{s}-\vec{x}_{t}\right)\right],
\end{gathered}
$$

where $D_{k}(\vec{x}-\vec{y})=6.88\left(\frac{|\vec{x}-\vec{y}|}{r_{0_{k}}}\right)^{5 / 3}$ is the Kolmogorov structure function of $k^{t h}$ layer. With the structure matrix

$$
\left(D_{k}\right)_{i j}=D_{k}\left(\vec{x}_{i}-\vec{x}_{j}\right)
$$

introduced, the Eq. (3.16) can be recognized to have a simple form

$$
\tilde{C}_{\phi_{k}}=-\frac{1}{2} P_{p s} D_{k} P_{p s}^{T}
$$

which is convenient to compute also the piston-removed von Karman phase covariance matrix

$$
\tilde{C}_{\phi_{k}}=P_{p s} C_{\phi_{k}} P_{p s}^{T}
$$


where the full von Karman phase covariance matrix is [62]

$$
C_{\phi \phi_{k}}=3.089 r_{0_{k}}^{-5 / 3} \frac{\left(L_{0} / 4 \pi\right)^{5 / 6} K_{5 / 6}\left[2 \pi \rho_{i j} / L_{0}\right] \rho_{i j}^{5 / 6}}{\Gamma(11 / 6)}, \rho_{i j}=\left|\vec{x}_{i}-\vec{x}_{j}\right|,
$$

$K_{5 / 6}[\cdot]$ is the modified Bessel function of the second kind of order $5 / 6, \Gamma[\cdot]$ is the gamma function, $L_{0}$ is the von Karman turbulence outer scale.

- Phase referencing or "point removal". Since the relative phase difference for any pair of points is always finite, it is possible to regard the phase distribution as a set of phase differences with respect to the phase at some fixed reference point. This gives for "point-removed" bilinear spline approximation coefficients vector:

$$
\tilde{\vec{\phi}}=\vec{\phi}-\vec{\phi}_{r e f}=P_{p t} \vec{\phi}
$$

where $P_{p t}$ is the point removal projector matrix

$$
P_{p t}=I-\left[\begin{array}{c}
1 \\
1 \\
\vdots \\
1
\end{array}\right]\left[\begin{array}{lllllll}
0 & \ldots & 0 & 1 & 0 & \ldots & 0
\end{array}\right] \text {, }
$$

where 1 stands on the position corresponding to the reference point. This is another, equivalent, way to extract the finite part from the turbulence-induced phase error. The expressions for the point-removed phase covariance matrices for Kolmogorov and von Karman turbulence are the same as Eqs. (3.17) and (3.18) with $P_{p s}$ replaced with $P_{p t}$.

Note that piston- or point-removed phase covariance matrices are positive semi-definite and have rank deficiency 1 . 


\subsection{Wave front sensor model.}

In what follows we assume the star-oriented approach, i.e. the turbulence probing in the directions of several guide stars (see Fig. 2.3) with a WFS assigned to each guide star and conjugated to the main aperture. The equation relating WFS measurement vector $\vec{s}$ to the phase vector $\vec{\phi}$ independently of the WFS type is assumed to be linear time invariant in the form

$$
\vec{s}=G_{\phi} \vec{\phi}+\vec{n},
$$

where

- vector $\vec{s}$ is a concatenation of the wave front $x$ - and $y$-slope sub-vectors read out from each WFS,

- vector $\vec{\phi}$ is a concatenation of phase approximation coefficient sub-vectors of each turbulence layer,

- $G_{\phi}$ is the phase-to-WFS interaction matrix, one of the most important parts of the AO system mathematical model. $G_{\phi}$-matrix is computed in a way that the individual WFS measurement is modelled as the gradient of the aperture plane phase averaged over the subaperture,

- $\vec{n}$ is the concatenation vector of WFS $x$ - and $y$-slope noise components assumed to be zero mean Gaussian temporally white, uncorrelated with vector $\vec{\phi}$ and with one another.

It is important to notice that the WFS does not measure the wave front phase itself, but rather its spatial gradient and, therefore, is insensitive to piston. With $N_{g s}$ guide stars used in the system we can use Eq. (3.10) to write for the readout from a single 
WFS subaperture $W_{p}$ illuminated by the light that passed through $N_{s c r}$ phase screens

$$
\begin{gathered}
\vec{s}_{l, p}=\left[\begin{array}{c}
\vec{s}_{x} \\
\vec{s}_{y}
\end{array}\right]_{l, p}=\sum_{k=1}^{N_{s c r}} \sum_{i=1}^{N} \phi_{k, i} \frac{1}{\left|W_{p}\right|} \int_{W_{p}} \nabla f_{l k, i}\left(\vec{x}_{l k}\right) d \vec{x}_{l k}, l=1, \ldots, N_{g s}, \\
\left|W_{p}\right|=\int_{W_{p}} d \vec{x}_{l k},
\end{gathered}
$$

from where the expression for a $G_{\phi}$-matrix element in Eq. (3.21) takes the form

$$
\left(G_{\phi}\right)_{l k, p i}=\left[\begin{array}{c}
G_{\phi, x} \\
G_{\phi, y}
\end{array}\right]_{l k, p i}=\frac{1}{\left|W_{p}\right|} \int_{W_{p}} \nabla f_{l k, i}\left(\vec{x}_{l k}\right) d \vec{x}_{l k}
$$

where aperture coordinates $\vec{x}_{l k}$ are the $k^{\text {th }}$ phase screen coordinates projected along $l^{\text {th }}$ guide star according to Eq. (3.11). It is seen from Eq. (3.23) that phase-to-WFS interaction matrix of MCAO system has $N_{g s} \times N_{s c r}$ blocks and a $(l, k)$-block corresponds to the WFS measurement sub-vector regarded as an action of the $k^{\text {th }}$ phase screen on the $l^{\text {th }}$ WFS.

The theoretical importance of the phase-to-WFS interaction matrix is that it quantifies the quality of phase measurement. The singular values of $G_{\phi}$ are a measure of the turbulence-induced phase coupling to the WFS measurement. Some of the singular values being small or zero means that there exist phase configurations that are not or only weakly sensed by WFS. These configurations are given by the singular vectors of $G_{\phi}$-matrix and called the unobservable modes of the MCAO system. The fraction of turbulence projected on the unobservable modes cannot be detected by the WFS and, therefore, compensated. This puts the lower limit to the quality of turbulence compensation. Thus the WFS configuration should be chosen by a designer in a way to minimize the null space of the $G_{\phi}$-matrix.

In order to complete the measurement model, the covariance matrix $C_{n n}$ of the WFS noise needs to be specified. It is a reasonable assumption that the noise is uncorrelated between different subapertures and also between $x$ - and $y$-slope readings from a 
single subaperture. This results in the block-diagonal covariance matrix of WFS noise $\vec{n}$, where the blocks are collected in pairs, each corresponding to the $\mathrm{x}$ - and $\mathrm{y}$-slopes covariance sub-matrices, which themselves are diagonal. Their elements are equal to noise variances that can be computed by Eq. (2.32) with the proportionality factor that depends on the amount of light falling on each subaperture.

The use of laser guide stars presents an additional difficulty because of the uncertainty in the angular position of the LGS spot in the sky due to atmospheric turbulence [61]. The random movement of LGS spot around its average angular position results in random average slope, or global tilt, of the phase distribution on the main aperture the WFS is conjugated to. This makes impossible to extract the information about the global tilt from measurements with the use of a LGS. Because of the differentiating nature of the WFS measurement, the tilt in the phase transforms into constant in the measurement. This constant part of the measurement can be removed the same way as it is done for the piston in the turbulence phase, which results in piston- or point-removed noise covariance matrix:

$$
\tilde{C}_{n n}=\left[\begin{array}{cc}
P\left\langle s_{x} s_{x}^{T}\right\rangle P^{T} & 0 \\
0 & P\left\langle s_{y} s_{y}^{T}\right\rangle P^{T}
\end{array}\right],
$$

where $P$ stands for $P_{p s}$ or $P_{p t}$ and $\left\langle s_{x} s_{x}^{T}\right\rangle,\left\langle s_{y} s_{y}^{T}\right\rangle$ are diagonal matrices. Note that piston removal makes the WFS noise spatially correlated. The main problem, however, is that LGS is unable to provide global tilt information and additional NGSs for global tilt sensing are thus required. Fortunately, the anisoplanatic angle for just tilt component of the turbulence phase error is much larger than the one for the whole turbulence so it is easier to find the natural beacon for sensing just the tilt [61]. 


\subsection{Deformable mirror model.}

A linear time-invariant model is assumed for the deformable mirrors. In analogy with the atmospheric phase screens deformable mirrors are regarded as the infinitely thin phase transparencies placed at the DM conjugation planes in the atmosphere. The action of the $m^{\text {th }}$ DM projected on the main aperture along the $s^{\text {th }}$ scientific direction (direction on $s^{t h}$ scientific target) is the linear combination of the commands applied to the actuators

$$
\phi_{s, m}\left(\vec{x}_{a}\right)=\sum_{i=1}^{M_{m}} a_{m, i} r_{m, i}\left(\vec{x}-\vec{\theta}_{s} h_{m}^{d m}\right)
$$

where

- $\left\{a_{m, i}\right\}_{i=1}^{N}$ are $m^{t h} \mathrm{DM}$ actuator commands,

- $M_{m}$ is the number of actuators in $m^{\text {th }} \mathrm{DM}$,

- $\left\{r_{m, i}\right\}_{i=1}^{N}$ are the $m^{t h} \mathrm{DM}$ influence functions,

- $\vec{x}$ and $\vec{x}_{a}$ are the coordinates in $m^{\text {th }}$ DM and main aperture planes, respectively,

- $\vec{\theta}_{s}, h_{m}^{d m}$ are the $s^{t h}$ scientific direction, and $m^{t h}$ DM conjugation altitude, respectively.

The shape of the DM's influence functions $r(\vec{x})$ depends on the DM design but generally resembles an inverted bell with a small area of localization [55]. The influence functions are similar in many ways to the localized spline functions and are often approximated by splines.

The DM-to-WFS interaction matrix $G_{a}$, another key element of the AO system mathematical model, describes the relationship between the command applied to DMs and the measurement of the phase created by this command and read out from WFSs 
in the form

$$
\vec{s}=G_{a} \vec{a},
$$

where $\vec{s}$ and $\vec{a}$ are the concatenations of all WFS measurement and DM command sub-vectors, respectively. $G_{a}$-matrix is defined the same way as the $G_{\phi}$-matrix through Eq. (3.23) with obvious replacements of $f_{l k, i}\left(\vec{x}_{l k}\right)$ with $r_{m, i}\left(\vec{x}_{m, i}-\vec{\theta}_{s} H_{m}\right), N_{g s}$ with $N_{s c}$, the number of scientific directions, and $N_{s c}$ with $N_{d m}$, the number of deformable mirrors in the system. $G_{a}$-matrix of the MCAO system also has block structure with $N_{s c} \times N_{d m}$ blocks and each $(s, m)$-block representing the action of $m^{\text {th }} \mathrm{DM}$ on the $s^{\text {th }}$ WFS.

\subsection{Temporal discretization.}

The temporal dynamics of the $\vec{\phi}(t), \vec{s}(t)$ and $\vec{a}(t)$ vectors are discrete owing to the discrete readout from a Coupled Charge Device (CCD) normally used in WFS. CCD integration time is the natural clocking period of the AO controller that specifies the speed requirements for all hardware involved. Thus, the temporal discretization is done by sampling the time-dependent vectors with sampling interval $\triangle t$ equal to integration time of the CCD used in WFS. In particular, the WFS measurement at time $t$ is

$$
\vec{s}_{t}=\int_{t-\triangle t}^{t} \vec{s}(t) d t \approx \vec{s}(t) \triangle t
$$

where subscript " $t$ " stands now for the discrete time. The sampling rate is assumed to be high enough to correctly represent the turbulence continuous temporal behavior according to the Nyquist criterion, that is, it should be greater than twice Greenwood frequency for atmospheric turbulence observed on the astronomical site. In practice the sampling frequency is chosen to be much larger than $2 f_{G}$ in order to reduce the time delay between measurement and control thus maintaining the AO system stable, the issue, which will be carefully addressed in the next chapters. 


\subsection{Performance estimation.}

In this section the discretized model for the basic MCAO system performance metrics will be described. The main quantity all performance metrics can be derived from is the instantaneous residual phase distribution in the main aperture

$$
\epsilon_{t}\left(\vec{x}_{a}\right)=\phi_{t}^{\text {turb }}\left(\vec{x}_{a}\right)-\phi_{t}^{d m}\left(\vec{x}_{a}\right),
$$

where $\phi_{t}^{\text {turb }}$ and $\phi_{t}^{d m}$ are the turbulence phase and the DM correction projected onto the main aperture. Given $\epsilon_{t}\left(\vec{x}_{a}\right)$, the instantaneous optical field distribution in the focal plane of the telescope (instantaneous coherent impulse response $h_{t}\left(\vec{x}_{a}\right)$ ) can be found by the Fraunhofer diffraction integral

$$
h_{t}\left(\vec{x}_{f}\right)=\frac{1}{i \lambda f} \exp \left[\frac{k}{2 f}\left|\overrightarrow{x_{f}}\right|^{2}\right] \int_{S} \exp \left[\epsilon\left(\overrightarrow{x_{a}}\right)\right] \exp \left[i \frac{2 \pi}{\lambda f} \vec{x}_{f} \cdot \vec{x}_{a}\right] d \vec{x}_{a},
$$

which is just a scaled Fourier transform of the main aperture field distribution. In Eq. (3.27), $f$ is the telescope's focal distance, $\vec{x}_{a}, \vec{x}_{f}$ are the aperture and focal plane coordinates, $\lambda$ the observation wavelength. Average point-spread function (PSF) over exposure time

$$
\left\langle\left|h\left(\vec{x}_{f}\right)\right|^{2}\right\rangle_{t}=\sum_{t=1}^{N_{\text {exp }}}\left|h_{t}\left(\vec{x}_{f}\right)\right|^{2}
$$

gives an approximation to the average PSF (2.24) and, thus, Strehl ratio. The Fourier transform of $\left\langle\left|h\left(\vec{x}_{f}\right)\right|^{2}\right\rangle_{t}$ gives an approximation to the system OTF (2.23).

It is obvious from Eq. (3.27) that piston fraction of $\epsilon\left(\overrightarrow{x_{a}}\right)$ results in just constant phase factor in the focal plane and thus does not affect system's blur. In order for the aperture phase residual itself to be a good measure of system's performance the piston term should be subtracted resulting in the piston-removed aperture phase residual

$$
\tilde{\epsilon}\left(\vec{x}_{a}\right)=\epsilon\left(\vec{x}_{a}\right)-\frac{1}{|S|} \int_{S} \epsilon\left(\vec{x}_{a}\right) d \vec{x}_{a},|S|=\int_{S} d \vec{x}_{a},
$$


With the aid of Eqs. (3.10) and (3.25) we can write for the residual phase $\epsilon_{l}$ in the direction of the $l^{\text {th }}$ scientific target

$$
\epsilon_{l}\left(\vec{x}_{a}\right)=\sum_{k=1}^{N_{s c r}} \sum_{i=1}^{N_{k}} \phi_{k, i} f_{l k, i}\left(\vec{x}-\vec{\theta}_{l} h_{k}^{p s}\right)-\sum_{m=1}^{N_{d m}} \sum_{j=1}^{M_{m}} a_{m, j} r_{l m, j}\left(\vec{x}-\vec{\theta}_{l} h_{m}^{d m}\right) .
$$

A convenient MCAO system performance criterion, which can be used as a merit function in optimization process, is the aperture residual phase variance integrated over the aperture and field of view. With $N_{s c}$ discrete scientific directions considered the integrated residual phase variance is

$$
\sigma_{\epsilon}^{2}=\frac{1}{|S|} \sum_{l=1}^{N_{s c}} w_{i} \int_{S} d \overrightarrow{x_{a}}\left\langle\tilde{\epsilon}_{l}^{2}\left(\vec{x}_{a}\right)\right\rangle, \sum_{l=1}^{N_{s c}} w_{l}=1,
$$

where $\left\{w_{l}\right\}_{l=1}^{N_{s c}}$ are the relative weights assigned to each scientific direction, \langle\rangle defines the average over the joint statistics of the atmospheric turbulence and measurement noise. Substitution of Eq. (3.28) and simplification gives the expression for $\sigma_{\epsilon}^{2}$ in the form

$$
\sigma_{\epsilon}^{2}=\left\langle\left[\begin{array}{c}
\vec{\phi} \\
\vec{a}
\end{array}\right]\left[\begin{array}{cc}
\tilde{R}_{\phi \phi} & \tilde{R}_{\phi a} \\
\tilde{R}_{a \phi} & \tilde{R}_{a a}
\end{array}\right]\left[\begin{array}{ll}
\vec{\phi}^{T} & \vec{a}^{T}
\end{array}\right]\right\rangle
$$

where $\tilde{R}$ is the piston-removed Gramm matrix of the function system $\left\{f\left(\vec{x}_{a}\right), r\left(\vec{x}_{a}\right)\right\}$, whose blocks are:

- Phase grammian

$$
\begin{gathered}
\tilde{R}_{\phi \phi}=R_{\phi \phi}-\Phi \Phi^{T} \\
R_{\phi \phi}=\operatorname{diag}\left(w_{1} R_{\phi \phi}^{1}, \ldots, w_{N_{s c}} R_{\phi \phi}^{N_{s c}}\right), \\
\left(R_{\phi \phi}^{l}\right)_{k k^{\prime}, i^{\prime}}=\frac{1}{|S|} \int_{S} d \vec{x} f_{l k, i}\left(\vec{x}-\vec{\theta}_{l} h_{k}^{p s}\right) f_{l k^{\prime}, i^{\prime}}\left(\vec{x}-\vec{\theta}_{l} h_{k^{\prime}}^{p s}\right), \\
\Phi_{k, i}=\operatorname{diag}\left(w_{1} \Phi^{1}, \ldots, w_{N_{s c}} \Phi^{N_{s c}}\right), \\
\Phi_{k, i}^{l}=\frac{1}{|S|} \int_{S} f_{l k, i}\left(\vec{x}-\overrightarrow{\theta_{l}} h_{k}^{p s}\right) d \vec{x} .
\end{gathered}
$$


- DM grammian

$$
\begin{gathered}
\tilde{R}_{a a}=R_{a a}-A A^{T}, \\
R_{a a}=\operatorname{diag}\left(w_{1} R_{a a}^{1}, \ldots, w_{N_{s c}} R_{a a}^{N_{s c}}\right), \\
\left(R_{a a}^{l}\right)_{m m^{\prime}, j j^{\prime}}=\frac{1}{|S|} \int_{S} d \vec{x} r_{l m, j}\left(\vec{x}-\vec{\theta}_{l} h_{m}^{d m}\right) r_{l m^{\prime}, j^{\prime}}\left(\vec{x}-\vec{\theta}_{l} h_{m^{\prime}}^{d m}\right), \\
A_{m, j}=\operatorname{diag}\left(w_{1} A^{1}, \ldots, w_{N_{s c}} A^{N_{s c}}\right), \\
A_{m, j}^{l}=\frac{1}{|S|} \int_{S} r_{l m, j}\left(\vec{x}-\vec{\theta}_{l} h_{m}^{d m}\right) d \vec{x} .
\end{gathered}
$$

- Phase-DM cross grammian

$$
\begin{gathered}
\tilde{R}_{\phi a}=R_{\phi a}-\Phi A^{T} \\
R_{\phi a}=R_{a \phi}^{T}=\operatorname{diag}\left(w_{1} R_{\phi a}^{1}, \ldots, w_{N_{s c}} R_{\phi a}^{N_{s c}}\right), \\
\left(R_{\phi a}^{l}\right)_{k m, i j}=\frac{1}{|S|} \int_{S} d \vec{x} f_{l k, i}\left(\vec{x}-\vec{\theta}_{l} h_{k}^{p s}\right) r_{m, j}\left(\vec{x}-\vec{\theta}_{l} h_{m}^{d m}\right) .
\end{gathered}
$$

Gramm matrix $R$ is one more fundamental quantity that characterizes a MCAO system. This matrix is symmetric positive semi-definite. Its singular values quantify the amount of coupling between the phase on phase screens or on deformable mirrors and the accumulated phase on the main aperture. Some of the singular values being small or zero means that there exist some phase or DM shape configurations that either do not, or only weakly affect the residual phase in the aperture. These shape configurations, or modes, are given by the corresponding singular vectors of the Gramm matrix. The turbulence phase modes (singular vectors of $R_{\phi \phi}$ ) that do not affect the aperture phase are called unseen modes of the system. Any amount of wave front error that is a linear combination of the unseen modes is exactly cancelled in the aperture plane and does not deteriorate system's performance. Nevertheless, since the MCAO system compensates turbulence in the volume, it will attempt to compensate the unseen modes, which may result in excessive amount of control or even instability. 
The analogous DM modes (singular vectors of $R_{a a}$ ) are called uncontrollable, and have the same negative impact on the MCAO system. Unfortunately, the significant number of unseen and uncontrollable modes are usually present in the MCAO systems, which presents a serious difficulty for the designer. On the other hand, the third kind of modes, the ones belonging to the null space of the full $R$-matrix will show that there exist combinations of turbulence and phase shapes that exactly cancel out the aperture residual, which means that at least part of the turbulence can be compensated by DMs with zero fitting error.

Computation of the Gramm matrix elements given by Eqs. (3.31) - (3.33) requires a large number of double integrals to be evaluated. An approximation of the Gramm matrix that is easier to compute can be done in the following way. Let the residual phase in the aperture be approximated with some set of basis functions $\left\{a_{i}\right\}_{i=1}^{\infty}$

$$
\epsilon_{l}\left(\vec{x}_{a}\right) \approx \sum_{i=1}^{N_{a}} \epsilon_{l, i} a_{i}\left(\vec{x}_{a}\right),
$$

e.g. bilinear splines. The piston-removed aperture and FoV averaged residual phase variance (3.29) is thus approximated as

$$
\sigma_{\epsilon}^{2} \approx\left\langle\vec{\epsilon}^{T} \tilde{W} \vec{\epsilon}\right\rangle
$$

where $\vec{\epsilon}$ is the concatenated vector of approximation coefficients in Eq. (3.34),

$$
\begin{gathered}
\tilde{W}=W-V V^{T}, \\
W=\operatorname{diag}\left(w_{1} W^{1}, \ldots, w_{N_{s c}} W^{N_{s c}}\right), \\
\left(W^{l}\right)_{i j}=\frac{1}{|S|} \int_{S} d \vec{x}_{a} a_{i}\left(\vec{x}_{a}\right) a_{j}\left(\vec{x}_{a}\right), \forall l, l=1, \ldots N_{s c} \\
V=\operatorname{diag}\left(w_{1} V^{1}, \ldots, w_{N_{s c}} V^{N_{s c}}\right), \\
\left(V^{l}\right)_{i}=\frac{1}{|S|} \int_{S} d \vec{x}_{a} a_{i}\left(\vec{x}_{a}\right), \forall l, l=1, \ldots N_{s c} .
\end{gathered}
$$


Note that $W^{l}$ - and $V^{l}$-matrices are the same for all blocks, and, owing to the shiftinvariance property of the functions $a_{i}\left(\vec{x}_{a}\right)$ normally used as a basis set, $W^{l}$ - and $V^{l}$-matrices have lots of equal elements, which greatly simplifies their calculation.

Further, let the approximations of the phase screen basis functions $f_{l k, i}\left(\vec{x}_{a}\right)$ and DM influence functions $r_{l m, j}\left(\vec{x}_{a}\right)$ projected onto the aperture be

$$
\begin{gathered}
f_{l k, i}\left(\vec{x}_{a}\right) \approx \sum_{p=1}^{N_{a}} f_{l k, i p} a_{p}\left(\vec{x}_{a}\right), \\
r_{l m, j}\left(\vec{x}_{a}\right) \approx \sum_{p=1}^{N_{a}} r_{l m, j p} a_{p}\left(\vec{x}_{a}\right) .
\end{gathered}
$$

Then, according to Eq. (3.28), the coefficients in the aperture residual phase approximation (3.34) can be expressed in matrix form

$$
\vec{\epsilon}=H_{\phi} \vec{\phi}-H_{a} \vec{a}
$$

where the elements of phase-to-phase interaction matrix $H_{\phi}$ and DM-to-phase interaction matrix $H_{a}$ are

$$
\begin{gathered}
\left(H_{\phi}\right)_{l k, i p}=f_{l k, i p}, \\
\left(H_{a}\right)_{l m, j p}=r_{l m, j p} .
\end{gathered}
$$

These elements can also be easily computed especially in the case of the spline functions taken as the basis sets for all the phase expansions. The computational ease, however, comes for the price of quite a large $H$-matrix, for it is necessary to take enough terms to obtain a reasonably good approximation in Eqs. (3.37) and (3.38). Thus, the convenient discretized model for the integrated phase variance is

$$
\sigma_{\epsilon}^{2}=\left\langle\left(H_{\phi} \vec{\phi}-H_{a} \vec{a}\right)^{T} \tilde{W}\left(H_{\phi} \vec{\phi}-H_{a} \vec{a}\right)\right\rangle
$$

which is the main result of this section. 


\subsection{Phase reconstructors.}

From the statistical estimation theory standpoint, since the atmospheric turbulence is believed to be a zero mean, stationary, Gaussian random process, an optimal phase estimator is supposed to be linear time invariant [64]. AO control is a process of finding the DM command vector $\vec{a}_{t}$ at time $t$ by application of some linear time invariant operator $R$ (matrix in discretized case) to the set of current and past measurements $\vec{S}_{t}=\left[\vec{s}_{t} \vec{s}_{t-1 \ldots}\right]^{T}$ of the atmospheric turbulence $\phi_{t}$ :

$$
\vec{a}_{t}=\hat{R} \vec{S}_{t}
$$

such that to minimize the discretized $\mathscr{H}_{2}$-norm of the aperture phase residual $\left(\mathscr{H}_{2^{-}}\right.$ control $[13,66])$ :

$$
\hat{R}=\arg \min _{R}\left\langle\left(H_{\phi} \vec{\phi}_{t}-H_{a} R \vec{S}_{t}\right)^{T} \tilde{W}\left(H_{\phi} \vec{\phi}_{t}-H_{a} R \vec{S}_{t}\right)\right\rangle
$$

where $H_{\phi}, H_{a}, \tilde{W}$ are the matrices defined in the previous section. The alternative way to find the optimal reconstructors is to minimize the $\mathscr{H}_{\infty}$ norm of the phase residual $\left(\mathscr{H}_{\infty}\right.$-control $\left.[32,66]\right)$

$$
\left\|\epsilon\left(\vec{x}_{a}\right)\right\|_{\infty}=\max _{\vec{x}_{a} \in S}\left|\epsilon\left(\vec{x}_{a}\right)\right|
$$

but this is beyond the scope of this dissertation.

Three the most important approaches to find the optimal reconstructor $\hat{R}$ considered in adaptive optics are

- Least Squares (LS)

- Non-dynamic Minimum Variance (MV)

- Dynamic Minimum Variance or Kalman Filter (KF) 


\subsubsection{Least Squares (LS) reconstructor.}

The LS reconstructor is the simplest and the most widely used in conventional adaptive optics reconstructor. It is computed in the following way.

- Only current measurement, $\vec{s}_{t}$, is taken to compute the current DM command vector $\vec{a}_{t}$, which is referred to as non-dynamic wave front reconstruction.

- No turbulence statistics are taken into account as an a priori knowledge.

- Instead of variational problem (3.44) the simpler problem of minimization the residual between the current measurement and the actuator command action read out from the WFS

$$
\hat{a}_{t}^{L S}=\arg \min _{\vec{a}}\left\|\vec{s}_{t}-G_{a} \vec{a}\right\|^{2}
$$

is solved, which yields

$$
\hat{R}^{L S}=G_{a}^{\dagger},
$$

where $G_{a}^{\dagger}$ is the pseudo-inverse of the DM-to-WFS interaction matrix usually defined through the Truncated Singular Value Decomposition of $G_{a}$ (TSVD):

$$
G_{a}^{\dagger} \vec{s}=\sum_{n=1}^{N_{t r}} \sigma_{n}^{-1} \vec{v}_{n} \vec{u}_{n}^{T} \vec{s},
$$

where $\sigma_{n}, \vec{u}_{n}, \vec{v}_{n}$ are the singular values, left and right singular vectors of $G_{a}$, respectively, $N_{t r}$ is the number of nonzero singular values kept, which is always less than the size of $G_{a}$ because of the non-observable modes present. Another way to compute the pseudo-inverse, which may lead to low computational complexity algorithms, is Tikhonov regularization, when the minimization problem (3.45) is modified as

$$
\hat{a}_{\alpha, t}^{L S}=\arg \min _{a}\left\|\vec{s}_{t}-G_{a} \vec{a}+\alpha \vec{s}_{t}^{T} L \vec{s}_{t}\right\|^{2},
$$


where $L$ and $\alpha$ are the regularization operator and regularization parameter that damp the singularity of $G_{a}$ and yield the alternative form of LS reconstructor matrix

$$
\hat{R}_{\alpha}^{L S}=G_{a, \alpha}^{\dagger}=\left(G_{a} G_{a}^{T}+\alpha L\right)^{-1} G_{a}^{T}
$$

The advantages of the Least Squares approach are its simplicity and relatively low computational complexity. On the other hand, since the usually available a priori information about turbulence and measurement statistics is not taken into account, LS reconstructor is not optimal with respect of giving the smallest residual error. However, simple modifications such as the clever choice of the regularization operator $L$ [45], can be made to significantly improve the quality of LS reconstructor.

\subsubsection{Minimum Variance reconstructor.}

To obtain the non-dynamic MV reconstructor we again set $\vec{S}_{t}=\vec{s}_{t}$, that is just the current measurement. Solving the minimization problem (3.44) in this case yields [47]

$$
\hat{R}^{M V}=\hat{F} \hat{E}
$$

where the estimation $\hat{E}$ and fitting $\hat{F}$ matrices are defined as

$$
\begin{gathered}
\hat{E}=C_{\phi \phi} G_{\phi}^{T}\left(G_{\phi} C_{\phi \phi} G_{\phi}^{T}+C_{n n}\right)^{-1} \\
=\left(G_{\phi} C_{n n}^{-1} G_{\phi}^{T}+C_{\phi \phi}^{-1}\right)^{-1} G_{\phi}^{T} C_{n n}^{-1}, \\
\hat{F}=\left(H_{a} \tilde{W} H_{a}^{T}+\alpha L\right)^{-1} H_{a}^{T} \tilde{W} H_{\phi},
\end{gathered}
$$

$L, \alpha$ are the regularization operator and regularization coefficient to account for the unseen and uncontrollable modes.

It is not difficult to see that the estimation and fitting matrices can be defined as solutions of two independent deterministic minimization problems: 
1. Optimal phase estimation

$$
\hat{\vec{\phi}}_{t}=\hat{E} \vec{s}_{t}=\arg \min _{\vec{\phi}}\left(\left\|\vec{s}_{t}-G_{\phi} \vec{\phi}\right\|_{C_{n n}^{-1}}^{2}+\|\vec{\phi}\|_{C_{\phi \phi}^{-1}}^{2}\right)
$$

2. Optimal fitting of the DM commands to the phase estimate

$$
\hat{\vec{a}}_{t}=\hat{F} \hat{\vec{\phi}}_{t}=\arg \min _{\vec{a}}\left(\left\|H_{\phi} \hat{\vec{\phi}}_{t}-H_{a} \vec{a}\right\|_{\tilde{W}}^{2}+\alpha\|\vec{a}\|_{L}^{2}\right),
$$

where a weighted norm of the vector is defined as

$$
\|\vec{v}\|_{W}^{2}=\vec{v}^{T} W \vec{v}
$$

Note that the first minimization problem deals with all the statistical priors involved in the problem, whereas the second one has to do only with AO system geometry. The possibility to split the minimization problem into two independent parts is known as the Separation Principle of the $\mathscr{H}_{2}$-control [66].

Because of the additional a priori information about turbulence and noise statistics involved, which comes in the form of covariance matrices, minimum variance approach provides lower reconstruction mean square error. As it is seen from the Eq. (3.51) the $C_{\phi \phi}$-matrix serves as a Tikhonov regularization operator and removes the unobservable modes from the null space of matrix $G_{\phi}$. Minimum Variance reconstructor is currently considered the most promising control algorithm for the new generation of the AO systems. More careful study of the MV reconstructor and its modifications is presented in Chapters 4 and 6.

\subsubsection{Kalman Filter.}

The Kalman Filter, whose detailed description will be presented in Chapter 5, is known as a dynamic statistical estimation method, which, in order to get the current turbulence phase estimate, uses both current and all the past measurements. In addition 
to the turbulence and measurement noise second order statistics also used in MV, KF also employs a linear autoregressive model, the crucial part of Kalman filter approach, used to describe the turbulence dynamics:

$$
\vec{X}_{t+1}=A \vec{X}_{t}+\vec{w}_{t+1}
$$

where the state $\vec{X}_{t}$ is a concatenation of the current and some past phase vectors $\vec{\phi}_{t}$, $A$ is the state transition matrix and $\vec{w}_{t+1}$ is the vector of driving noise assumed to be zero mean temporally white with known spatial covariance matrix $C_{w w}$ and uncorrelated with the measurement noise $\vec{n}_{t}$. This dynamics model enables an approximate prediction of the turbulence time evolution, which can be effectively used for reduction of the temporal error (see Sec. 2.5.2).

To obtain Kalman Filter reconstructor we set $\vec{S}_{t}$ to be equal to a concatenation of the current and all past measurements

$$
\vec{S}_{t}=\left[\vec{s}_{0} \ldots \vec{s}_{t}\right]^{T} .
$$

The optimal estimator $\hat{R}_{t}^{K F}$ then depends on time because of growing size of the input data set employed at each time step. The separation principle also holds for the Kalman Filter $[56,66]$ and results in the minimization problem in Eq. (3.44) being split into

1. Fitting problem in the form of Eq. (3.54).

2. Kalman estimation problem, which is normally written in the form of the orthogonality principle

$$
\left\langle\left(\vec{\phi}_{t}-\hat{E}_{t}^{K F} \vec{S}_{t}\right) \vec{S}_{t}^{T}\right\rangle=0,
$$

which, together with Eq. (3.56) is used to derive the optimal Kalman estimator $\hat{E}_{t}^{K F}$. 
Since the Kalman Filter is derived from formally the same variational principle the non-dynamic MV reconstructor is based on, KF can be considered the best possible minimum variance estimator for it takes into account all measurements available.

\subsection{Monte-Carlo simulations.}

Because of the high complexity of MCAO systems analysis and the fact that the inputs of the AO system, the turbulence-induced phase errors and measurement noise, are stochastic processes the most convenient way to assess the system's performance is through Monte-Carlo simulations. Another motivation to use Monte-Carlo simulations is the fact that, despite the linearity assumptions made for all of the MCAO system elements, the atmospheric turbulence itself is not a linear process, i.e. it cannot be modelled as the output of some linear dynamic system driven by the white noise. If it were a case, the time-consuming Monte-Carlo simulations would be avoided and all the MCAO system performance metrics, such as aperture phase residual $\vec{\epsilon}\left(\vec{x}_{a}\right)$ and DM control vector $\vec{a}_{t}$ dynamics could be found theoretically using the tools of the linear system analysis [66].

The main steps of the Monte-Carlo simulation of an AO system can be summarized in the form of the following algorithm.

\section{Monte-Carlo simulation of the MCAO system}

- BEGIN

- Load the atmosphere parameters: $C_{n}^{2}(z), \vec{v}(z), L_{0}, l_{0}$.

- Use the algorithm described in Sec. 3.1 to create the layered model of the atmosphere, compute the integrated turbulence parameters $r_{0}, \theta_{0}, f_{G}$ using the corresponding equations from Secs. 2.2, 2.3. 
- Specify the AO system parameters: primary and secondary mirrors' diameters, number and geometry of DMs and WFSs, WFS noise levels and integration time, scientific and guide stars' altitudes, directions and types.

- Compute the fundamental system's matrices $G_{\phi}, G_{a}, H_{\phi}, H_{a}, \tilde{W}$ using the corresponding equations of this chapter.

- Compute the reconstruction matrix $\hat{R}$.

- Begin Monte-Carlo simulation loop

* Generate random phase pattern $\vec{\phi}_{t}$ on the phase screens according to the statistical specifications $\left(r_{0}, \mathrm{PSD}\right)$ for each screen.

* Generate WFS readout $\vec{s}_{t}$ using Eq. (3.21).

* Apply a delay between the turbulence phase and WFS measurement to model the time taken by the real measurement and reconstruction precesses.

* Apply the reconstructor to the delayed WFS measurement vector generated on the previous step to obtain the current DM commands vector $\hat{\vec{a}}_{t}$.

* Find the instant aperture phase residual vector $\vec{\epsilon}_{t}$ by Eq. (3.39).

* Compute the instant PSF, OTF, Strehl ratio and the residual phase variance by equations given in Sec. 3.5.

* Accumulate the instant values of performance metrics to obtain the long exposure PSF, OTF, Strehl ratio and the residual phase variance.

- End Monte-Carlo simulation loop

- $E N D$

The described Monte-Carlo simulations must be repeated many times for different statistical realizations of the atmospheric turbulence and the corresponding performance 
metrics must be averaged to obtain the approximations for their statistical mean values.

The simulation process has two random inputs: measurement noise and turbulence phase. Since the WFS noise is supposed to be Gaussian temporally and spatially uncorrelated, its generation does not present any difficulty. On the other hand, high fidelity turbulence phase generation is the nontrivial problem. To be more specific, it is necessary to generate the random coefficients vectors $\vec{\phi}_{t}$ in the turbulence phase approximation given by Eq. (3.5), which are Gaussian zero-mean random variables with the covariance matrix specified by Kolmogorov or von Karman statistics. Without going into details we mention that the most widely used methods of random turbulence phase generation are the following. Generation of random coefficients of the phase approximation by Zernike polynomials can be done by the method described in [33]. In the Fourier domain $[38,39]$ and fractal-based [40] turbulence phase generation methods the random phase values on a set of grid points are generated. According to Eq. (3.9), these values can be taken as the bilinear approximation coefficients of the continuous turbulence phase distributions.

The Fourier domain phase screens generation with tip-tilt correction [41] has been used in the Monte-Carlo simulations performed by the author because of its simplicity and high accuracy. The wind shift of the phase screens according to the Frozen Flow Hypothesis can be modelled by:

- Direct shifting of the discrete phase grid. This is the most accurate method, though it has serious restrictions:

- In order to avoid interpolation and thus loose accuracy, only the shifts, which are multiples of the grid spacing, are allowed.

- Direction of the wind should be along the grid lines, which is not a problem for SCAO, where the laboratory coordinate system orientation can always 
be chosen to coincide with the wind direction, but is a problem for MCAO, where several phase screens are moving in different directions.

- Fourier domain shift by means of the shift theorem for the Fourier transform

$$
\phi(\vec{x}-\vec{v} \triangle t)=\mathscr{F}^{-1}\{\exp [-i k \Delta t \vec{v} \cdot \vec{f}] \mathscr{F}\{\phi(\vec{x})\}\}
$$

where $\vec{x}, \vec{f}$ are the spatial and frequency domain coordinates, $\vec{v}$ the wind speed vector, $\triangle t$ the sampling time interval, $k=2 \pi / \lambda$ the wave number. This model allows shifts of any magnitude and direction but, when discretized, Eq. (3.59) is only an approximation and may introduce significant error.

One more important comment about the Monte-Carlo simulations is that the phase estimate $\hat{\vec{\phi}}$ made with the aid of the wave front reconstructor always has lower resolution than the input phase screen generated, that is, the number of basis functions in Eq. (3.5) taken to approximate the phase estimate is smaller than number of functions to approximate the input phase. The phase estimate resolution and, therefore, its spectral content, should be taken equal or higher than the DM actuator grid resolution to generate an adequate DM commands on the fitting step. On the other hand, the phase screens that are the input of the Monte-Carlo simulation are generated with much higher resolution to represent higher spatial frequencies present in real turbulence that are beyond the pass-band of the AO system. This allows to evaluate the error induced by the uncompensated fraction of the turbulence. In order to find the right resolution of the input phase screens we note that inner scale $l_{0}$ of the turbulence gives the size of the smallest detail in phase patterns. With $l_{0}=1 \mathrm{~mm}$ on the turbulence ground layer [33] and two samples taken to represent the smallest detail, the resolution of the phase screen should equal $2 \mathrm{~mm}^{-1}$, which makes $400 \mathrm{~K}$ grid points for a $10 \times 10 \mathrm{~m}$ square phase screen and is far beyond the current computational limitations. On the other hand, because of the fast $(-11 / 3)$-power roll-off of the turbulence phase power 
spectrum, the highest frequency components make very small contribution and quite accurate representation of the input turbulence can be done by discrete phase screens with relatively low resolution. The empirical rule to choose the input phase screens resolution used in the simulations presented below is the following: the reconstruction error of an AO system is estimated by a sequence of Monte-Carlo simulations with input phase screens of increasing resolution and the process is stopped at the resolution above which the reconstruction error change is no more than $10 \%$. It has been found by trial and error that having the input phase screens resolution eight times bigger than that of the finest DM actuator grid works well enough for all cases. It also needs to be mentioned that, in order to correctly represent the aperture phase residual (3.42), the resolution of the aperture phase grid should be at least twice the highest resolution of the phase screens. Having two sets of phase screens with different resolution for the reconstruction and for the simulation input implies the necessity to have two sets of the system matrices: $G_{\phi}, H_{\phi}$-matrices computed on the high resolution grids and used for performance estimation and the same set of matrices computed on the lower resolution grids and used to build the reconstructor.

More detailed description and modifications of the mathematical approach to model MCAO systems described in this chapter will be used below in connection with analysis of several important special cases of AO control. 


\section{CHAPTER 4}

\section{Robustness study of the sparse non-dynamic Minimum Variance controller}

The Minimum Variance (MV) control briefly described in Sec. 3.6.2 is one of the most appealing approaches for the next generation of large scale AO. The two most important features that make MV more advantageous in comparison to other approaches are:

- The use of a priori information about atmospheric turbulence statistics in the form of covariance matrices improves the quality of turbulence compensation.

- It is possible to implement MV algorithm in sparse form with low computational complexity.

Significant progress has been made in the development of MV algorithm both in modal [51] and zonal [36] representations. In the theoretical aspect, MV approach has reached the maturity level making it applicable for use in real AO systems, and there comes a time to investigate some aspects of its practical implementation. One of these aspects is the degree of robustness of the MV algorithm, that is, its sensitivity to the errors and model uncertainties present in any real AO system. This chapter is the account of the work made to quantify the degree of MV controller performance deterioration as a function of various system errors. Section 4.1 describes low complexity implementation of the MV algorithm, the core of the MV approach for large scale AO. 
Section 4.2 gives a brief theoretical description of the Pseudo Open-Loop algorithm, the modification of MV control for closed-loop AO system operation, and methods for its stability and performance analysis. Section 4.4 presents the results of this analysis applied to the case of the Gemini-South $8 \mathrm{~m}$ telescope MCAO system. Conclusions are given in Section 4.5.

\subsection{Low complexity implementation of the MV reconstruction algorithm.}

From the computational standpoint, process of linear control of an AO system is a sequence of matrix-vector multiplications. If the reconstruction matrix is fully populated, as it is the case for the TSVD LS reconstructor given by Eq. (3.47), a matrix-vector multiplication can be done at the $\mathscr{O}\left(N^{2}\right)$ cost, where $N$ is the size of the matrix. On the other hand, doing real time wave front reconstruction at the $\mathscr{O}\left(N^{2}\right)$ cost for a giant telescope AO system with $N \sim 10^{5}$ degrees of freedom is computationally impossible at this time. Finding the $\mathrm{AO}$ control algorithms with complexity lower than $\mathscr{O}\left(N^{2}\right)$ is one of the biggest challenges for the next generation of AO systems.

Complexity reduction of the control algorithms is possible, if the quite favorable internal structure of the MCAO system model is taken into account. In the framework of zonal approach all the basis functions to approximate phase distributions on phase screens (Eq. (3.5)), aperture basis functions (Eq. (3.34)) and DM influence functions (Eq. (3.25)) are considered to be localized ones. With such functions all the system matrices become very sparse. For instance, each row of the phase-to-WFS interaction matrices $G_{a, \phi}$ has the number of nonzero elements equal to the number of splines falling fully or partially into a single WFS aperture area, each row of the phase-tophase interaction matrices $H_{a, \phi}$ contains no more than four nonzero elements and each 
row of the aperture weighting matrix $W$ contains no more than nine nonzero elements. The sparsity patterns of the $G$-, $H$ - and $W$-matrices for the representative case of the Gemini South 8-meter telescope MCAO system are shown of Fig. 4.1. Taking into account that the size of the system matrices is a small multiple of the MCAO system's number of degrees of freedom, we can conclude that the number of nonzero elements in the matrices, which actually determines the computational complexity of the matrix manipulations, is $\mathscr{O}(N)$.

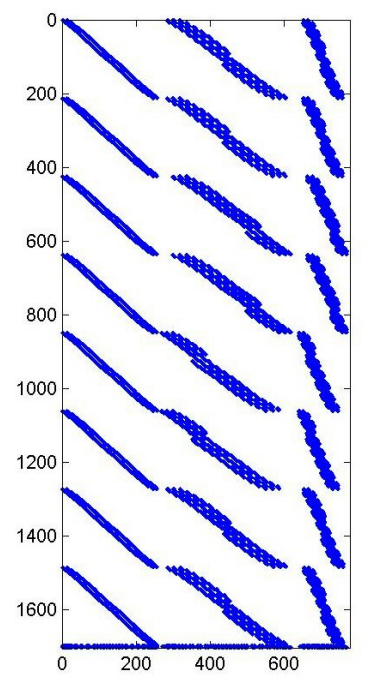

(a)

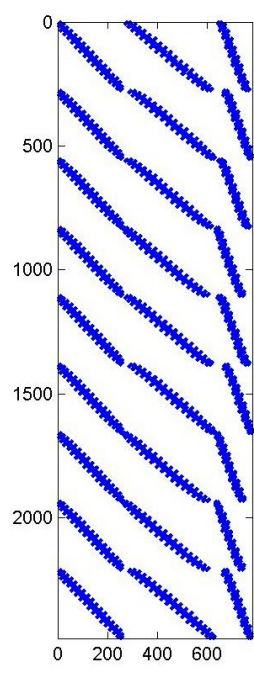

(b)

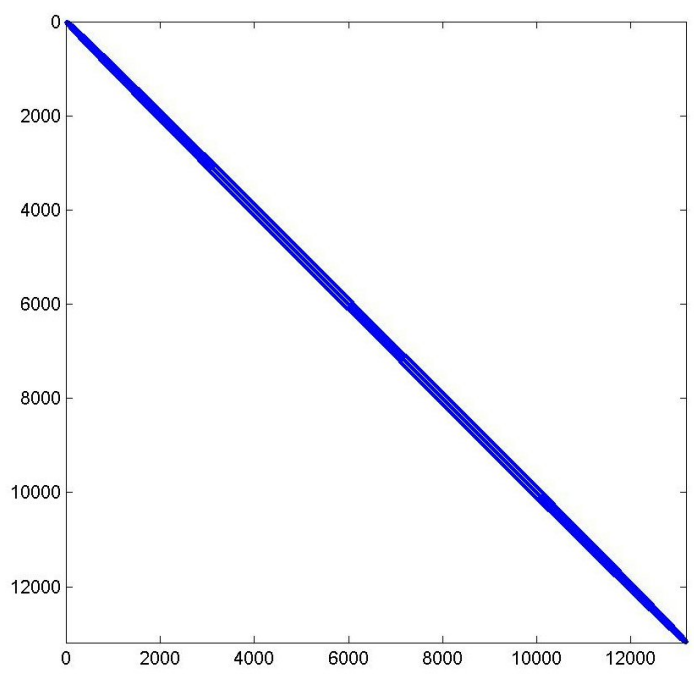

(c)

Figure 4.1: Sparsity patterns of the $G_{a^{-}}$(a), $H_{a^{-}}$(b) and $W$-matrices (c) of the model for the Gemini South 8-meter telescope MCAO system. The filling factors are $2.49 \%$, $0.07 \%$ and $1.5 \%$, respectively.

This makes it possible, with the aid of sparse matrix numerical methods, to create MV-based AO control algorithms with nearly $\mathscr{O}(N)$ complexity. The idea of sparse implementation of the MV reconstructor belongs to B. L. Ellerbroek [36], and is briefly described below.

We repeat here for clarity Eqs. (3.51) and (3.52) that make the minimum variance 
estimator $\hat{R}^{M V}=\hat{F} \hat{E}^{M V}$ :

$$
\begin{aligned}
\hat{E}^{M V} & =\left(G_{\phi} C_{n n}^{-1} G_{\phi}^{T}+C_{\phi \phi}^{-1}\right)^{-1} G_{\phi}^{T} C_{n n}^{-1}, \\
\hat{F} & =\left(H_{a} \tilde{W} H_{a}^{T}+\alpha I\right)^{-1} H_{a}^{T} \tilde{W} H_{\phi},
\end{aligned}
$$

where

- $G_{\phi}$ is a phase-to-WFS interaction matrix described in Sec. 3.2;

- $H_{\phi}, H_{a}, \tilde{W}$ are phase-to-phase, phase-to-DM interaction and aperture weighting matrices described in Sec. 3.5;

- $C_{\phi \phi}, C_{n n}$ are the phase and WFS noise covariance matrices described in Secs. 3.1 and 3.2 , respectively;

- simple energy constraint $L=I$ is taken for the regularization operator in the fitting matrix equation.

Inspection of the estimation and fitting operators shows that they consist of the mixture of sparse and non-sparse matrices. The non-sparsity comes from three different sources:

1. Non-sparse phase covariance matrix $C_{\phi \phi}$ and its inverse as it is seen from Eqs. (3.17) or (3.18).

2. Low-rank terms in the form $\vec{u} \vec{v}^{T}$ due to piston or point removal operation that affect the phase and LGS noise covariance matrices as well as the aperture weighting matrix.

3. Presence of matrix inverses that will turn a sparse matrix into a non-sparse one. 


\subsubsection{Sparse approximation of matrix $C_{\phi \phi}^{-1}$.}

To address the first difficulty mentioned the sparse approximation for matrix $C_{\phi \phi}^{-1}$ has been proposed [36]. The reasoning for it is the following. In the limiting case of the very dense grid on the infinite phase screen the bilinear functional defined by the matrix $C_{\phi \phi}^{-1}$ can be approximated by its continuous counterpart:

$$
\begin{gathered}
\vec{u}^{T} C_{\phi \phi}^{-1} \vec{v}=\vec{u}^{T}\left\langle\vec{\phi}^{T}\right\rangle^{-1} \vec{v} \\
=\iint d \vec{x} d \vec{x}^{\prime} u(\vec{x}) v\left(\vec{x}^{\prime}\right)\left\langle\phi(\vec{x}) \phi^{*}\left(\vec{x}^{\prime}\right)\right\rangle^{-1} \\
=\iint d \vec{k} d \vec{k}^{\prime} \tilde{u}(\vec{k}) \tilde{v}^{*}\left(\vec{k}^{\prime}\right)\left\langle\tilde{\phi}(\vec{k}) \tilde{\phi}^{*}\left(\vec{k}^{\prime}\right)\right\rangle^{-1} \\
=\iint d \vec{k} d \vec{k}^{\prime} \tilde{u}(\vec{k}) \tilde{v}^{*}\left(\vec{k}^{\prime}\right) \Phi^{-1}(\vec{k}) \delta\left(\vec{k}-\vec{k}^{\prime}\right) \\
=\int d \vec{k} \tilde{u}(\vec{k}) \tilde{v}^{*}(\vec{k}) \Phi^{-1}(\vec{k}),
\end{gathered}
$$

where $\vec{x}, \vec{k}$ are spatial and frequency coordinates, $\Phi(\vec{k})$ is the turbulence PSD, the Fourier transform of function $f$ is denoted by $\tilde{f}$. Plancherel theorem [42] is used to switch from spatial domain integration to frequency domain integration and the fact that in the infinite domain the Fourier components of a stationary random process form Karhunen-Loeve basis [65] is used to turn double integration into single one. In order to further simplify Eq. (4.1) we expand the von Karman PSD into Taylor series:

$$
\Phi^{-1}(\vec{k}) \propto\left(|\vec{k}|^{2}+k_{0}^{2}\right)^{11 / 6}=\frac{11}{3} k_{0}^{5 / 3}|\vec{k}|^{2}+\frac{55}{3} k_{0}^{-1 / 3}|\vec{k}|^{4}+\ldots
$$

where $k_{0}=2 \pi / L_{0}, L_{0}$ is the outer scale, whose measured value for good astronomical sites is around $50 \mathrm{~m}$ [63]. This makes $k_{0}$ significantly less than 1 and shows that the $|\vec{k}|^{4}$-term will be predominant in the inverse PSD expansion. Keeping just this term gives

$$
\vec{u}^{T} C_{\phi \phi}^{-1} \vec{v} \propto \int d \vec{k} \tilde{u}(\vec{k}) \tilde{v}^{*}(\vec{k})|\vec{k}|^{4}
$$




$$
=\int d \vec{x} \nabla^{2} u(\vec{x}) \nabla^{2} v^{*}(\vec{x})
$$

i.e. the quantity $\vec{u}^{T} C_{\phi \phi}^{-1} \vec{v}$ is approximately proportional to the inner product of the Laplacians of $\vec{u}$ and $\vec{v}$, thus the inverse phase covariance matrix can be approximated as

$$
C_{\phi \phi}^{-1} \approx \eta L^{T} L
$$

where $L$ is a discrete Laplacian and $\eta$ is the proportionality constant found from normalization condition

$$
\eta\left\|L^{T} L\right\|=\left\|C_{\phi \phi}^{-1}\right\|
$$

The approximation in the form of Eq. (4.4) may seem to be too crude. However, it works very well because since the $C_{\phi \phi}^{-1}$ serves in the equation (3.51) as a regularization term, its influence on the phase estimate is quite weak and thus insensible even to large errors. Fig. 4.2 illustrates the process of the discrete Laplacian or curvature matrix computation. Stars in this figure represent the points of the discrete phase grid. The values near the stars are the nonzero coefficients needed to compute the value of Laplacian at the interior point A or the boundary points B, C. The "fold-over" boundary condition is applied, that is, the coefficient that should be assigned to the point outside the boundary is added to the coefficient assigned to the point opposite to it with respect to the central point, where the curvature is computed, so that the sum of coefficients is made zero. The most important is that 2-D discrete Laplacian defined on a square phase grid is a sparse matrix. This eliminates the non-sparse matrices from the MV reconstructor, making it a combination of sparse matrices, low-rank matrices and their inverses. 


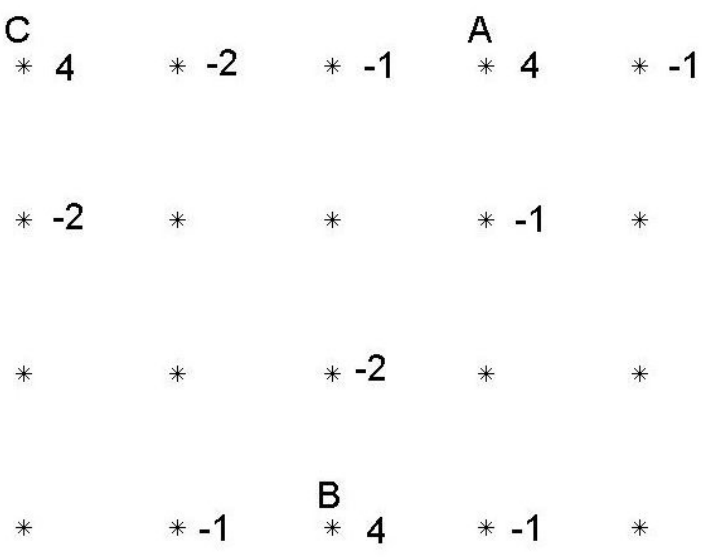

Figure 4.2: Discrete Laplacian or curvature operator [70]. This figure illustrates the coefficients of discrete Laplacian computation. Stars represent the grid points of the discrete phase grid. The values near the stars are the nonzero coefficients needed to compute the value of Laplacian at the interior point A or the boundary points B, C. 


\subsubsection{Fast matrix inverses.}

Explicit computation of the inverse of any matrix will most likely result in fully populated inverse matrix and its multiplication by a vector requires $\mathscr{O}\left(N^{2}\right)$ operations. On the other hand, in the control process we do not need to explicitly specify $\hat{R}^{M V}$, we only need its action $\hat{R}^{M V} \vec{s}$ on the WFS measurement vector, which is a chain of matrix-vector multiplications

$$
\begin{gathered}
\vec{\Psi}_{1}=G_{\phi}^{T} C_{n n}^{-1} \vec{s}, \\
\vec{\Psi}_{2}=\left(G_{\phi}^{T} C_{n n}^{-1} G_{\phi}+\eta L^{T} L\right)^{-1} \vec{\Psi}_{1}, \\
\vec{\Psi}_{3}=\left(H_{a}^{T} \tilde{W} H_{\phi}\right) \vec{\Psi}_{2}, \\
\vec{\Psi}_{4}=\left(H_{a}^{T} \tilde{W} H_{a}+\alpha I\right)^{-1} \vec{\Psi}_{3},
\end{gathered}
$$

It is obvious that multiplication operation (4.8) can be done at the $\mathscr{O}(N)$ cost because of the "sparse + low-rank" form of matrix $H_{a}^{T} \tilde{W} H_{\phi}$. We can also show that the inverse measurement noise covariance matrix $C_{n n}^{-1}$ has "sparse + low-rank" representation owing to its simple internal structure. Indeed, in the case of laser guide star, pistonor point-removed noise covariance matrix $\tilde{C}_{n n}$ is given by Eq. (3.24). This matrix is block-diagonal and each its block has the form $\tilde{C}_{i}=P C_{i} P^{T}$, where $P$ is either piston or point removal projector, $C_{i}$ is the diagonal matrix. Note that $\tilde{C}_{n n}$ is rank-deficient and cannot be directly inverted, so the pseudo-inverse $\tilde{C}_{n n}^{\dagger}$ must be found instead. Piston and point removal cases are treated separately:

- Pseudo-inverse of the $\tilde{C}_{n n}$ blocks in the piston-removed case can be found as

$$
\tilde{C}_{i}^{\dagger}=\left(C_{i}+\overrightarrow{1}^{T}\right)^{-1}
$$

where $\overrightarrow{1}$ is the vector of ones. Applying the matrix inversion lemma

$$
\left(M \mp U V^{T}\right)^{-1}=M^{-1} \pm\left(M^{-1} U\right)\left(I \mp V^{T} M^{-1} U\right)^{-1}\left(M^{-1} U\right)^{T}
$$


we get the "sparse + low-rank" form for the pseudo-inverse

$$
\begin{gathered}
\tilde{C}_{i}^{\dagger}=C_{i}^{-1}+\gamma_{p t} \vec{v} \vec{v}^{T} \\
\vec{v}=C_{i}^{-1} \overrightarrow{1}, \gamma_{p t}=\left(1-\overrightarrow{1}^{T} C_{i}^{-1} \overrightarrow{1}\right)^{-1}
\end{gathered}
$$

- In the point-removed case we have

$$
\tilde{C}_{i}=P_{p t} C_{i} P_{p t}^{T}=C_{i}^{0}+\left(C_{i}\right)_{r r} \overrightarrow{1}^{0}\left(\overrightarrow{1}^{0}\right)^{T}
$$

where $r$ is the reference subaperture number, $C_{i}^{0}$ is the $C_{i}$-matrix with $r^{\text {th }}$ row and column replaced with zeros, $\overrightarrow{1}^{0}$ is the $\overrightarrow{1}$-vector with zero on $r^{\text {th }}$ position. Zero rows and columns can be removed resulting in full-rank matrix. From the matrix inversion lemma its inverse is again in the "sparse + low-rank" form

$$
\begin{gathered}
\tilde{C}_{i}^{r}=\left(C_{i}^{r}\right)^{-1}-\gamma_{p t} \vec{v} \vec{v}^{T}, \\
\vec{v}=\left(C_{i}^{r}\right)^{-1} \overrightarrow{1}^{r}, \gamma_{p t}=\left(1+\left(C_{i}\right)_{r r}\left(\overrightarrow{1}^{r}\right)^{T}\left(C_{i}^{r}\right)^{-1} \overrightarrow{1}^{r}\right)^{-1},
\end{gathered}
$$

where superscript " $r$ " denotes the matrix with $r^{\text {th }}$ row and column removed.

Thus, in both cases matrix $\tilde{C}_{n n}^{\dagger}$ can be represented by only a small number of nonzero elements and the multiplication operation (4.6) can be done at the $\mathscr{O}(N)$ cost.

Doing operations (4.7) and (4.9), that is multiplication by an inverse matrix, is equivalent to solving, correspondingly, the equation systems:

$$
\begin{gathered}
\left(G_{\phi}^{T} C_{n n}^{-1} G_{\phi}+\eta L^{T} L\right) \vec{\Psi}_{2}=\vec{\Psi}_{1}, \\
\left(H_{a}^{T} \tilde{W} H_{a}+\alpha I\right) \vec{\Psi}_{4}=\vec{\Psi}_{3} .
\end{gathered}
$$

The matrix of the first equation is singular because that both $G_{\phi}^{T} C_{n n}^{-1} G_{\phi}$ and $L^{T} L$ have piston in their null space. In order to regularize this equation we can act the 
same way as above with $C_{n n}$-matrix. Piston removal results in the pseudo-inverse in the form

$$
\left(G_{\phi}^{T} C_{n n}^{-1} G_{\phi}+\eta L^{T} L\right)^{\dagger}=\left(G_{\phi}^{T} C_{n n}^{-1} G_{\phi}+\eta L^{T} L+Z Z^{T}\right)^{-1}
$$

where piston projector $Z$ is defined as

$$
Z_{i j}=\left\{\begin{array}{l}
1, \text { if phase point } i \text { is the reference point of screen } j \\
0, \text { otherwise }
\end{array}\right.
$$

The action of such a pseudo-inverse on a vector results in the phase estimate with some arbitrary but finite piston that does not affect the reconstructor performance. Point removal can be done the following way:

$$
\left(G_{\phi}^{T} C_{n n}^{-1} G_{\phi}+\eta L^{T} L\right)^{\dagger}=\left[\left(P_{p r}^{\prime}\right)^{T}\left(G_{\phi}^{T} C_{n n}^{-1} G_{\phi}+\eta L^{T} L\right)\left(P_{p r}^{\prime}\right)\right]^{-1}
$$

where $P_{p r}^{\prime}$ is the point removal projector (3.20) with its zero row removed. Action of this matrix results in the estimate in the form of relative phase referenced to some point. To return to the absolute phase, additional zero should be inserted to the reference point position, which again gives phase estimate with some finite piston present.

Independently of the way regularization of the systems (4.15) and (4.16) is done, their matrices are symmetric and have "sparse + low-rank" form. The important implication of it is that the matrix-vector product with such matrices involved requires only $\mathscr{O}(N)$ operations, so there is the possibility to find the algorithms for solving these equations on-line that are faster than the direct multiplication by the matrix inverse.

Two effective ways of solving these equation systems can be considered.

- Choleski factorization of the sparse system matrix in the form

$$
A=R^{T} R
$$

where $R$ is an upper triangular matrix, which is also sparse, if matrix $A$ is. Special reordering, such as Symmetric Approximate Minimum Degree [44], of 
matrix elements prior to factorization can even somewhat increase the $R$-matrix sparsity. With factorization (4.20) that can be pre-computed off-line, the on-line equation system solution turns into sequential solving the two simple equation systems

$$
R^{T} \vec{y}=\vec{b}, R \vec{x}=\vec{y}
$$

with triangular matrices, which can be effectively done by back-substitution. The complexity of this process is entirely determined by the number of nonzero elements in the Choleski factor $R$. Practical computations show that due to the specific structure of matrices involved in Eqs. (4.15), (4.16) the number of nonzero elements in their Choleski factors is always larger than that of the initial matrix. This difference is almost negligible in the case of SCAO but the block-structured matrices of MCAO system normally produce the Choleski factor, which is almost full. Thus, other methods for the MCAO control are needed.

- The iterative methods are another alternative for effective solving the equations with large sparse matrices. By their nature, all iterative methods involve only matrix-vector multiplications [67], which in the case of sparse matrices can be done very fast. On the other hand, the overall complexity of the iterative solver depends on the number of iterations necessary to converge to the solution with prescribed accuracy. The convergence rate does not depend on the matrix size, which is very favorable for large matrices, but only on the matrix condition number [68]. Unfortunately, the matrices to be inverted in MCAO control are normally ill-conditioned, which makes the direct application of iterative methods ineffective. The cure for this problem is the use of pre-conditioning of the equation system, that is finding some invertible pre-conditioner matrix $C$, such that the equivalent equation system

$$
C^{-1} A x=C^{-1} b
$$


is better conditioned. Multi-grid algorithms is another very effective method to iteratively solve ill-conditioned equation systems. The low complexity multi-grid preconditioned iterative solvers for MCAO, whose brief description is given in Appendix A, have been proposed by C. Vogel et. al. [43, 44, 45] and used to achieve nearly $\mathscr{O}(N)$ complexity wave front reconstruction.

The conclusion of this section is that fast control algorithms for large-scale MCAO based on the minimum variance approach can be created. Moreover, these algorithms were tested through numerous simulations $[47,48]$ and proved to be the good candidates for use in new $\mathrm{AO}$ systems both for their low computational complexity and good phase error rejection. Additional study, however, is necessary to resolve the issues of these algorithms operation on real systems, such as closed-loop stability and sensitivity to the system errors (robustness). The next sections address these problems.

\subsection{Closed-loop operation.}

\section{Idea of the Pseudo Open Loop MV controller.}

An important assumption made in the course of the MV reconstructor derivation is the so called "open-loop" operation, when the direct WFS measurement, $\vec{s}^{o l}$, of the turbulence phase is available. In the real AO systems the direct phase measurement is unavailable because of the insufficient dynamic range of the WFS. Instead, the "closedloop" operation is normally used. As shown on both Fig. 2.1 and 2.3, deformable mirrors precede the wave front sensors, thus the phase residual from partially compensated wave front rather than full phase error is measured. This implies that, when operating in closed loop, we do not have information about the actual turbulence profile. Instead, only the vector $\vec{s}_{t}^{c l}$ of closed-loop slopes produced by uncompensated part of the turbulence profile is available. We want to use this information to find the 
update $\triangle \hat{\vec{\phi}}_{t}$ to the phase profile estimate $\hat{\vec{\phi}}_{t-1}$ made on the previous step such that, according to the minimum variance philosophy,

$$
\triangle \hat{\vec{\phi}}_{t}=\hat{E}^{c l} \vec{s}_{t}^{c l}=\arg \min _{\triangle \vec{\phi}}\left(\left\|\vec{s}_{t}^{c l}-G_{\phi} \triangle \vec{\phi}\right\|_{C_{n n}^{-1}}^{2}+\|\triangle \vec{\phi}\|_{C_{\triangle \triangle}^{-1}}^{2}\right) .
$$

Note that the correct regularization term now is $C_{\triangle \triangle}^{-1}$, the inverse covariance of the turbulence residuals after DM compensation, but this quantity does not have a tractable analytical expression. On the other hand, trying to replace $C_{\triangle \triangle}^{-1}$ with $C_{\phi \phi}^{-1}$ proves to be unreasonably crude approximation. Moreover, it normally causes instability [46]. A simple possible cure was proposed in [47] and is called Pseudo Open-Loop Control (POLC). The idea of POLC is the following:

1) Recover partially the open-loop slope data $\vec{s}_{t}^{p o l}$ from the closed-loop slopes $\vec{s}_{t}^{c l}$ and actuator command vector $\vec{a}_{t}$ computed on each step of reconstruction process.

2) Run open loop reconstruction using the recovered open loop slope data as an input:

$$
\vec{s}_{t}^{p o l}=\vec{s}_{t}^{c l}+G_{a} \vec{a}_{t}
$$

where $G_{a}$ is the DM-to-WFS influence matrix.

That $\vec{s}_{t}^{p o l}$ is only an approximation to the actual open-loop slopes vector $\vec{s}_{t}^{o l}$ is obvious because quite a few factors are not taken into account. These factors are:

1) Noise, miscalibration and misalignments in wave front sensors causing uncertainties in wave front slopes measurements.

2) Exact action of the actuator command on the deformable mirrors because of noise and miscalibration in electrical and mechanical circuits transferring the actuator command signal to DMs.

3) Exact action of DMs on the whole $\mathrm{AO}$ system because of misalignments in its components. 
Nevertheless, the impact of all uncertainties mentioned is expected to be reasonably small for the recovered vector $\vec{s}_{t}^{\text {pol }}$ to represent the open loop data quite correctly. This suggests that $C_{\phi \phi}^{-1}$ is nearly the right regularization term in the Pseudo Open Loop minimization problem:

$$
\hat{\vec{u}}_{t}=\arg \min _{\vec{u}}\left\{\left\|\vec{s}^{p o l}-G_{\phi} \vec{u}\right\|_{C_{n n}^{-1}}^{2}+\|\vec{u}\|_{C_{\phi \phi}^{-1}}^{2}\right\},
$$

where $\vec{u}_{t}=\triangle \vec{\phi}_{t}+\hat{\vec{\phi}}_{t}$, i.e. the sum of closed loop turbulence correction and a turbulence estimate. Solving Eq.(4.25) for the optimal vector $\triangle \hat{\vec{\phi}}_{t}$ yields

$$
\triangle \hat{\vec{\phi}}_{t}=\hat{E}^{o l}\left(\vec{s}_{t}^{c l}+G_{a} \vec{a}_{t}\right)-\hat{\vec{\phi}}_{t}
$$

We next investigate robustness of this scheme, i.e. its stability and performance deterioration with respect to the previously mentioned discrepancies between theoretical system model and the real system.

\subsection{Pseudo Open Loop Control robustness analysis}

In the previous section we have shown that the performance of POLC and its very ability to work in closed-loop depends on the sensitivity of this algorithm to numerous system errors and uncertainties. Possible error factors affecting the system performance are:

1) Misalignments such as shift, rotation and tilt in position of wave front sensors as well as the mismagnification in their conjugation optics. These errors will induce changes in the phase-to-WFS influence matrix $\hat{G}_{\phi}$ assumed when computing the reconstructor and result in the real system matrix $G_{\phi}$ representing misaligned wave front sensors. 
2) Wave front sensor calibration errors to result in some uncontrolled time invariant contribution $\delta \vec{s}$ to the slopes.

3) Geometrical misalignments such as shift, rotation, tilt and mismagnification in the deformable mirrors. This will induce changes in the DM-to-WFS influence matrix $\hat{G}_{a}$ assumed for phase reconstruction and result in the real system matrix $G_{a}$. It is important to note that since $G_{a}$ is also related to WFS all misalignments in wave front sensors affect this matrix as well. Deformable mirror misalignments affect also the DM-to-aperture interaction matrix $H_{a}$.

4) Calibration and alignment errors in deformable mirrors mechanics, miscalibration and noise in their electrical circuits resulting in the uncontrolled contribution $\delta \vec{a}+\vec{n}_{a}$ to the actuator command $\vec{a}_{t}$, where $\delta \vec{a}$ is the unknown time invariant contribution to the actuator command, $\vec{n}_{a}$ the actuator commands noise.

The combined effect of the turbulence and actuator commands on the pseudo openloop WFS measurement and the residual phase can be written as

$$
\begin{gathered}
\vec{s}_{t}^{p o l}=G_{\phi} \vec{\phi}_{t}-G_{a}\left(\vec{a}_{t}+\delta \vec{a}+\vec{n}_{a}\right)+\delta \vec{s}+\vec{n}_{t}, \\
\vec{\epsilon}_{t}=H_{\phi} \vec{\phi}-H_{a}\left(\vec{a}_{t}+\delta \vec{a}+\vec{n}_{a}\right),
\end{gathered}
$$

The corrupted $G$ - and $H$-matrices appearing in the above equations are computed in a straightforward way with the aid of grid points' coordinate transformations intended to account for misalignments. Appendix B contains a description of the effective numerical procedure to compute the elements of $G$-matrices.

The $z$-domain block diagram of the AO system with Pseudo Open Loop Controller and all error factors taken into account is shown on Fig. 4.3. Here $c(z)$ is a servo compensator filter connected in the negative feedback loop and responsible for the system temporal behavior. 


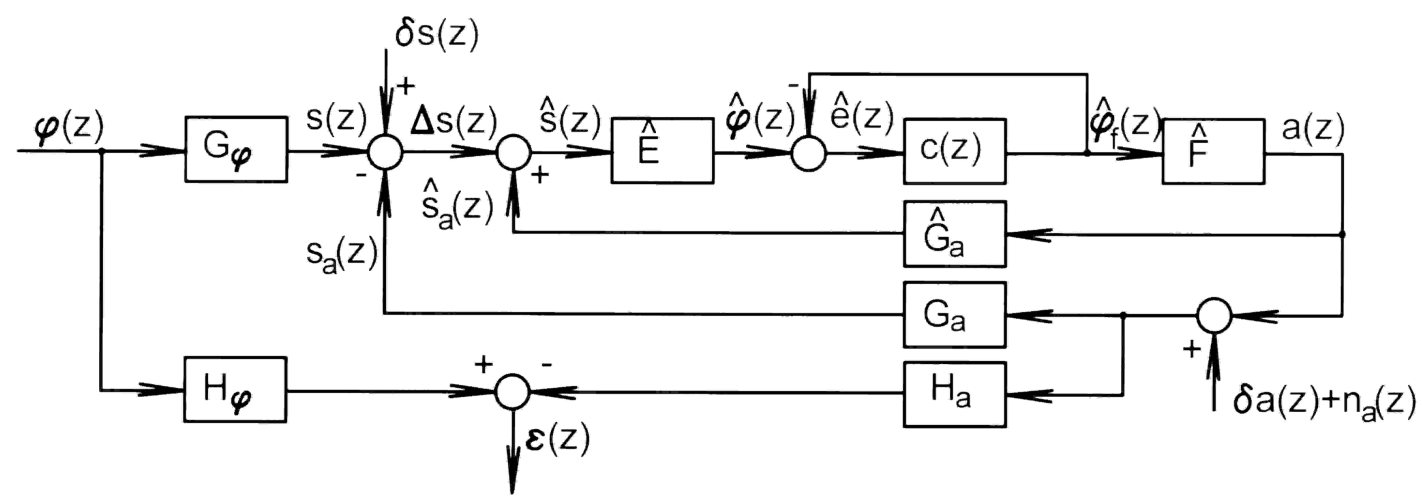

Figure 4.3: $z$-domain block diagrams of non-ideal MCAO system driven by POLC.

Note that the system depicted on Fig. (4.3) should be viewed as consisting of two independent parts:

1) boxes with hatted transfer matrices represent the "reconstructor part", which involves only information about the idealized system and is "unaware of" the errors present;

2) all the remaining blocks represent the model for the "real" AO system and involve all the information about various error factors.

It is this interplay between the "ideal" reconstructor and a "real" system that enables one to reveal and estimate the possible stability and performance degradation that may occur in practice. It is easy to find the expression for $T(z)$ :

$$
T(z)=g(z)\left[I+g(z)\left(I-\hat{E} G_{a} \hat{F}\right)\right]^{-1} \hat{E}
$$

and for the closed-loop transfer matrix function $H_{C L}(z)$ :

$$
\triangle \vec{s}(z)=H_{C L}(z) \vec{\phi}(z), H_{C L}=G_{a} \widehat{F} Q(z) \hat{E} G_{\phi},
$$

where

$$
Q(z)=g(z)[I+g(z) M]^{-1}
$$




$$
M=I+\hat{E}\left(G_{a}-\hat{G}_{a}\right) \hat{F}
$$

Eqs. (4.30)-(4.32) are the basis for stability analysis of the POLC system. The system is stable, if all poles of $H_{C L}(z)$ lie inside the unit circle on the complex z-plane. It is obvious from Eq. (4.30) that these poles are the same as those of $Q(z)$. Note that, as it follows from Eq. (4.32), all pole movement caused by the system errors is driven only by the DM-to-WFS matrix $G_{a}-\hat{G}_{a}$, which comprises information about misalignments in both WFSs and DMs. It is not difficult to see using the spectral representation of Q-matrix (see Appendix C) that poles of $Q(z)$ are those of the scalar functions

$$
c_{i}(z)=\frac{c(z)}{1+\lambda_{i} c(z)},
$$

where $\lambda_{i}$ are the eigenvalues of M-matrix. Eq. (4.33) is the basic tool for the theoretical stability analysis of MCAO system. The important fact about stability of the POLC is that in the perfectly aligned case the $M$-matrix has only one and zero eigenvalues and the system temporal behavior is completely determined by poles and zeros of the servo compensator closed-loop transfer function $c(z) /(1+c(z))$.

Remarkably, the stability pattern of the POLC depends solely on the misaligned DM-to-WFS matrix $G_{a}$. On the other hand, provided the system is stable, its overall performance will depend on all error factors involved. To estimate the performance degradation due to the various errors mentioned previously, Monte-Carlo simulations were performed. Residual phase errors and average Strehl ratios were used as performance metrics. The results of these simulations are presented in the next section.

\subsection{Simulation results}

The simulation results presented below are obtained for a representative case of the system similar to the Gemini-South $8 \mathrm{~m}$ telescope MCAO system [59]. Its basic setup 
is:

- Primary mirror diameter is 8 meters, no central obscuration is considered.

- 5 mesospheric (altitude 90 kilometers) LGS Shack-Hartman wave front sensors, 4 single-aperture NGS sensors for global tilt measutemnts, whose parameters are given in Table 4.1. LGS wave front sensors subaperture arrangement is shown on Fig. (4.4). Note that the NGS WFS contain only single subaperture that inscribes the system entrance pupil.

\begin{tabular}{|c|c|c|}
\hline & LGS WFS & NGS WFS \\
\hline Number of sensors & 5 & 4 \\
\hline Subaperture grid size & $16 \times 16$ & $1 \times 1$ \\
\hline Subaperture dimensions, m & 0.5 & 8.0 \\
\hline Total number of subapertures in each sensor & 224 & 1 \\
\hline
\end{tabular}

Table 4.1: Wave front sensors of the Gemini-South $8 \mathrm{~m}$ telescope MCAO system.

- LGS \& NGS 90/60 arcsec arrangement and 60 arcsec scientific field of view as shown on Fig. (4.5).

- Noise equivalent angles for LGS and NGS wave front sensors are fixed to be around 30 mas and 3 mas, respectively.

- 3 deformable mirrors, whose parameters are given in Table 4.2. Only the actuators within beamprints created by the main aperture projections along scientific directions are kept active. The active actuators arrangement for each DM is shown on Fig. (4.6). Bilinear splines are taken to approximate the DM influence functions.

- Turbulence is represented by 6 phase screens, whose altitudes, strengths and wind velocities have been obtained from 57 layer Cerro Pachon turbulence profile [6] using the technique described in Sec. 3.1. Fried parameter $r_{0}$ is equal to $0.16 \mathrm{~m}$ at $0.5 \mu \mathrm{m}$. 


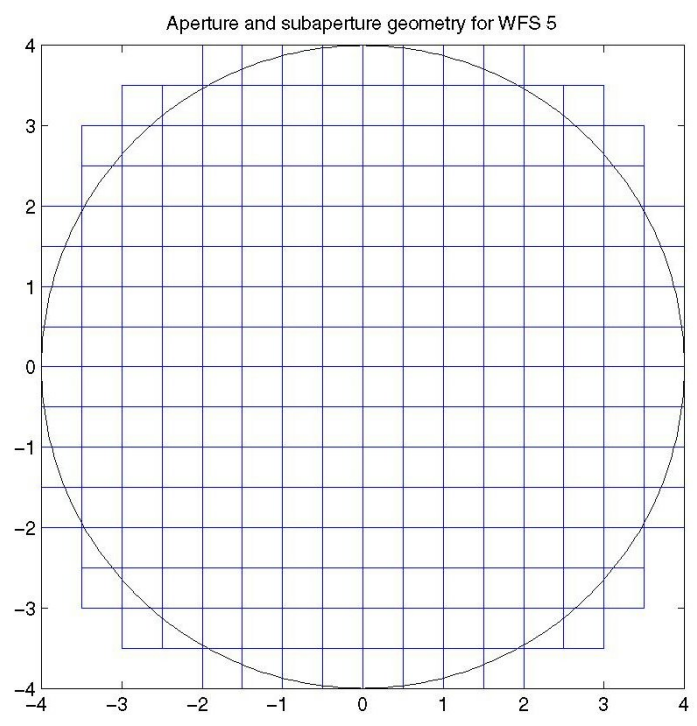

Figure 4.4: Subaperture arrangement in LGS wave front sensor. 224 active subapertures fill the main aperture.

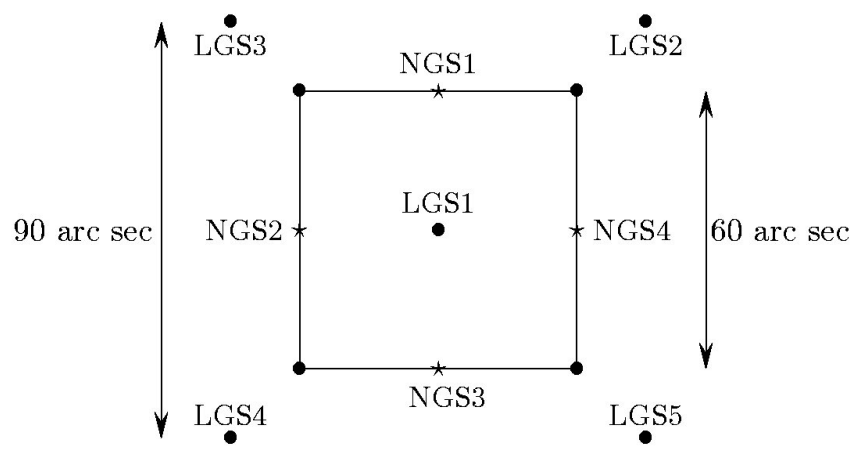

Figure 4.5: Representative Gemini-South $8 \mathrm{~m}$ telescope MCAO system guide stars arrangement and scientific field of view $(\mathrm{ScFoV})$. 

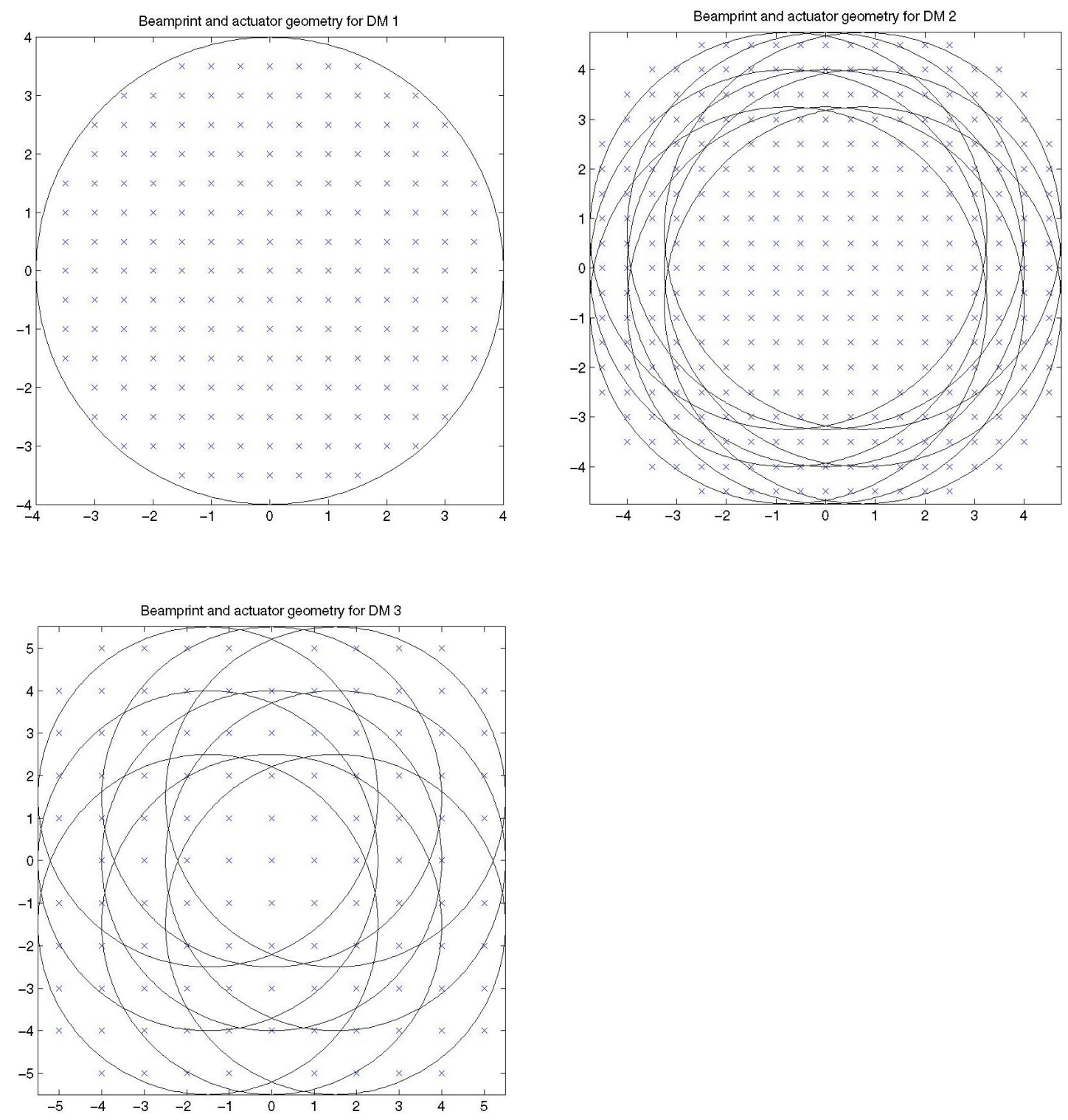

Figure 4.6: Deformable mirrors actuator grids. Active actuators are shown with crosses. The circles are the main aperture projections onto DMs along the scientific directions. 


\begin{tabular}{|c|ccc|}
\hline Deformable mirror number & 1 & 2 & 3 \\
\hline Conjugation altitude, $\mathrm{km}$ & 0.0000 & 5.1546 & 10.3090 \\
\hline Actuator grid size & 15 & 20 & 12 \\
\hline Total number of actuators & 193 & 329 & 113 \\
\hline Inter-actuator spacing, m & 0.5 & 0.5 & 1.0 \\
\hline
\end{tabular}

Table 4.2: Gemini-South $8 \mathrm{~m}$ telescope MCAO system deformable mirror parameters.

Bilinear splines (3.7) are taken for basis for phase approximation. The phase screens and their simulation and reconstruction phase grids parameters are given in Table 4.3. The reconstruction phase grids geometries for all phase screens are shown on Fig. 4.7. Only phase points that contribute to the imaging and WFS measurements are kept to minimize computational burden. These are the points within the beamprint, i.e. the union of main aperture projections onto a phase screen along the scientific and guide star directions, shown as circles on Fig. 4.7. Note that the resolution of simulation phase grids is taken to be 8 times higher than the resolution of the densest DM actuator grids.

- System's temporal behavior in closed loop is driven by the servo compensator, whose transfer function is

$$
c(z)=\frac{\delta}{z^{2}-\alpha z-\beta}
$$

where the parameters are taken to be $\alpha=\beta=0.495$ and $\delta=0.5$ to represent the leaky integrator with 2-frame latency. The closed-loop $\left|c\left(\nu / \nu_{s}\right) /\left(1+c\left(\nu / \nu_{s}\right)\right)\right|^{2}$ and error rejection $\left|1 /\left(1+c\left(\nu / \nu_{s}\right)\right)\right|^{2}$ power spectra of this integrator are shown on Fig. 4.8. With the sampling rate taken equal to $\nu_{s}=800 \mathrm{~Hz}$, the $-3 \mathrm{~dB}$ closed-loop bandwidth of the compensator is equal to $88 \mathrm{~Hz}$ and the rejection bandwidth is $32 \mathrm{~Hz}$, which corresponds to the ability to work stable for average wind speeds up to $\simeq 12 \mathrm{~m} / \mathrm{s}$ for $\lambda=0.55 \mu \mathrm{m}$. 

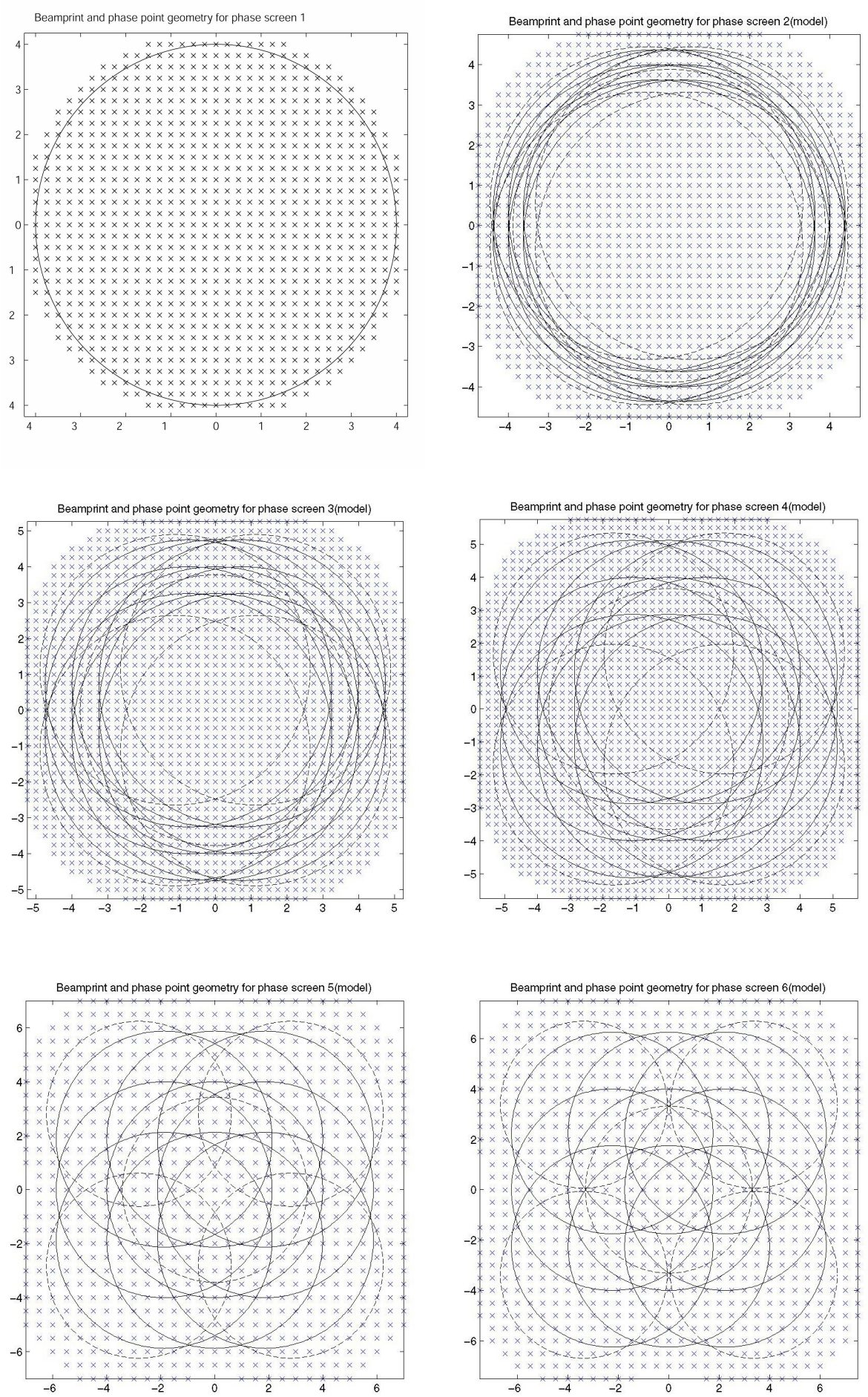

Figure 4.7: Reconstruction phase grids on the six phase screens, whose parameters are given in Table 4.3. Grid points positions are shown with crosses. The circles are the main aperture projections onto the phase screens along the scientific and guide stars directions. 


\begin{tabular}{|c|cccccc|}
\hline Phase screen number & 1 & 2 & 3 & 4 & 5 & 6 \\
\hline Altitude, $\mathrm{km}$ & 0.0000 & 2.5773 & 5.1546 & 7.7320 & 12.8870 & 15.4640 \\
\hline Weight & 0.6523 & 0.1723 & 0.0551 & 0.0248 & 0.0736 & 0.0219 \\
\hline Wind speed vector, m/sec & $(5,0)$ & $(13,0)$ & $(20,0)$ & $(30,0)$ & $(20,0)$ & $(10,0)$ \\
\hline Reconstruction grid size & 33 & 39 & 43 & 47 & 29 & 31 \\
\hline Reconstruction grid spacing, $\mathrm{m}$ & 0.5 & 0.5 & 0.5 & 0.5 & 1.0 & 1.0 \\
\hline Reconstruction grid points \# & 1149 & 1405 & 1629 & 1933 & 773 & 877 \\
\hline Simulation grid size & 133 & 145 & 157 & 169 & 193 & 205 \\
\hline Simulation grid spacing, $\mathrm{m}$ & 0.0625 & 0.0625 & 0.0625 & 0.0625 & 0.0625 & 0.0625 \\
\hline Simulation grid points \# & 14197 & 17533 & 21157 & 25069 & 33717 & 38437 \\
\hline
\end{tabular}

Table 4.3: Six-layer turbulence model used in the simulation of the Gemini-South $8 \mathrm{~m}$ telescope MCAO system.

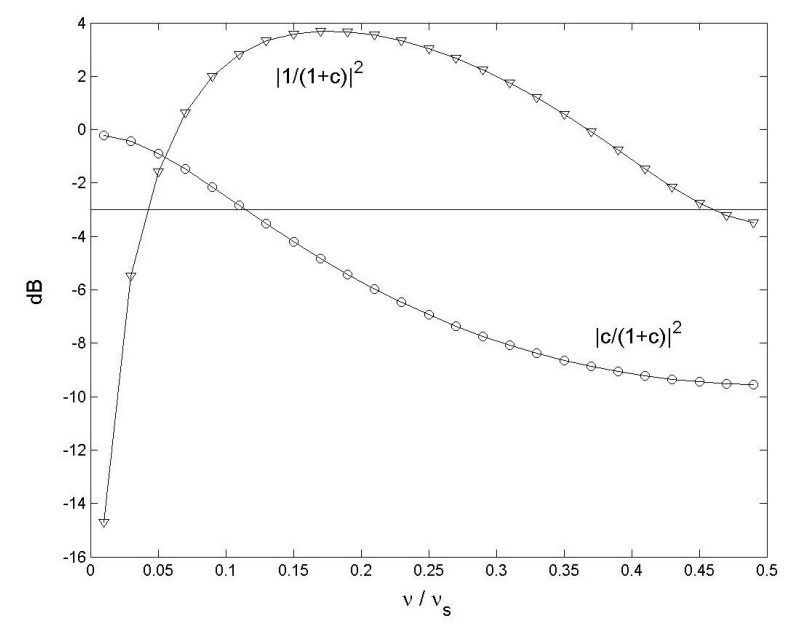

Figure 4.8: Closed-loop (circles) and rejection (triangles) power spectra of the servo compensator used in the system. 
Performance of the system was analyzed in two ways:

1. Stability behavior was investigated based on Eqs. (4.30)-(4.33). Position and evolution of closed loop transfer matrix poles with respect to various kinds and amounts of misalignment were computed. Approximate stability margins were found. The stability results for POLC were compared to those of the Least Squares Control (LSC) described in Sec. 3.6.1. Monte-Carlo simulations were performed to confirm the stability/instability conclusions made based on the eigenvalue analysis.

2. Provided the AO system was stable, its performance degradation was analyzed by running Monte-Carlo simulations as described in Sec. 3.7 and with sparse MV reconstructor applied as described in Sec. 4.1. Input phase screens with Kolmogorov statistics were generated via Fourier domain method with tilt correction [41]. Wind shift was modeled via Fourier shift theorem (3.59). System performance metrics computed are the piston-removed aperture phase residuals as a function of time and long-exposure Strehl ratios.

Of course, it is hardly possible to analyze the effect of all combinations of error factors on system performance. Presented below are certain benchmark results illustrating the effect of each error factor isolated from the others, though the computation approach used in this work is general enough to consider any combination of system errors. Following errors were considered:

1) shifts, rotations and mismagnifications in deformable mirrors and wave front sensors;

2) tilts in DMs and WFSs to represent a possible kind of constant miscalibration errors $\delta \vec{s}$ and $\delta \vec{a}$; 
3) noise in DM command vector $\vec{a}$ (see Eqs. (4.27), (4.28)).

Note that two last error factors do not affect stability but can deteriorate performance of an $\mathrm{AO}$ system.

\subsubsection{Stability analysis results}

The eigenvalues of the matrix $M$ in Eq. (4.32) and, therefore, poles of closed-loop transfer function were found numerically for the sequences of increasing values of system misalignments. The threshold at which at least one of the poles moves outside the unit circle gives the approximate stability margin for a given type of error. Presented on Fig. 4.9 is the sequence of frames showing the POLC closed-loop transfer function poles evolution in the case of DM shift misalignment increasing from $10 \%$ to $60 \%$ of the inter-actuator spacing. It is seen from the position of the poles with respect to the circle of stability that the system remains stable up to the shifts equal $50 \%$.

The stability margins are presented in Tables 4.4 and 4.5 . It is clearly seen that the POLC is remarkably stable against misalignments in DMs and WFSs. It can tolerate shifts as large as $50 \%$ of subaperture size or inter-actuator spacing and 4 deg rotational misregistration. Moreover, it significantly outperforms in this respect LSC, which is also confirmed by Monte-Carlo simulations.

\subsubsection{Performance analysis results}

Following are the examples of performance deterioration caused by the system errors, which are small enough to keep the system stable. Table 4.6 gives the long exposure Strehl ratios for different types and levels of system errors. Figs. 4.10-4.13 show the plots of the aperture residual phase variance (3.42) time histories for shift, rotation, mismagnification and tilt misalignment errors, respectively, in both DMs and WFSs. 

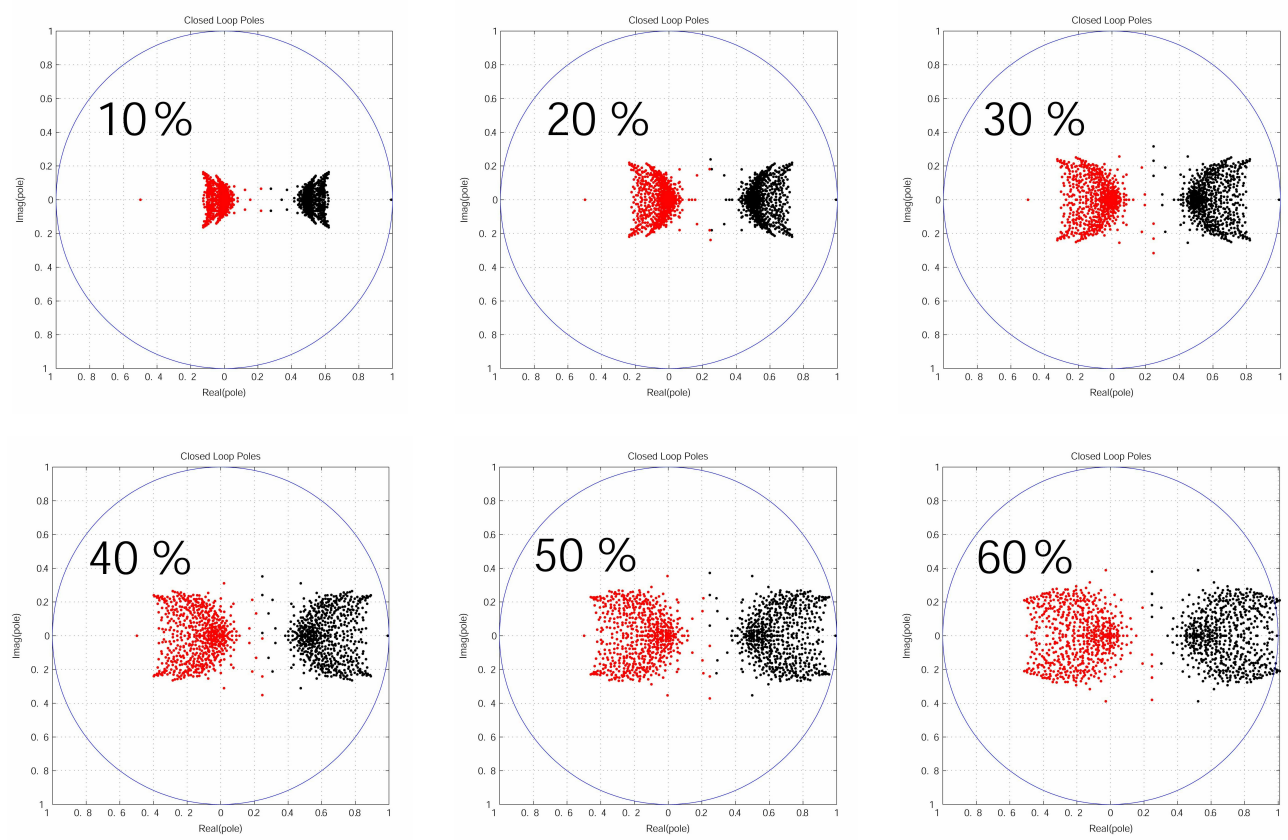

Figure 4.9: Transfer function poles evolution as a function of the DMs shift misalignment value. Each frame gives positions of the closed-loop system poles for different degree of misalignment. All three DMs are shifted simultaneously from their perfect alignment position in directions that make a 120 deg angle with one another. 


\begin{tabular}{|c|c|c|c|}
\hline Mirror misaligned & Shift, $\%$ & Rotation, rad & Mismagnification \\
\hline 1st & $10 / 50$ & $0.07 / 0.07$ & - \\
\hline 2nd & $30 / 50$ & $0.03 / 0.07$ & - \\
\hline 3rd & $30 / 50$ & $0.07 / 0.07$ & - \\
\hline All three & $30 / 50$ & $0.03 / 0.07$ & $1.03 / 1.15$ \\
\hline
\end{tabular}

Table 4.4: Approximate stability margins for the various misalignments in DMs for LSC (first number) and for POLC (second number). Single-mirror shifts are done in $45 \mathrm{deg}$ direction, i.e. $\mathrm{x}$-component of the shift is equal to the $\mathrm{y}$-component. Three-mirror shifts are done such that the shift directions of the mirrors make an angle 120 deg with one another. Three-mirror rotations are done such that the rotation directions of the mirrors are opposite to one another. Mismagnification is the same for each DM.

\begin{tabular}{|c|c|c|}
\hline Shift in all WFSs, \% & Rotation in all WFSs, rad & Mismagnification in all WFSs \\
\hline $50 / 50$ & $0.07 / 0.07$ & $1.10 / 1.11$ \\
\hline
\end{tabular}

Table 4.5: Approximate stability margins for misalignments in WFSs. Shift directions in WFSs make a 120 deg angle with one another. WFS rotation is done clockwise in all sensors. WFS mismagnification is the same for each sensor. 
Fig. 4.14 shows the residual phase variance time histories for the different levels of additive noise in the DM commands. The error history graph simulated for the perfectly aligned system is added in all figures for comparison. The cases when the system runs unstable in Monte-Carlo simulations confirm the same stability/instability conclusions drawn from the transfer function spectral analysis in the sense that the POLC runs stable or unstable for the values of system errors predicted by the spectral analysis. Another observation is that the performance of the MCAO system driven by POLC deteriorates quite slowly with the increase of system errors. For instance, it is virtually unaffected by the $10 \%$ shift, 0.01 rad rotation or $1 \%$ overmagnification in DMs or WFSs. The same can be said for 25 mas tilt in each DM or 2.5 mas tilt in each WFS or $1 \%$ additive noise in actuator command. Relatively high sensitivity to WFS tilt is mostly due to the tip-tilt WFSs. As it is clear from Fig. 4.13 (lower panel), performance of the system with only LGS WFSs tilted is very close to that of ideal system. Necessity for the tip-tilt correction to be done more carefully is in agreement with the fact that tilt is the most significant fraction of the entire phase error to be corrected.

\subsection{Robustness analysis conclusions.}

Stability and performance analysis carried out in this chapter clearly show the superior robustness of POLC and its capability of running adaptive optics system in closed loop despite the initial assumption about the precise knowledge of the current system state for POLC to work [48]. Favorable performance behavior is undoubtedly owing to the use of a priori information about turbulence statistics inherent in minimum variance approach, which helps to compensate the information loss in the form of system errors. The theoretical stability margins and tolerable system error values obtained can be used as benchmarks in the course of designing new MCAO systems. 

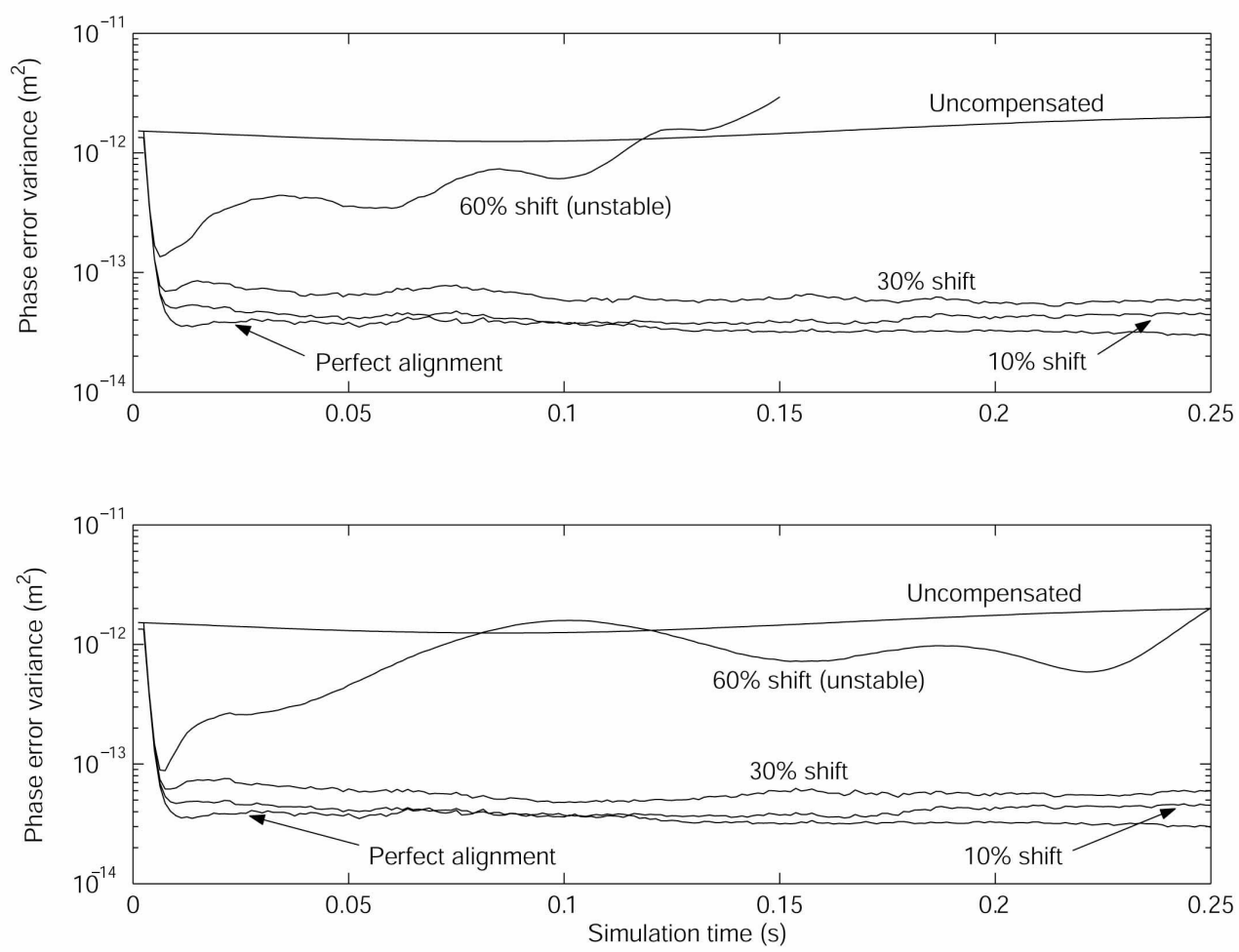

Figure 4.10: Wave front phase error variance time histories for a number of shift misalignments levels in DMs (upper panel) and WFSs (lower panel). DM shift directions make a 120 deg angle with one another. WFS shift directions make a 45 deg angle with one another. 

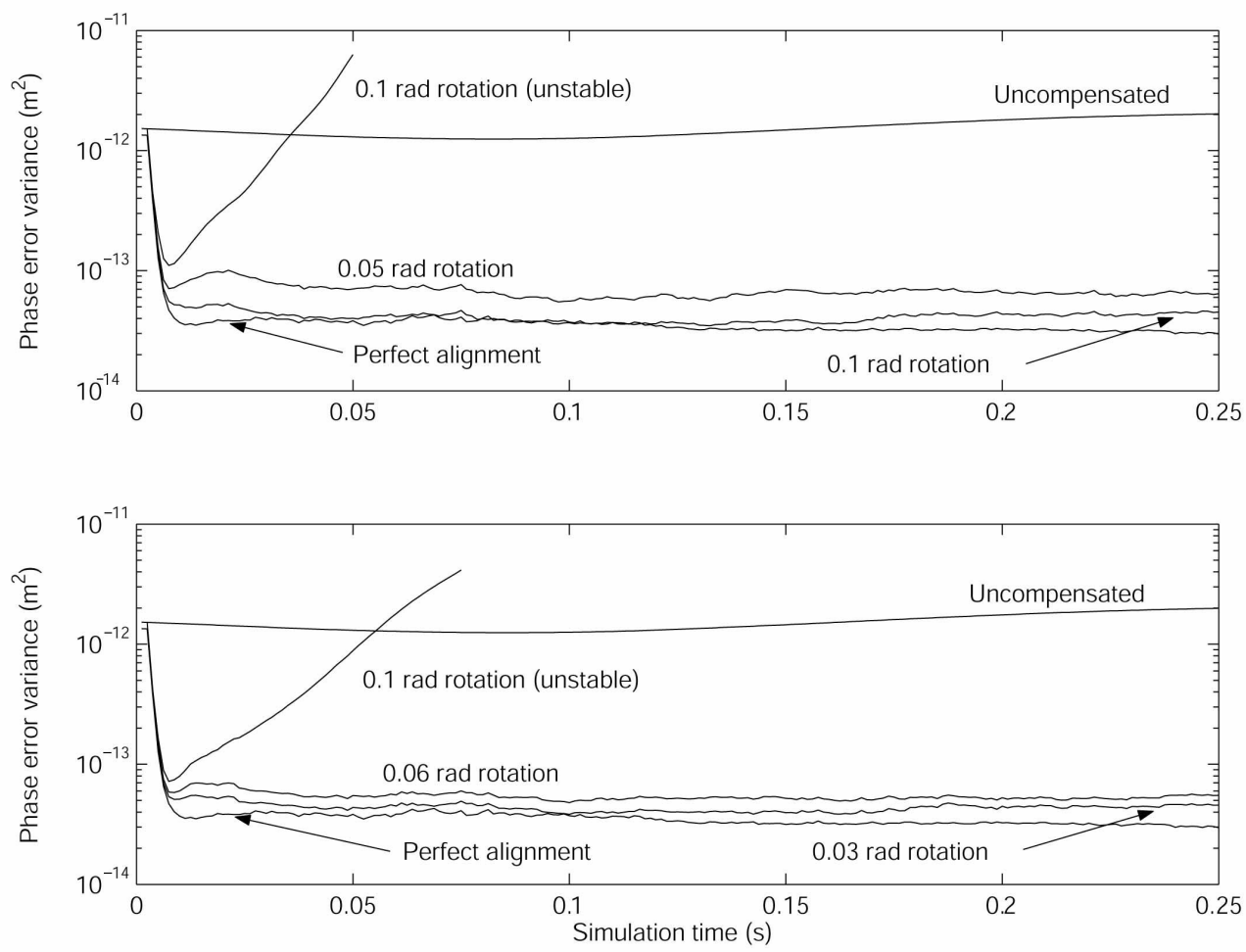

Figure 4.11: Wave front phase error time histories for a number of rotation misalignment levels in DMs (upper panel) and WFSs (lower panel). Rotations in each DM are opposite to one another. All WFSs are rotated clockwise. 

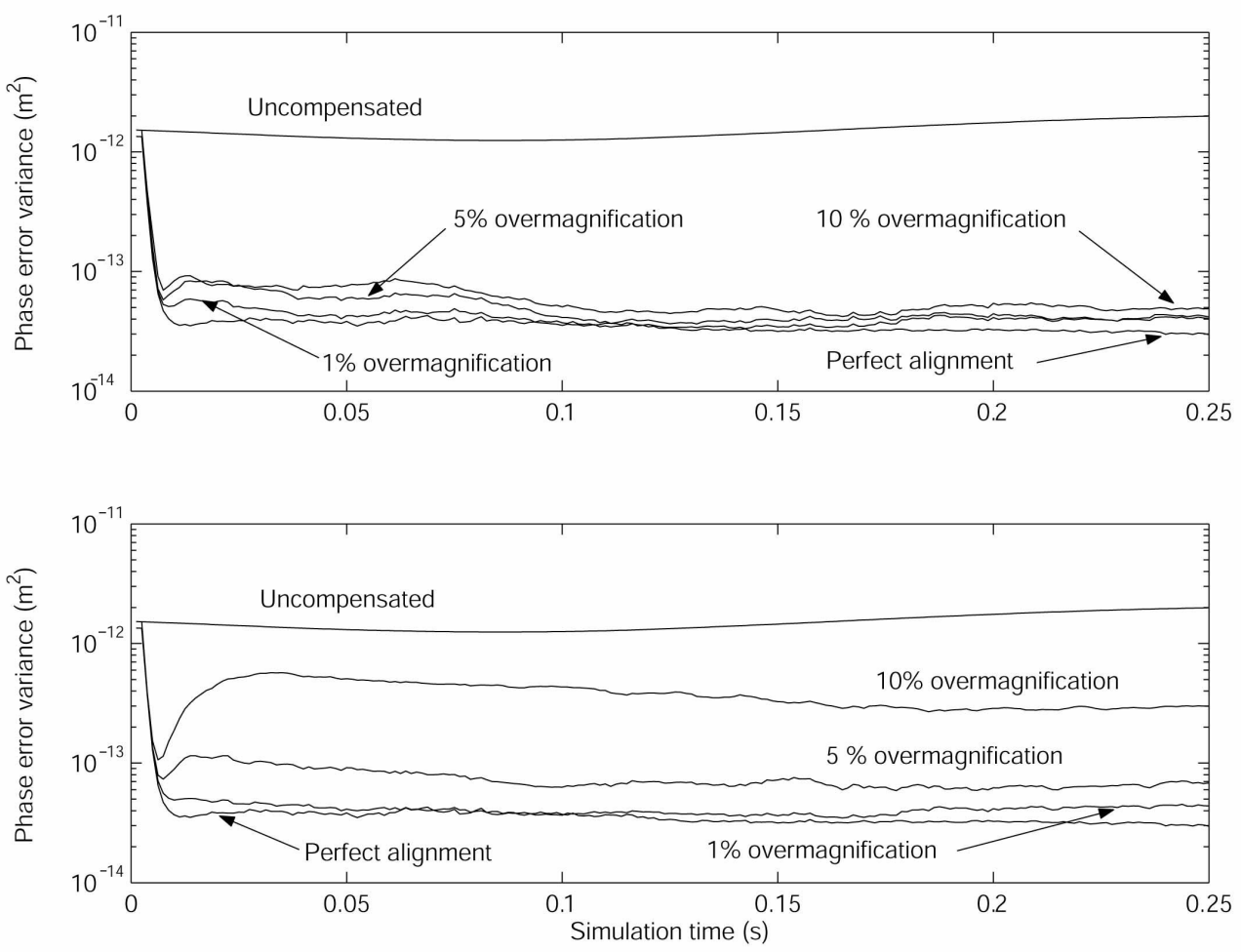

Figure 4.12: Wave front phase error time histories for different levels of mismagnification in DMs (upper panel) and WFSs (lower panel). Mismagnifications are set equal for each DM or WFS. 

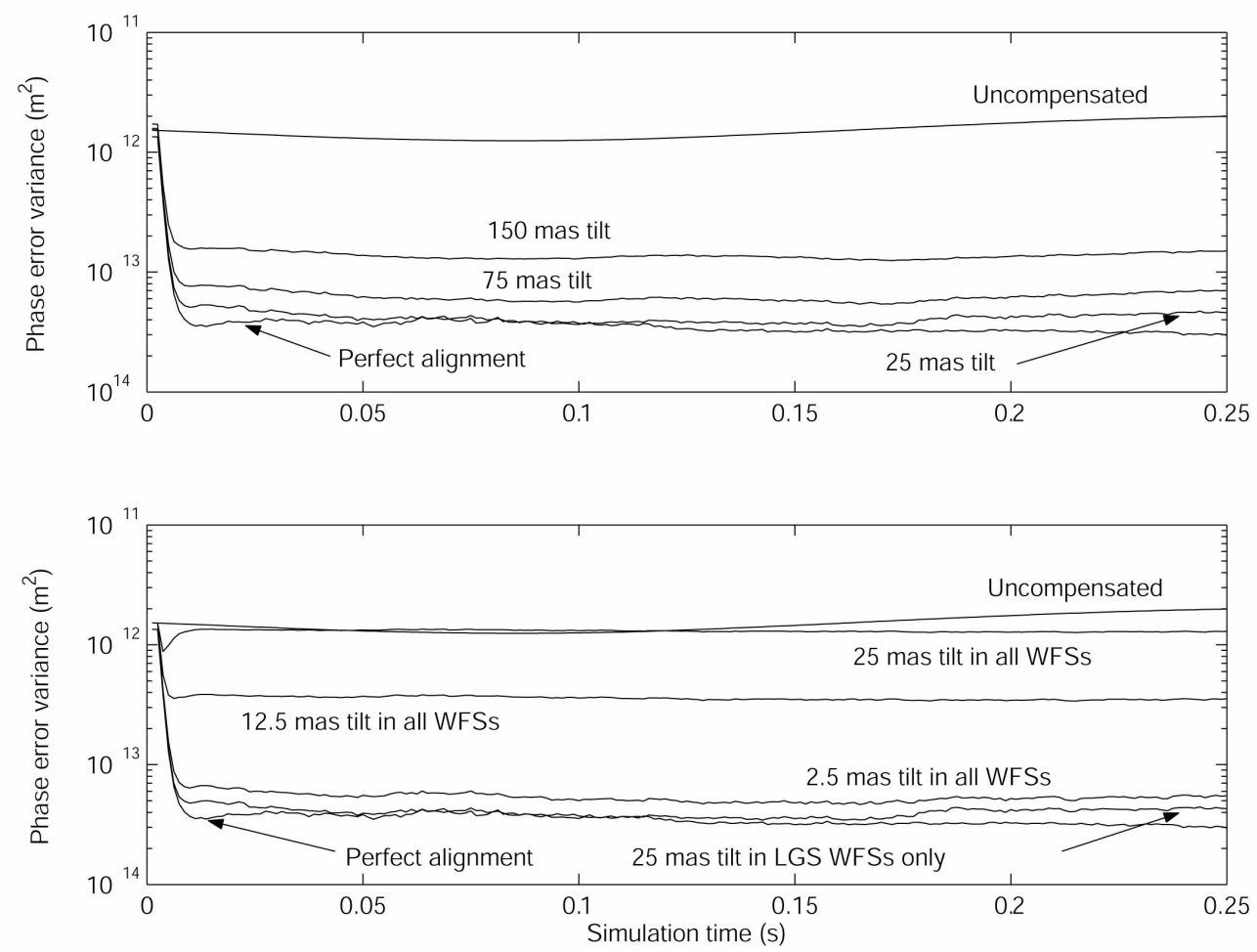

Figure 4.13: Wave front phase error time histories for different tilt levels in DMs (upper panel) and WFSs (lower panel). Tilt directions in each DM make a 120 deg angle with one another. Tilt directions in each WFS make a 45 deg angle with one another. 


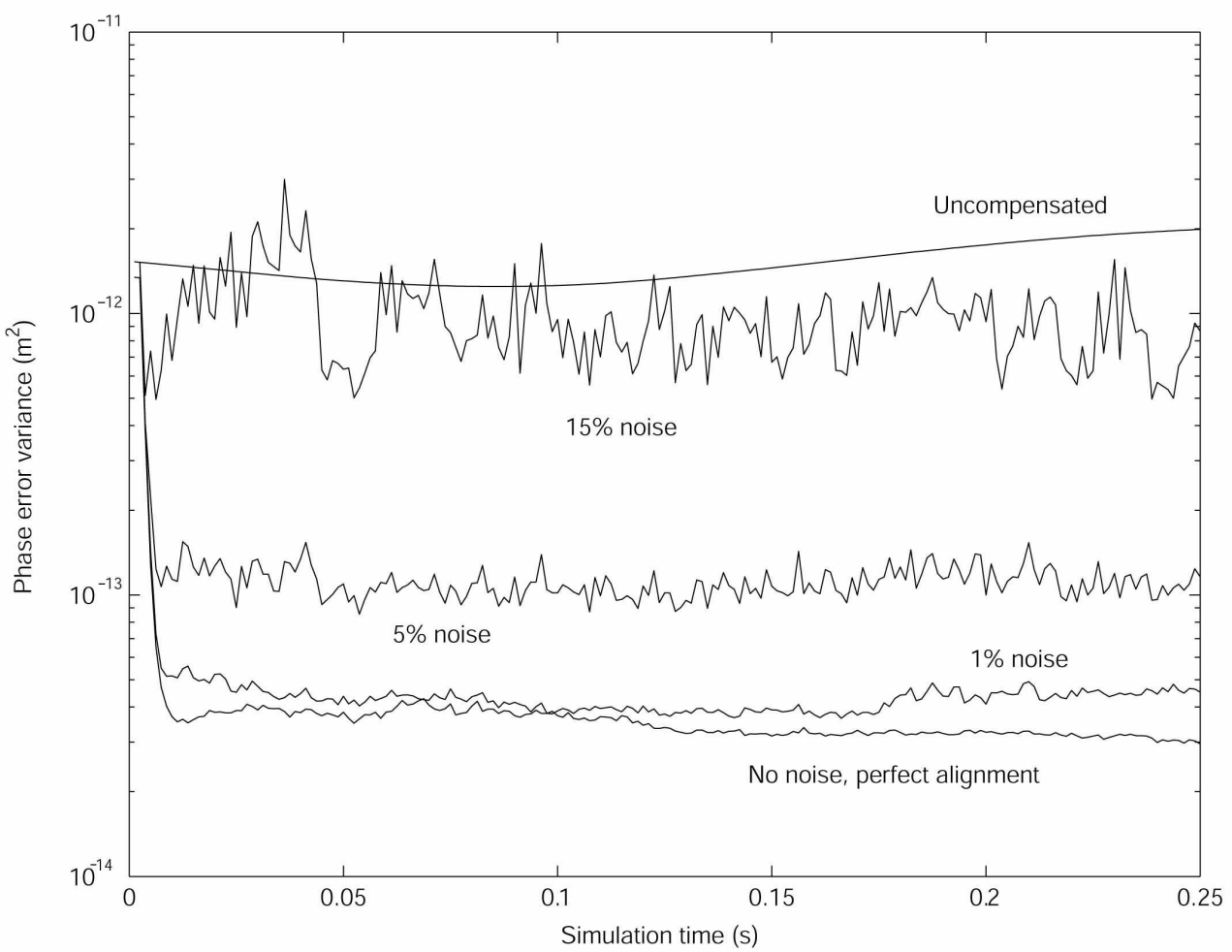

Figure 4.14: Wave front phase error time histories for different levels of additive noise in the DM actuator command vector. White Gaussian noise is assumed. The noise level is given as a fraction of the largest actuator command vector component at each time step. 


\begin{tabular}{|c|c|c|c|}
\hline System error type & $\mathrm{J}$ band & $\mathrm{H}$ band & $\mathrm{K}$ band \\
\hline Perfect alignment & $.53 \quad .33 \quad .25$ & $\begin{array}{lll}69 & .52 & .43\end{array}$ & $.81 .68 \quad .60$ \\
\hline $10 \%$ shift in all DMs & $.52 \quad .32 \quad .24$ & $.68 \quad .51 .41$ & $.80 \quad .68 \quad .59$ \\
\hline $10 \%$ shift in all WFSs & $.53 \quad .32 \quad .25$ & $\begin{array}{lll}69 & .50 & .43\end{array}$ & $.81 \quad .67 \quad .60$ \\
\hline $30 \%$ shift in all DMs & .47 .27 .18 & $.64 \quad .46 \quad .35$ & $.77 \quad .64 \quad .53$ \\
\hline $30 \%$ shift in all WFSs & .41 .22 .18 & $.59 .41 \quad .33$ & $.74 \quad .59 \quad .49$ \\
\hline $0.01 \mathrm{rad}$ rotation in $\mathrm{DMs}$ & $.53 .33 \quad .24$ & $\begin{array}{lll}.69 & .52 & .42\end{array}$ & $.81 .69 \quad .60$ \\
\hline $0.05 \mathrm{rad}$ rotation in $\mathrm{DMs}$ & $.45 \quad .25 \quad .19$ & $\begin{array}{lll}.62 & .43 & .35\end{array}$ & $\begin{array}{lll}.76 & .61 & .54\end{array}$ \\
\hline $0.03 \mathrm{rad}$ rotation in WFSs & $.50 \quad .32 \quad .21$ & $\begin{array}{lll}67 & .50 & .39\end{array}$ & $.80 \quad .67 \quad .58$ \\
\hline $0.06 \mathrm{rad}$ rotation in WFSs & $.43 \quad .25 .18$ & $.61 .44 \quad 32$ & $.75 \quad .62 \quad .51$ \\
\hline $1 \%$ overmagnification in DMs & $.50 .34 \quad .23$ & $.67 \quad .52 \quad .41$ & $.80 \quad .69 \quad .60$ \\
\hline $5 \%$ overmagnification in DMs & $.45 \quad .29 .17$ & $.60 \quad .45 \quad .35$ & $.75 \quad .65 \quad .54$ \\
\hline $1 \%$ overmagnification in WFSs & $.54 \quad .32 \quad .25$ & $\begin{array}{lll}.70 & .50 & .42\end{array}$ & $.82 \quad .67 \quad .60$ \\
\hline $5 \%$ overmagnification in WFSs & .37 .16 .12 & $.55 \quad .33 \quad .27$ & $\begin{array}{lll}.71 & .52 \quad .40\end{array}$ \\
\hline 25 mas tilt in all DMs & $\begin{array}{lrr}.51 & .33 & .22\end{array}$ & $.68 \quad .52 \quad .34$ & $.80 \quad .69 \quad .58$ \\
\hline 75 mas tilt in all DMs & .46 . 19. & $.63 \quad .34 \quad .24$ & $.77 \quad .53 \quad .42$ \\
\hline 2.5 mas tilt in all WFSs & $\begin{array}{lll}.53 & .31 & .26\end{array}$ & .69.41 & $.81 \quad .63 \quad .55$ \\
\hline 12.5 mas tilt in all WFSs & .37 .14 .13 & $\begin{array}{lll}.56 & .31 & .27\end{array}$ & $.72 \quad .50 \quad .44$ \\
\hline $1 \%$ actuator command noise & $.53 \quad .34 \quad .22$ & $\begin{array}{lll}.69 & .53 & .40\end{array}$ & $\begin{array}{lll}.81 & .69 & .59\end{array}$ \\
\hline $5 \%$ actuator command noise & $\begin{array}{lll}.07 & .06\end{array}$ & .28 .20 .18 & $\begin{array}{lll}.48 & .39\end{array}$ \\
\hline
\end{tabular}

Table 4.6: Average Strehl ratios obtained for various types and degrees of system errors. In each triad first, second and third numbers correspond, respectively, to the center, right side and upper right corner of the field of view. The statistical error in the Strehl ratios computation does not exceed $10 \%$ in all cases. 


\section{CHAPTER 5}

\section{Performance Analysis of Minimum Variance and Kalman Filter Controllers}

This chapter concentrates on the comparison of Minimum Variance (MV) and Kalman Filter (KF) control algorithms. As it has been shown in the previous chapters, the MV algorithm uses a priori information about atmospheric turbulence statistics in the form of covariance matrices, which enables better error rejection in comparison to the Least Squares approach widely used in conventional AO and also improve robustness because the statistical priors tend to fill the information gaps caused by imperfect measurements and system errors. It has also been shown that the zonal MV algorithm can be implemented in sparse form with computational complexity nearly $\mathscr{O}(N)$, where $N$ is a number of system's degrees of freedom. On the other hand, the MV approach has at least two major shortcomings:

- It is non-dynamic, i.e. uses only the current WFS measurement to find the turbulence phase estimate. But, since the atmospheric turbulence is highly temporally correlated, the use of previous measurements, which are also available, could significantly reduce the estimation error.

- In its classical form [52], the MV approach does not have the means for system state temporal prediction in order to compensate for the lag always present in real AO systems. 
These two serious problems motivate investigation into alternative approaches. Having much in common with MV approach, the KF, whose idea was briefly described in Sec. 3.6.3, has the potential to overcome the drawbacks of the MV. KF approach has been considered in conventional single conjugate AO for decades $[53,54,56]$. New promising results have been also obtained for MCAO in the frame of modal approach [57]. The main problem of the KF approach is that in the standard form it has $\mathscr{O}\left(N^{2}\right)$ complexity and its computational structure is not suitable for further complexity reduction, which is crucial for large scale AO. Therefore, it is prudent to evaluate the possible payoff, that is the performance advantage achievable with $\mathrm{KF}$ in comparison to other, computationally less intense approaches. The goal of the work presented in this chapter is to show the performance modelling results for $\mathrm{KF}$ as atmospheric phase distortion estimator for an MCAO system in the frame of a zonal correction approach with direct comparison to the zonal MV estimator. The other goal is to evaluate capabilities of simple turbulence dynamics models in resolving the time delay issue for both $\mathrm{KF}$ and MV. In many ways the results presented below can be considered an MCAO extension of the D. Gavel and D. Wiberg's work [56] on KF for SCAO.

\subsection{Near-Markov turbulence model.}

As it was pointed out in Sec. 3.6.3, in order to use the KF for atmospheric turbulence estimation it is necessary to come up with some linear autoregressive (AR) model approximating turbulence dynamics. On one hand, the form of Kolmogorov (2.4) or von Karman (2.5) turbulence power spectral density is impossible to approximate really well with a low order AR model. On the other hand, higher order models can be computationally prohibitive as the size of state vector $\vec{X}_{t}$ will increase drastically. In our simulations we used the simplest first order $\mathrm{AR}(1)$ model, i.e. only the current

phase $\vec{\phi}_{t}$ is taken for the system's state. Since in such a model the next state value 
depends on the previous one only, which represents Markov random process, we call it "near-Markov approximation" after D. T. Gavel and D. M. Wiberg [56], who introduced the term. For this model the state transition matrix can be found as

$$
\hat{A}=\arg \min _{A}\left\langle\left\|\vec{\phi}_{t+1}-A \vec{\phi}_{t}\right\|_{2}^{2}\right\rangle,
$$

yielding

$$
\hat{A}=\left\langle\vec{\phi}_{t+1} \vec{\phi}_{t}^{T}\right\rangle\left\langle\vec{\phi}_{t} \vec{\phi}_{t}^{T}\right\rangle^{-1}
$$

where $\vec{\phi}_{t}$ are the coefficient vectors of the turbulence phase approximations (3.5). With the frozen flow hypothesis and bilinear spline approximation (3.9) assumed, the covariance matrices appearing in Eq. (5.2) take the form

$$
\begin{gathered}
\left\langle\vec{\phi}_{t+1} \vec{\phi}_{t}^{T}\right\rangle_{i j}=\left\langle\phi\left(x_{i}-v_{x} \Delta t, y_{i}-v_{y} \triangle t, t\right) \phi\left(x_{j}, y_{j}, t\right)\right\rangle, \\
\left\langle\vec{\phi}_{t} \vec{\phi}_{t}^{T}\right\rangle_{i j}=\left(C_{\phi \phi}\right)_{i j}=\left\langle\phi\left(x_{i}, y_{i}, t\right) \phi\left(x_{j}, y_{j}, t\right)\right\rangle,
\end{gathered}
$$

where $\phi(x, y, t)$ is a space-time phase distribution on each phase screen, $\left(x_{i}, y_{i}\right)$ are points of the spline grid, $\left(v_{x}, v_{y}\right)$ are the wind velocity components. These matrices can be evaluated theoretically in the case of piston- or point-removed Kolmogorov or von Karman turbulence by Eqs. (3.17) and (3.18), respectively. Note that the state transition matrix for several independent phase screens is block-diagonal with the blocks of the form given by Eq. (5.2).

With a known state transition matrix $\hat{A}$ the system driving noise steady state covariance matrix $C_{w w}$ can be found from the Lyapunov equation [66]

$$
C_{\phi \phi}=\hat{A} C_{\phi \phi} \hat{A}^{T}+C_{w w}
$$

The form of $C_{\phi \phi^{-}}$and $A$-matrices determined by Eqs. (5.2) - (5.4) guaranties that matrix $C_{w w}$ is positive semi-definite, which is necessary for the Kalman filter to be stable. 


\subsection{Kalman Filter equations.}

According to the Separation Principle, the KF reconstructor can be split into two stages: dynamic phase estimator to be derived from the orthogonality principle (3.58) and DM fitter given by Eq. (3.52), i.e. the same as that for the MV control. In the simulations below only estimation stage, i.e. the KF itself, will be considered, which will allow the lower limit for the AO system errors to be evaluated. The recursive solution to Eq. (3.58) is called the Kalman-Bucy equations [66]. With state vector $\vec{X}_{t}$ equal to the current turbulence vector $\vec{\phi}_{t}$ in accordance with the near-Markov model, these equations for the state estimate $\vec{X}_{t}$ and the estimator matrix $\hat{E}_{t}$ are

$$
\begin{gathered}
\hat{\vec{\phi}}_{t+1 \mid t}=\hat{A} \hat{\vec{\phi}}_{t \mid t}, \hat{\vec{\phi}}_{0 \mid 0}=\left\langle\vec{\phi}_{t}\right\rangle=0, \\
\hat{\vec{s}}_{t+1 \mid t}=G_{\phi} \hat{\vec{\phi}}_{t+1 \mid t}, \\
\hat{\vec{\phi}}_{t+1 \mid t+1}=\hat{\vec{\phi}}_{t+1 \mid t}+\hat{E}_{t}\left(\vec{s}_{t+1}-\hat{\vec{s}}_{t+1 \mid t}\right) ; \\
C_{t+1 \mid t}^{\epsilon}=\hat{A} C_{t \mid t}^{\epsilon} \hat{A}^{T}+C_{w w}, C_{0 \mid 0}^{\epsilon}=C_{w w}, \\
\hat{E}_{t+1}=C_{t+1 \mid t}^{\epsilon} G_{\phi}^{T}\left(G_{\phi} C_{t+1 \mid t}^{\epsilon} G_{\phi}^{T}+C_{v v}\right)^{-1}, \\
C_{t+1 \mid t+1}^{\epsilon}=C_{t+1 \mid t}^{\epsilon}-\hat{E}_{t+1} G_{\phi} C_{t+1 \mid t}^{\epsilon},
\end{gathered}
$$

where $C^{\epsilon}$ is the estimation error covariance matrix, subscripts $t+1 \mid t$ and $t+1 \mid t+1$ stand for the estimate at time $t+1$ given the data up to time $t$ or $t+1$, respectively. Note that the steady-state solution $\hat{E}_{\infty}$ of Eq. (5.10) can be taken for the time-invariant Kalman estimator.

An important fact from the linear estimation theory is that for the system driven by the dynamics model (3.56) the optimal linear state estimate $\hat{\phi}_{t+n \mid t}$ (n-step prediction) is given by [64]

$$
\hat{\vec{\phi}}_{t+n \mid t}=\hat{A}^{n} \hat{\vec{\phi}}_{t \mid t} .
$$


It should be noted that Eq. (5.12) is valid independently of the way the current state estimate is obtained, i.e. it is good for both KF and MV approaches. According to Eq. (5.12), the n-step rms prediction error of the application the near-Markov state transition matrix $\hat{A}$ obtained in the previous section is

$$
\epsilon=\left\langle\left\|\vec{\phi}_{t+n}-\hat{A}^{n} \vec{\phi}_{t}\right\|^{2}\right\rangle^{1 / 2}
$$

where $\vec{\phi}_{t}$ is a vector of bilinear phase approximation coefficients, that is, the turbulence phase values at the discrete grid points at time $t, \vec{\phi}_{t+n}$ is a vector of phase values of wind-shifted turbulence at the same grid points at time $t+n$. Fig. 5.1 shows the graphs of prediction error (5.13) as a function of a delayed time samples number $n$ for a single moving discrete Kolmogorov phase screen consisting of 248 grid points imbedded into 5.1-meter circular pupil of Palomar telescope. The phase error due to pure lag is shown on the same figure for comparison. As it is seen from the figure, the prediction ability of the near-Markov model is very moderate for small, one- or two-sample delays typical for AO control, and becomes relatively better for the bigger delays. Nevertheless, as it will be shown in the next section, AO systems simulations show adequate ability of the near-Markov model to compensate for the 2-sample latency in both SCAO and MCAO systems.

\subsection{Simulation results.}

The performance of the MV and KF estimators was simulated for two AO systems.

1. Multi conjugate Gemini South telescope with primary mirror diameter $8 \mathrm{~m}$ and secondary mirror diameter $1.22 \mathrm{~m}$. The AO system has one $90 \mathrm{~km}$ sodium laser quide star (LGS) WFS on axis, three LGS WFSs at 30 arcsec off axis arranged in a equilateral triangle (subaperture size $0.5 \mathrm{~m}$ ), and three natural guide star (NGS) WFS for tip-tilt correction at 30 arcsec off axis arranged in equilateral triangle. FoV 


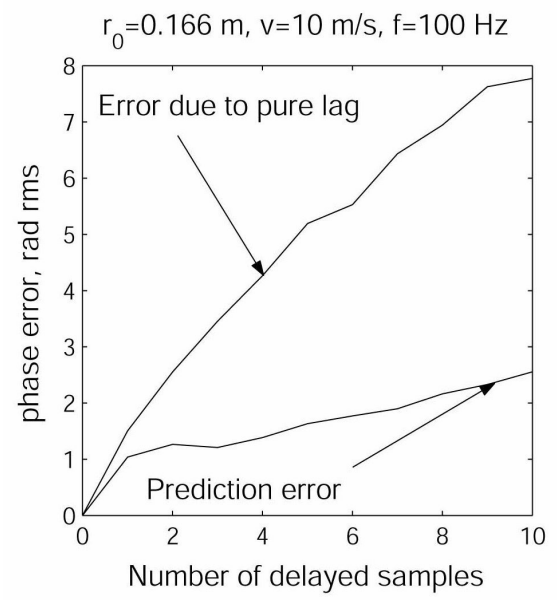

Figure 5.1: Turbulence phase prediction error as a function of delayed samples number for the near-Markov model computed by Eq. (5.13). Piston-removed Kolmogorov turbulence with Fried parameter $r_{0}=0.166 \mathrm{~m}$ is assumed. Wind speed is equal to $|\vec{v}|=10 \mathrm{~m} / \mathrm{s}$, the system sampling rate is $f=100 \mathrm{~Hz}$. A single discrete phase screen with 248 equidistant phase grid points fill the 5.1-meter circular pupil of the Palomar telescope. 
consisted of nine scientific directions arranged equidistantly in $1^{\prime} \times 1^{\prime}$, as shown on Fig. 5.2. 7-layer Kolmogorov turbulence model (Table 5.1) for average Cerro Pachon site seeing conditions [59] with integral Fried parameter $r_{0}=0.166 \mathrm{~m}$ and Greenwood frequency $f_{G}=29 \mathrm{~Hz}(\lambda=0.55 \mu \mathrm{m})$ was assumed.

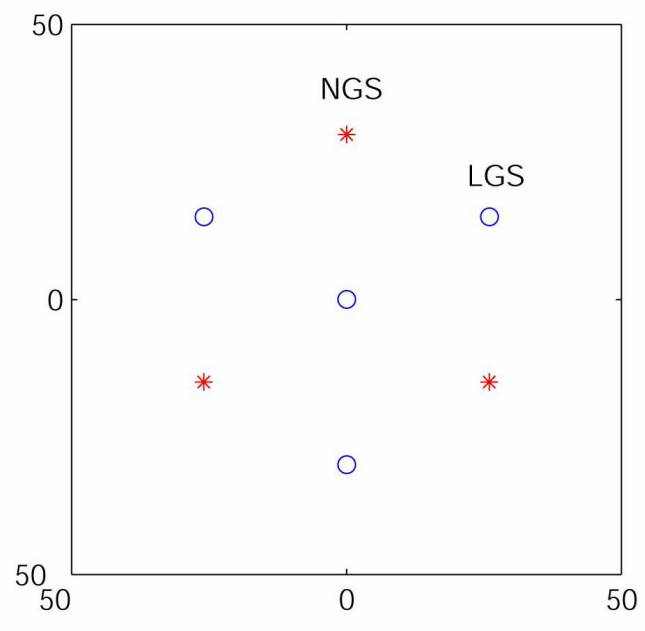

Angular position, arc min

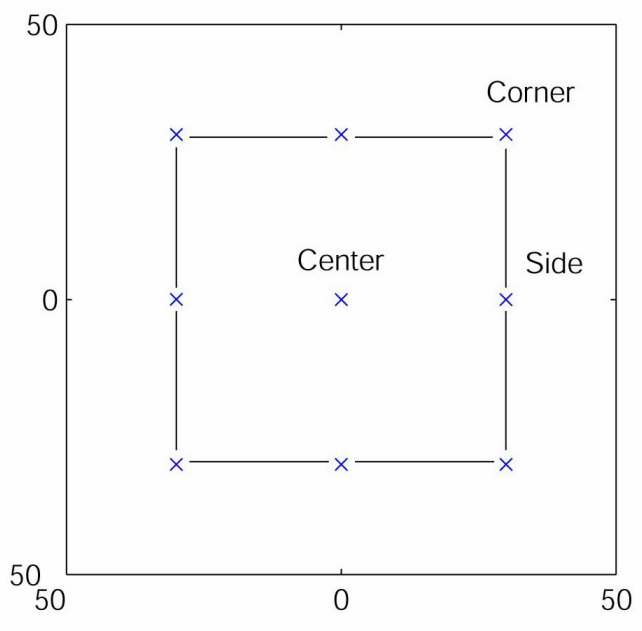

Angular position, arc min

Figure 5.2: Guide stars (left panel) and field of view (right panel) alignment for Gemini South MCAO system.

Turbulence phase on all phase screens was estimated on the equidistant square grids with the grid size $0.5 \mathrm{~m}$. Typical phase screen grid with the beamprint and the LGS WFS subaperture geometries are shown on Fig. 5.3.

2. Single conjugate Palomar telescope AO system with primary mirror diameter 5.1 $\mathrm{m}$ and secondary mirror diameter $0.77 \mathrm{~m}$. One $90 \mathrm{~km}$ sodium LGS WFS (subaperture size $0.32 \mathrm{~m}$ ) and one single-subaperture NGS WFS, both on optical axis. Field of view consisted of one scientific direction along optical axis. Turbulence was modelled as a single ground-based screen approximation of the same Cerro Pachon 7-layer profile with integral Fried parameter $r_{0}=0.166 \mathrm{~m}$, equivalent wind speed $V=10 \mathrm{~m} / \mathrm{s}$ and 


\begin{tabular}{|c|c|c|}
\hline Layer altitude, $\mathrm{km}$ & Relative weight & Wind velocity, m/s \\
\hline 0.0 & .646 & 6.6 \\
1.8 & .080 & 12.4 \\
3.3 & .119 & 8.0 \\
5.8 & .035 & 33.7 \\
7.4 & .025 & 23.2 \\
13.1 & .080 & 22.2 \\
15.7 & .015 & 8.0 \\
\hline
\end{tabular}

Table 5.1: Cerro Pachon 7-layer turbulence model.

Greenwood frequency $29 \mathrm{~Hz}(\lambda=0.55 \mu \mathrm{m})$. The turbulence phase was estimated on an equidistant square grid with the grid size $0.32 \mathrm{~m}$.

Open-loop operation was assumed. Although the KF algorithm described by Eqs. (5.6) - (5.11) can work in closed-loop mode with only minor modifications, the questions of closed-loop stability and sensitivity to system errors were not considered. The MV controller used in the simulations is described by Eqs. (3.51), (3.52). Full matrix approach was used for MV control, i.e. no sparse methods and approximations were employed.

Monte-Carlo simulations have been performed for the controllers with two-frame delay (one frame for $\mathrm{CCD}$ readout and one for computation) operating at 500, 250 and $125 \mathrm{~Hz}$ sampling rates. WFS noise was set to $1 \mathrm{rad} \mathrm{rms}$ per subaperture $(\lambda=0.5$ $\mu \mathrm{m})$ for $500 \mathrm{~Hz}$ and was reduced accordingly to 0.71 and $0.5 \mathrm{rad}$ per subaperture for lower sampling rates to model working with the guide stars of fixed magnitude. The integrated aperture phase residual

$$
\left\langle\left\|\vec{\epsilon}_{t}\right\|_{\tilde{W}}^{2}\right\rangle_{t}=\left\langle\left\|H_{\phi} \vec{\phi}_{t}-H_{\phi} \hat{\vec{\phi}}_{t}\right\|_{\tilde{W}}^{2}\right\rangle_{t}
$$

for different FoV directions averaged over simulation time (transient excluded) has been 

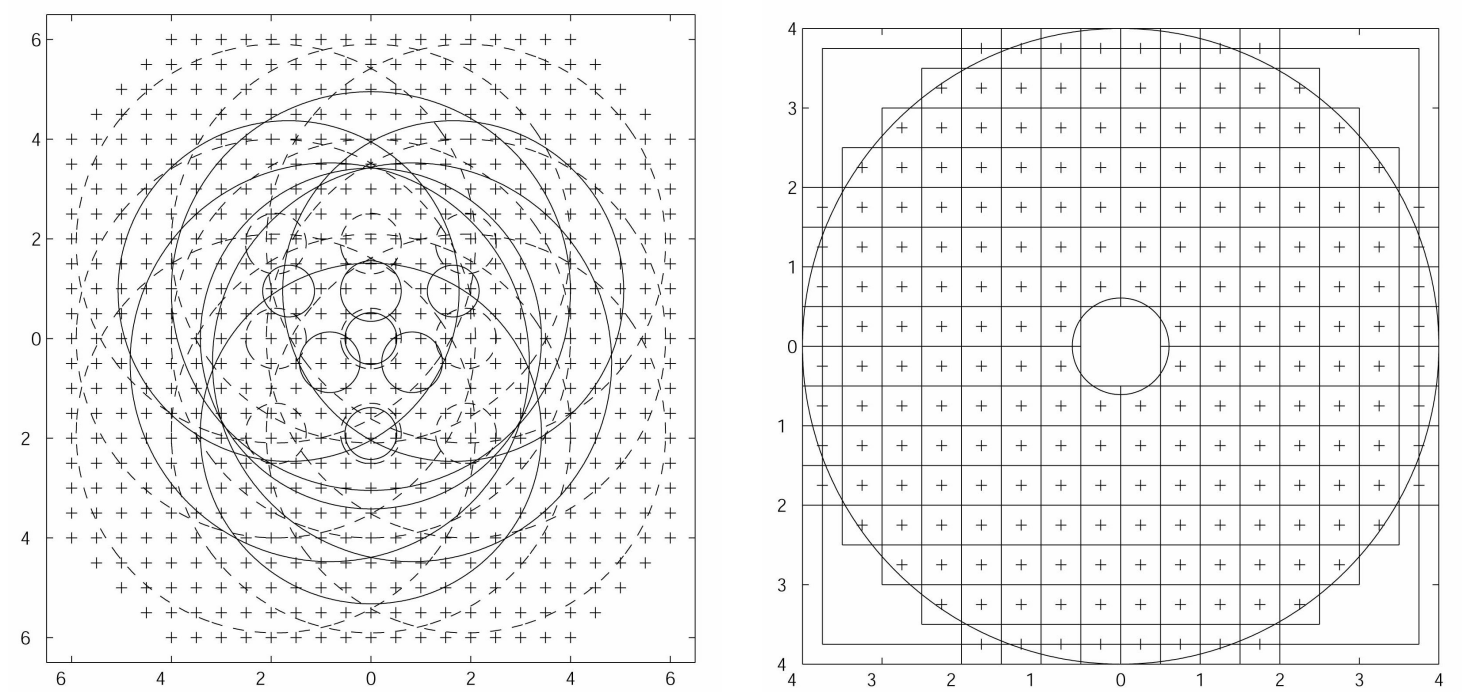

Figure 5.3: Left panel: fifth phase screen reconstruction phase grid and beamprint. The circles are projections of the annular aperture onto the screen along both scientific and guide stars directions. Right panel: LGS WFS subaperture geometry for Gemini South MCAO. Big and small circles are the outer and inner borders of the annular main aperture of the system.

taken as a measure of estimators performance. Residual vector $\vec{\epsilon}_{t}$ was estimated on the square aperture grid with grid sizes $0.04 \mathrm{~m}$ (Palomar), $0.0625 \mathrm{~m}$ (Gemini).

The input random phase screens were generated using Fourier transform method with tilt correction [41]. Wind shift was performed in frequency domain by the means of the Fourier shift theorem (3.59). Input phase grid size was taken equal to one eighth of the DM actuator grid size to account for higher spatial frequencies present in real turbulence (see Sec. 3.7). To ensure the best fit of the statistical model employed in $\mathrm{KF}$ and $\mathrm{MV}$ estimators to the input, the covariance matrices appearing in Eqs. (3.51), (5.9), (5.2) were computed directly from sample statistics of the phase screen generator. Typical simulation results for Palomar and Gemini South AO systems are 
shown on Figure 5.4. The averaged estimation errors for different estimation strategies and different $\mathrm{AO}$ systems are collected in Tables 5.2 and 5.3.
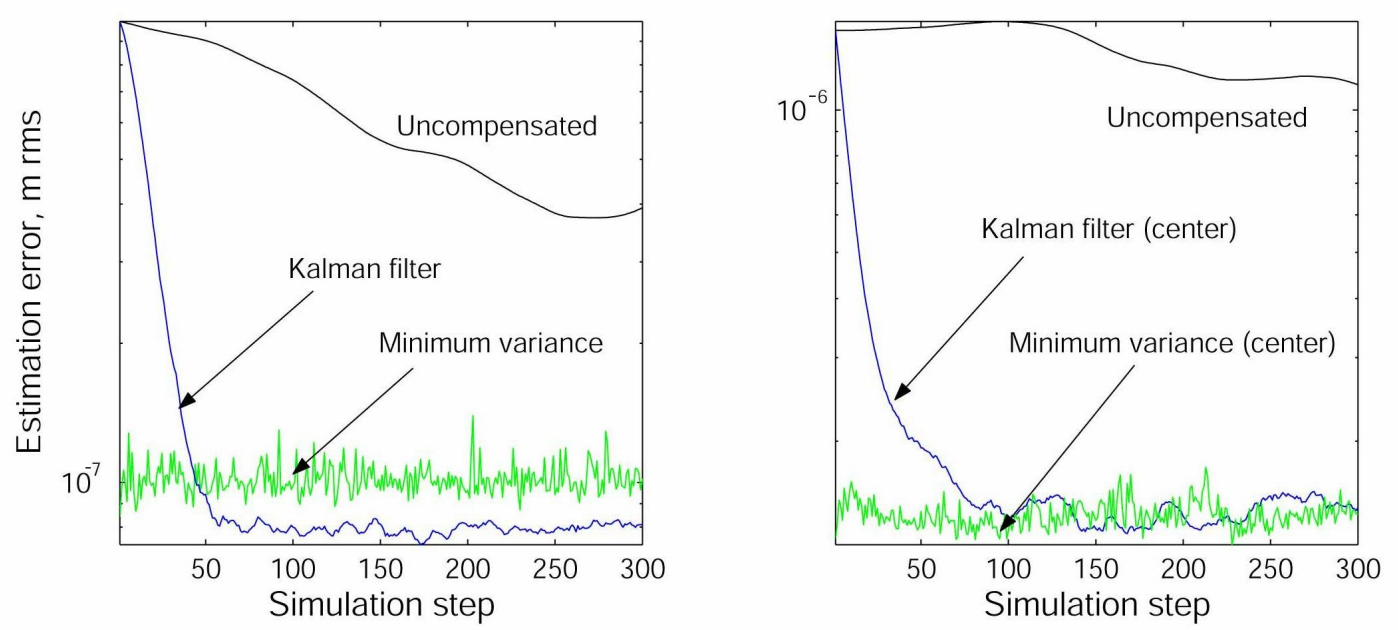

Figure 5.4: Estimation error time evolution graphs obtained by Monte-Carlo simulations of the Palomar (left panel) and Gemini South (right panel) AO systems. Sampling rate $500 \mathrm{~Hz}$. The KF curves correspond to the case of 2-step prediction, whereas MV curves correspond to pure 2-step lag.

Results for the SCAO show superior performance of KF in all cases, which is expected and in agreement with the results of the previous work $[56,57]$. The lag compensation effect of even the moderately accurate near-Markov predictor is apparent. Note the only slight performance deterioration of KF with predictor for reduced sampling rates. This clearly shows how the lag compensation allows to benefit from WFS noise reduction due to increased WFS integration time.

Results for MCAO, however, do not show an advantage for the KF approach. In all cases KF performance is only slightly better than that of MV with pure 2-step lag and even worse than that of MV with 2-step temporal prediction. The worse KF algorithm's correction for off-axis points is also apparent. This fact can be accounted for higher 


\begin{tabular}{|c|c|c|c|}
\hline & $500 \mathrm{~Hz}$ & $250 \mathrm{~Hz}$ & $125 \mathrm{~Hz}$ \\
\hline KF, prediction on & 79 & 82 & 88 \\
\hline KF, prediction off & 84 & 103 & 157 \\
\hline MV, prediction on & 98 & 90 & 91 \\
\hline MV, prediction off & 101 & 108 & 157 \\
\hline
\end{tabular}

Table 5.2: Estimation errors (in nanometers rms) for Palomar AO system averaged over simulation time for KF and MV with 2-step prediction switched on/off.

\begin{tabular}{|c|c|c|c|}
\hline & $500 \mathrm{~Hz}$ & $250 \mathrm{~Hz}$ & $125 \mathrm{~Hz}$ \\
\hline KF, prediction on & $141 / 182 / 292$ & $141 / 180 / 326$ & $143 / 184 / 320$ \\
\hline KF, prediction off & $143 / 185 / 295$ & $153 / 193 / 333$ & $199 / 232 / 349$ \\
\hline MV, prediction on & $134 / 169 / 248$ & $132 / 170 / 277$ & $141 / 186 / 315$ \\
\hline MV, prediction off & $141 / 172 / 249$ & $146 / 181 / 282$ & $193 / 228 / 340$ \\
\hline
\end{tabular}

Table 5.3: Estimation errors (in nanometers rms, center/side/corner of FoV) for Gemini South AO system averaged over simulation time for KF and MV with 2-step prediction switched on/off.

sensitivity of MCAO KF algorithm to the assumed statistical model discrepancies (upsampling in our case). But the most surprising result is that the performance of MV algorithm with state prediction added can be comparable or even better than that of KF. This makes questionable the use of the more computationally expensive KF algorithm and, on the other hand, calls for effective turbulence predictors. 


\subsection{Conclusions.}

The ability of zonal KF with Near-Markov temporal dynamics model to effectively estimate atmospheric turbulence has been demonstrated for both SCAO and MCAO cases though $\mathrm{KF}$ performance for MCAO proves to be worse than expected. It has also been shown that classical MV estimator performance can be significantly improved by adding a temporal predictor to it. It is common knowledge that the success of Kalman filter implementation largely depends on the choice of state dynamics model, which affects both estimation and prediction performance. Although the feedback nature of KF can compensate to some extent for the model discrepancies, simple AR models may not be capable to represent the real random process estimated thus reducing the effectiveness of KF approach. This can be the case for these simulations because the moderate accuracy of the Near-Markov dynamics model may well be inadequate in $\mathrm{MCAO}$ case. Another possible reason for worse KF performance in multiple conjugate case is relatively higher sensitivity of KF to the errors introduced by the high frequency part of the input. Finding ways for KF MCAO performance improvement is the goal for further research. 


\section{CHAPTER 6}

\section{Sparse Predictive Minimum Variance \\ Controller.}

As it has been shown in the previous chapter, the non-dynamic Minimum Variance controller with a prediction algorithm added to it performs surprisingly well even in comparison to the dynamic Kalman Filter. This gives additional motivation for working on predictive MV control algorithms for MCAO. The serious drawback of the near-Markov turbulence dynamics model used for temporal prediction in the KF-based approach is that the state transition matrix $\hat{A}$ is fully populated, which brakes the $\mathscr{O}(N)$ behavior of sparse MV algorithm described in detail in Sec. 4.1. In this section we will show that it is possible to use a temporal prediction strategy, which is not only $\mathscr{O}(N)$ but also requires no additional computations to perform phase prediction.

\subsection{Idea of the sparse phase prediction.}

According to Sec. 5.2, the phase estimate $\vec{\phi}_{t}$ at time $t$ based on the concatenation vector $\vec{S}_{t-k}$ of the measurements up to time $t-k$ consists of two steps. The first step is to find the phase estimate at time $t-k$ :

$$
\hat{\vec{\phi}}_{t-k}=\mathscr{E} \vec{S}_{t-k}
$$


where $\mathscr{E}$ is a general phase estimation operator. The second step is the $k$-step prediction:

$$
\hat{\vec{\phi}}_{t}=\mathscr{A}^{k} \hat{\vec{\phi}}_{t-k}
$$

where $\mathscr{A}$ is a general one-step prediction operator, which in the case of the frozen flow hypothesis assumed is nothing but the pure shift operator, generally nonlinear. When making phase estimation, as it was done in the previous chapter, some linear approximation to the $\mathscr{A}$, such as the near-Markov (see Sec. 5.1), is necessary to come up with a linear controller. The situation is different if the full control is considered, when the DM fitting

$$
\hat{\vec{a}}_{t}=\hat{F} \hat{\vec{\phi}}_{t}
$$

follows the phase estimate. Eq. (3.52) for the fitting matrix $\hat{F}$ contains the phase-tophase interaction matrix $H_{\phi}$ (see Eq. (3.40)) that projects vector $\hat{\vec{\phi}}_{t}$ of phase estimate values assigned to the points of the phase screen grids, like those shown on Fig. 4.7, onto the main aperture grid. If the phase estimate vector $\hat{\vec{\phi}}_{t-k}$ is available, then, according to the frozen flow hypothesis, the best possible $k$-step prediction of it is the same vector $\hat{\vec{\phi}}_{t-k}$ but assigned to the phase screen grids shifted from their initial positions by

$$
\triangle \vec{x}=k \triangle t \vec{v}
$$

where $\vec{v}$ is the wind speed, $\Delta t$ is the sampling interval. This simply results in a new matrix $H_{\phi}^{\text {pred }}$, which projects onto the main aperture the phase estimate assigned to the shifted phase grids. Correspondingly, a new fitting matrix $\hat{F}_{\text {pred }}$, which fits the DM commands to the predicted phase estimate, can be computed, with $H_{\phi}$ replaced by $H_{\phi}^{\text {pred }}$ in Eq. (3.52). A few comments need to be made:

- The initial phase grids should be made slightly larger than the aperture beamprint on the screen to fill the gap created by the finite point grid on the "downwind" side of the beamprint after the grid shift. Fig. 6.1 shows the example of the extra 
points addition to the ground layer phase grid that make a "guard band" outside the aperture. Since the guard band points are outside the aperture beamprint,

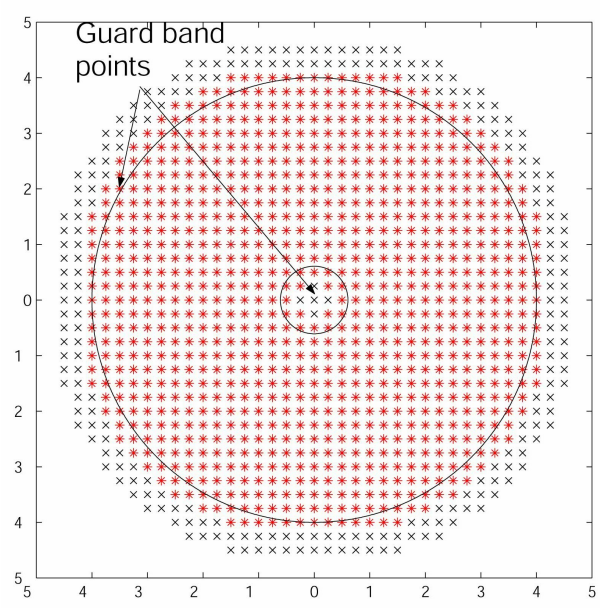

(a)

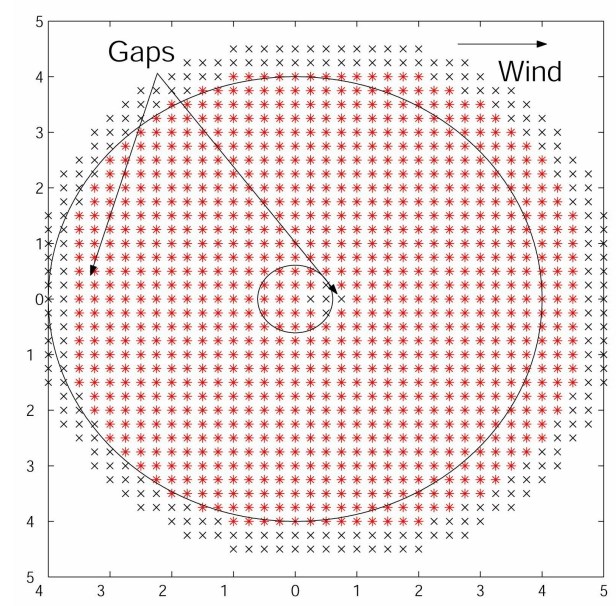

(b)

Figure 6.1: Illustration of the guard band addition to the phase grid. (a): initial position of the grid. Main grid points are denoted by “*”. Guard band points are denoted by "x". (b): grid position after a wind shift.

they are not coupled to the WFS measurements and phase values at these points are only the statistical extrapolation of the phase within the beamprint by means of the regularization term $C_{\phi \phi}^{-1}$ in Eq. (3.51) or its sparse approximation $\eta L^{T} L$ (4.4). This results in an error in the otherwise exact phase prediction, which must be small for the small wind shifts, when the gap filled with guard band points is small relatively to the rest of the aperture. This condition is well satisfied for the big apertures of giant telescopes. Note that, in the absence of the regularization term, the phase values on the guard band would be just zeros, thus significantly increasing the estimation error.

- Another prediction error source is the frozen flow assumption, which does not 
exactly hold for real turbulence over any but the shortest time intervals.

- Since computation of the matrix $H_{\phi}^{\text {pred }}$ needs just specification of the new grid, its computational cost is the same as that for the no prediction case save for a small overhead due to guard band addition. Therefore, the entire sparse predictive MV controller still has computational cost around $\mathscr{O}(N)$.

\subsection{Simulation results.}

The same two Palomar SCAO and Gemini South MCAO systems as those in the previous chapter were simulated to evaluate the performance of the Predictive Pseudo Open Loop Controller (P-POLC). The $z$-domain diagram of it is shown on Fig. 6.2.

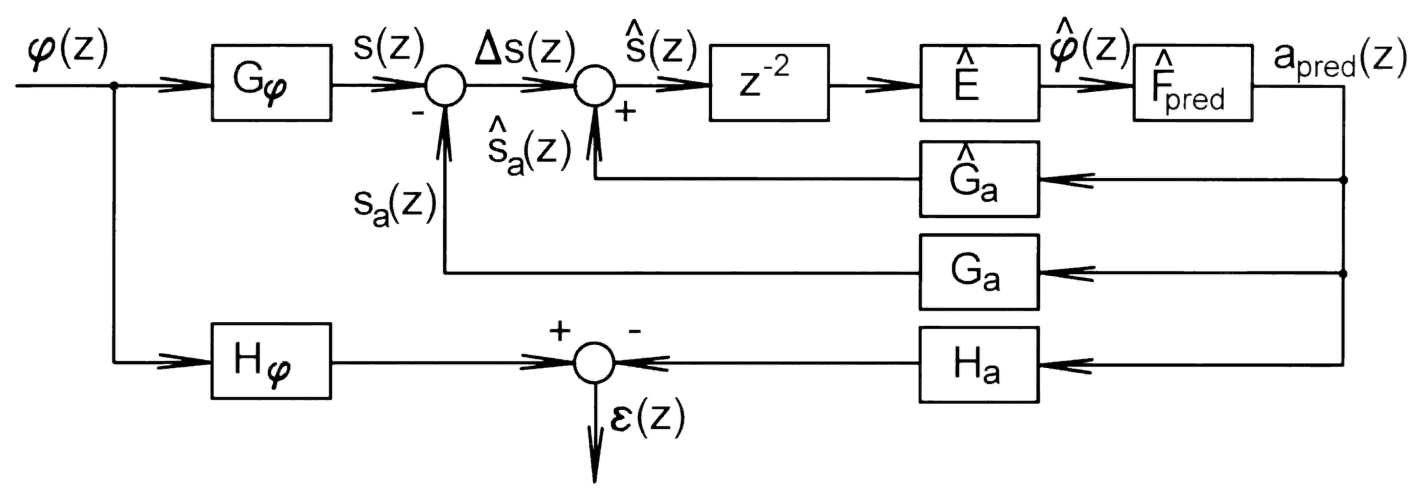

Figure 6.2: $z$-domain block diagram of the MV Pseudo Open Loop Controller with the sparse predictor.

In contrast to the analogous system from Chapter 4, a perfectly aligned system was considered, which is reflected by the absence of the error inputs in the diagram. Another difference is that more realistic simple 2-frame delay in the measurement acquisition is considered instead of the negative feedback integrator (4.34) used to model the delay in the POLC system of Chapter 4. The phase prediction is implemented by 
the replacement of fitting matrix $\hat{F}$ by the $\hat{F}_{\text {pred }}$ computed as explained above. Much lower sampling rate of $100 \mathrm{~Hz}$ was used, in contrast to the $800 \mathrm{~Hz}$ sampling rate in the case of POLC of Chapter 4. This accounts for a different and more effective paradigm of predictive control. Namely, in conventional adaptive optics an integrator-type lead compensator is used with bandwidth equal to $\sim 3 f_{G}$ (see Sec. 4.4) and sampling rate equal to $\sim 30 f_{G}$ to avoid possible instability due to the lag. No information about turbulence dynamics is employed. In the approach of this chapter, the $3 f_{g} \approx 100$ $\mathrm{Hz}$ sampling rate is used together with effective phase predictor based on the known turbulence dynamics used for lag compensation. The advantage of this approach is obvious: the dramatic sampling rate reduction leads to the corresponding reduction of computational complexity. In addition, the increased integration time leads to bigger signal-to-noise ratio in the WFS channel.

To accurately simulate the turbulence dynamics the Fourier transform based input phase screens generation with tip-tilt correction [41] and direct phase grid shifting (see Sec. 3.7) was used. The spatial sampling on the generated phase screens was equal to one eighth of that on the reconstruction phase screens. Direction and magnitude of the wind shift were taken the same for all screens and equal to one simulation grid spacing, which is equivalent to the wind speed $6.25 \mathrm{~m} / \mathrm{s}$ for the sampling rate of $100 \mathrm{~Hz}$.

The typical results of P-POLC reconstructor Monte-Carlo simulations are presented on Fig. 6.3, where both Palomar SCAO and Gemini South systems reconstruction phase error time evolutions computed via Eq. (3.42) are shown. The system performance is shown for the cases of temporal prediction turned on/off.

Conclusions that can be drawn from the simulations can be summarized as follows:

- The AO systems are stable for the prediction turned both on and off. The stability in the second case must be accounted for the robustness of POLC shown in Chapter 4. 

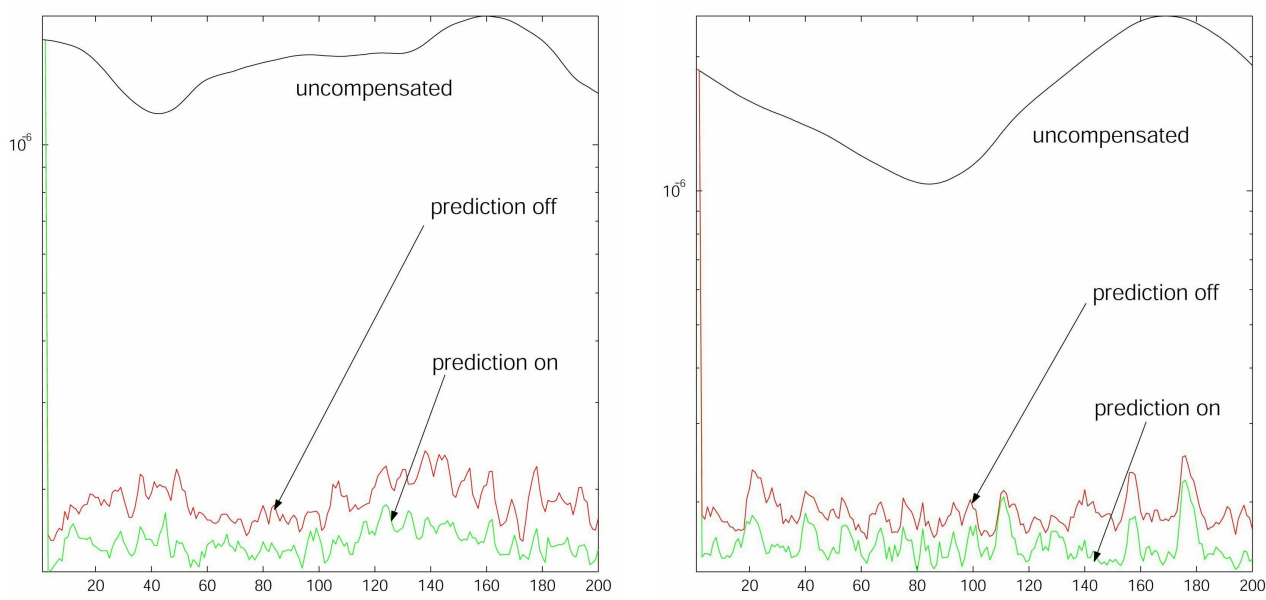

Figure 6.3: Reconstruction error time evolution graphs obtained by Monte-Carlo simulations of the Palomar (left panel) and Gemini South (right panel, center of FoV) AO systems driven by sparse MV controller with 2-step prediction turned on/off.

- The reconstruction errors for Palomar and Gemini South AO systems averaged over simulation time with 2-step prediction switched on/off are given in the Table 6.1. The positive influence of the prediction is obvious and the results are in agreement with the ones obtained in the previous chapter for the MV reconstructor with near-Markov prediction model. The difference is, however, in the significantly lower computational cost of the sparse predictive algorithm.

\begin{tabular}{lcc}
\hline \hline & $\begin{array}{c}\text { Palomar } \\
\text { center of FoV }\end{array}$ & center/side/corner of FoV \\
\hline prediction off & 157 & $189 / 212 / 256$ \\
prediction on & 102 & $160 / 186 / 234$ \\
\hline \hline
\end{tabular}

Table 6.1: Reconstruction errors (in nanometers rms) for Palomar and Gemini South AO systems averaged over simulation time with 2-step prediction switched on/off. 


\section{CHAPTER 7}

\section{Conclusions and the future work.}

In this dissertation, the following new results on the design and performance modeling of effective control algorithms for the large scale AO systems for the new generation of giant astronomical telescopes has been obtained.

1. Robustness of the Minimum Variance Pseudo Open Loop Control (POLC) algorithm proposed by B. Ellerbroek and C. Vogel [47] as a simple method for the stable MCAO system operation in closed loop has been carefully investigated. The initial assumption about the precise knowledge of the system for the POLC to work has been disapproved and high robustness of this algorithm against system errors has been demonstrated. Currently, a sparse $\mathscr{O}(N)$ version of POLC described in Sec. 4.1 is considered the main control concept for the AO in the Thirty Meter Telescope project [1].

2. The limiting performance of the non-dynamic Minimum Variance and dynamic Kalman Filter (KF) based phase estimation algorithms for MCAO has been evaluated in the framework of zonal control. The validity of near-Markov autoregressive phase dynamics model has been tested. It has been found that despite the larger amount of information involved in dynamic KF control there is no performance improvement in comparison to the much simpler Minimum Variance algorithm in the case of MCAO. This is most likely due to the lack of an 
adequate linear model for the turbulence dynamics. However, more careful investigation of the $\mathrm{KF}$ algorithm is needed. The positive outcome of this work is the surprisingly good ability of the simple near-Markov turbulence dynamics model to compensate for the 2-frame latency in the system, which opens the way to further complexity reduction of the AO control.

3. The low complexity sparse predictive MV control algorithm has been proposed. It has been shown that turbulence phase temporal prediction can be done very effectively and is able to significantly reduce the error due to the measurement and control latency always present in real AO systems. It has been shown by Monte-Carlo simulations that with the phase prediction added it is possible to significantly reduce the sampling rate in comparison with usual servo compensator approach without loss of stability and accuracy of wave front reconstruction. It should be pointed out that the corresponding complexity reduction due to smaller sampling rate can be achieved without any additional computational burden per single sample.

4. The last result that could be mentioned is the development of the MCAO system simulation software capable to evaluate the performance and robustness against numerous errors of the AO systems driven by Least Squares, Minimum Variance and Kalman Filter controllers.

The results presented are only a little fraction of the research that can be done in the vast area of the Adaptive Optics control. The following ramifications of the research work in this area can be, in the author's opinion, pursued in the future.

1. It is a well known fact that the Taylor Frozen Flow Hypothesis described in Sec. 2.3 is not valid for the large apertures. During the time necessary for wind to blow the turbulence across more than 10-meter aperture the "boiling" fraction of 
turbulence temporal dynamics becomes appreciable, so the Frozen Flow Hypothesis becomes an inaccurate assumption for the new giant telescopes with primary mirror diameters in excess of $10 \mathrm{~m}$. All the simulations presented in this dissertation are made with the Frozen Flow assumption for the AO systems with the main apertures no more than 8 meters, and scaling these results to the bigger diameters can be incorrect. On the other hand, methods of phase screen generation that account for the "boiling fraction" exist $[17,39]$. Their software implementation, verification and routine use in the large-scale AO systems simulations is the important task for the future.

2. Another unsolved problem is the optimal discrete representation of the continuous phase distributions through Eq. (3.5). It is well known that neither Zernike polynomials nor bilinear splines are optimal basis sets to approximate the random phase functions with Kolmogorov or von Karman statistics in the sense that for a fixed number of terms in Eq. (3.5) the approximation error is minimized. Other basis sets can be considered for the purpose. The multi-grid expansions and control algorithms $[10,11]$ based on them appear to be one of the most interesting directions of the future research in $\mathrm{AO}$ control. This makes it possible the design of new low-complexity control algorithms finely tuned [8] to achieve optimal performance on different spatial frequency components of the signal. The wavelet-based expansions are the natural choice for the multi-grid algorithms but, strangely enough, to the author's best knowledge, wavelets has never been considered as the basis sets for AO.

3. It is interesting to notice that the sparse approximation (4.4) of the inverse turbulence phase covariance matrix $C_{\phi \phi}^{-1}$ formally turns the minimum variance phase estimation problem (3.53) into classical LS problem with Laplacian-square Tikhonov regularization operator like that described by Eq. (3.48). The use 
of a priori information about the turbulence statistics gets formally reduced to just choosing the appropriate regularization operator, which does not depend on the turbulence strength, and regularization coefficient $\eta$, which depends on the turbulence only very weakly. This similarity of the MV approach and the much simpler least squares technique has been pointed out recently by C. Vogel and Q. Yang [45]. They proposed not to separate the control problem into estimation and fitting stages but to solve the following regularized least squares problem

$$
\hat{a}_{t}=\arg \min _{\vec{a}}\left(\vec{s}_{t}-G_{a} \vec{a}+\alpha I+\eta L^{T} L\right),
$$

where $L$ is the discrete Laplacian matrix and $\alpha, \eta$ are two regularization parameters, whose optimal values can be easily found by trial and error. The information about turbulence statistics is used here only implicitly in the form of "right" regularization operator. Note that Eq. (7.1) results in the same sparse reconstruction algorithm that can be effectively implemented with the aid of multi-grid iterative methods. In the case of SCAO this approach is reported to be as accurate as the full MV reconstructor. It would be interesting to extend the idea to the MCAO. If the same result holds for MCAO, since the size of DM command vector $\vec{a}_{t}$ is at most one half the size of turbulence phase vector $\vec{\phi}_{t}$ estimated in the MV control and because the fitting stage can be excluded, one can expect the additional fourfold complexity reduction. The difficulty is, however, expected with the prediction implementation. In the case of LS control DM commands vector $\vec{a}_{t}$ has to be predicted. But, its temporal behavior cannot be predicted by the simple shift operator as in the case of the phase estimate for the absence of one-to-one correspondence between turbulence and DM commands for MCAO. Some approximate linear, desirably sparse, model for DM commands temporal dynamics needs to be developed instead. This represents another important ramification of the future research. 
4. As it has been shown in Chapter 5 the accurate linear models for turbulence dynamics or the dynamics of turbulence-driven quantities like DM command vector become crucial for further progress of the control algorithms for AO. These models can be derived either theoretically or, which is better, directly from the data sequences measured by the AO system wave front sensors by statistical system identification methods as it has been described, for instance, in [12]. The second way is preferable because no assumptions, like layered atmosphere or Frozen Flow Hypothesis, have to be made about the physics of turbulence. On the other hand, the main drawback of the work [12] is that the ARMA model for the turbulence dynamics proposed there is not sparse. The existence of simple but adequate linear models for turbulence temporal dynamics depends entirely on the physical nature of turbulence and is, for now, an open question.

5. It was assumed for both MV and KF control approaches considered in this dissertation that the turbulence characteristics, such as $C_{n}^{2}$ and wind velocities profiles, are known in advance from the independent measurements and do not change during the exposure time. This assumption is quite restrictive in practice but can be eliminated by the use of the well known adaptive control approach, when the sample statistics of the measurements are continuously accumulated within a moving time window and used for the control commands estimation. Theory and mathematical tools of the adaptive control are a very well developed part of modern control theory. A simple version of adaptive control for SCAO in the framework of the modal approach with decoupled channels has been investigated in a series of papers by J. S. Gibson et. al. [7]. Implementation of the adaptive control in the zonal framework by combining the sparse methods described in this dissertation with the existing mathematical methods for the systems with multiple coupled channels is another promising branch of the future research. 
6. One more important observation about AO control is its fuzzy nature. Indeed, because of the statistical nature of the input signal and a number of the technical errors the DM correction cannot be applied in exact way. Simple calculation shows that if the local wave front residual error does not exceed $\lambda / 10 \mathrm{rms}$, which corresponds to very good performance, and DM dynamic range is a few wavelengths, the number of distinct positions of each actuator is no more than 100 . The DM commands calculated on a standard computer using 8-byte arithmetics with billions of distinct positions allowed seem to be a great waste of resources. The new methods are necessary to take the full advantage of the fuzzy nature of the AO control, which can lead to a huge leap in the technology with respect to complexity reduction and simplicity of hardware implementation. It should be pointed out that simple reduction of the word length used in the computations by conventional algorithms does not solve the problem for it may lead to numerical instability because of the ill-conditioned equations involved. The use of statistical methods for creation the sets of fuzzy decision rules that can replace the deterministic algorithms of AO control is another nontrivial research task. 


\section{Appendix A. \\ MUlTi-GRID RECURSIVE LINEAR SOLVERS.}

As it was pointed out in Sec. 4.1.2, iterative methods have poor convergence rate if applied to ill-conditioned equation systems. On the other hand, it was observed that the way the standard iterative linear solvers like Jacobi, Gauss-Seidel or conjugate gradients [67] act on the ill-conditioned matrix can be characterized as "stagnation". The high-frequency components of the solution error are effectively dumped after a few iterations, whereas the low-frequency error components are very slow to converge. For this behavior the iterative techniques are called "smoothers". The simple idea of the multi-grid (MG) methods [69] is to project the solution error on successively coarser grids and apply the smoother iterations to damp the error components that are of relatively low-frequency on a fine grid but become of relatively high frequency on a coarse grid. The second key idea of MG methods is to solve directly, i.e. non-iteratively, the equation system on the coarsest grid. This solver will null the lowest frequency error component remained after smoothers application and will not increase the overall computational complexity because the coarsest grid equation system has small size. Thus, multi-grid is a very effective combination of iterative and non-iterative methods.

The MG method for solving a linear system of equations $A \vec{x}=\vec{b}$ defined on a computational grid $\Omega$ requires:

1. A nested sequence of increasingly coarse grids $\Omega=\Omega_{1}, \ldots, \Omega_{L}$. On each grid, the set of restricted equation matrices $A=A_{1}, \ldots, A_{L}$, algebraic residuals $r_{l}$ and solution errors $e_{l}$, such that $A_{l} e_{l}=r_{l}, l=1, \ldots, L$, is defined.

2. The down- and up-sampling operators $I_{l}^{\downarrow}, I_{l}^{\uparrow}$ that project the matrices and vectors 
onto coarser and finer grids

$$
\begin{aligned}
& \vec{v}_{l+1}=I_{l}^{\downarrow} \vec{v}_{l}, A_{l+1}=\left(I_{l}^{\downarrow}\right)^{T} A_{l} I_{l}^{\downarrow}, \\
& \vec{v}_{l-1}=I_{l}^{\uparrow} \vec{v}_{l}, A_{l-1}=\left(I_{l}^{\uparrow}\right)^{T} A_{l} I_{l}^{\uparrow} .
\end{aligned}
$$

These operators are usually taken such that $I_{l}^{\uparrow}=\left(I_{l}^{\downarrow}\right)^{T}$ in order to preserve the symmetry of matrices $A_{l}$.

3. Smoother, i.e. iterative method to solve systems $A_{l} \vec{e}_{l}=\vec{r}_{l}$ that rapidly eliminates high frequency components from the solution error $\vec{e}_{l}$. Since in the case of MV wave front reconstructor for MCAO the system matrices are symmetric the preconditioned conjugate gradient $(\mathrm{CG})$ method is of the greatest appeal. The result obtained from $\nu$ smoother iterations with initial guess $\vec{e}$ is denoted by $S\left(A_{l}, \vec{r}_{l}, \vec{e}, \nu\right)$.

The multi-grid algorithm to approximately solve the fine grid problem $A \vec{x}=\vec{b}$ consists of 3 stages. On the first, or "pre" stage, the sequence of problems on the increasingly coarse grids are solved. On the second stage, the problem on the coarsest grid is solved via direct method. On the third, or "past" stage, one solves the sequence of problems on the sequence of the increasingly fine grids. The operation flow of this process is summarized as follows.

\section{Multi-grid algorithm for solving $A \vec{x}=\vec{b}$.}

- BEGIN

- Initialize residual $\vec{r}_{1}$ with initial guess $\vec{x}_{0}$

$$
\vec{r}_{1}=\vec{b}-A_{1} \vec{x}_{0}
$$

- for $l=1,2, \ldots, L-1$ 
* Apply pre-smoother

$$
\vec{e}_{l}=S\left(A_{l}, \vec{r}_{l}, \overrightarrow{0}_{l}, \nu_{\text {pre }}\right)
$$

* Update residual

$$
\vec{r}_{l}=\vec{r}_{l}-A_{l} \vec{e}_{l}
$$

* Project residual on coarser grid

$$
\vec{r}_{l+1}=I_{l}^{\downarrow} \vec{r}_{l}
$$

- end for $l$

- Direct solve on coarsest grid

$$
\vec{e}_{L}=A_{L}^{-1} \vec{r}_{L}
$$

- for $l=L-1, L-2, \ldots, 1$

* Accumulate solution error on finer grid

$$
\vec{e}_{l}=\vec{e}_{l}+I_{l-1}^{\uparrow} \vec{e}_{l-1}
$$

* Apply post-smoother

$$
\vec{e}_{l}=S\left(A_{l}, \vec{r}_{l}, \vec{e}_{l}, \nu_{\text {post }}\right)
$$

- end for $l$

$-\vec{x}=\vec{e}_{1}$

- $E N D$

The computational cost of this algorithm is approximately

$$
4 / 3\left[\nu_{\text {pre }}+\nu_{\text {post }}+1\right][n z(A)]^{p},
$$


where $n z(A)$ is the number of nonzero elements in $A, p$ accounts for the additional cost for preconditioning. For sparse matrices appearing in the MV reconstructor $n z(A)$ is $\mathscr{O}(N)$ and $p$ is around 4/3 for the CG algorithm preconditioners described in [44].

It is also worth mentioning that MG algorithms are very well suitable to parallelization and dedicated hardware implementation, such as Field Programmable Discrete Arrays (FPDA). 
Appendix B.

Computation of the Phase-to-WFS Interaction Matrix With Misalignments Taken into Account

The correct computation of the phase-to-WFS interaction matrix elements with various geometrical misalignments taken into account proves to be the crucial part of the POLC robustness analysis. Matrix $G_{\phi}$ defined in section 3.2 or matrix $G_{a}$ defined in section 3.3 represent the discretized form of WFS measurement operator defined as a WFS measurement is the phase gradient averaged over illuminated part of the subaperture

$$
\left[\begin{array}{c}
s_{x} \\
s_{y}
\end{array}\right]_{i}=\mathscr{M} \phi(x, y)=\frac{1}{\left|W_{i}\right|} \int_{W_{i}} \nabla \phi(x, y) d x d y
$$

where $\phi(x, y)$ is the turbulence phase, $\left|W_{i}\right|=\int_{W_{i}} d x d y$ is the area of the $i^{\text {th }}$ subaperture's illuminated part

$$
W_{i}=W_{a} \cap W_{o} \cap W_{s a}^{i}
$$

$W_{s a}^{i}$ is a square domain of $i^{t h}$ subaperture, $W_{a}, W_{o}$ are the interior of primary and exterior of secondary mirrors, respectively. Approximation of phase with bilinear splines (3.7) turns Eq. (B.1) into

$$
\left[\begin{array}{c}
s_{x} \\
s_{y}
\end{array}\right]_{i} \approx \sum_{j=1}^{M} G_{i j} \phi_{j}
$$

where

$$
G_{i j}=\left[\begin{array}{c}
G_{x} \\
G_{y}
\end{array}\right]_{i j}=\frac{1}{\left|W_{i}\right|} \int_{W_{i}} \nabla f_{j}(x, y) d x d y
$$

Eq. (B.4) gives the form of phase-to-WFS matrix element that needs to be evaluated numerically. It should be noted that no restrictions are applied to the mutual position or sizes of a spline and a subaperture because due to both geometrical misalignments 
and sheer projection of elevated phase screen onto the main aperture the spline and subaperture grids can be arbitrarily shifted and rotated with respect to each other. The most general case of the mutual alignment is shown on Fig. (7.1), where the single spline and subaperture are not only arbitrarily shifted and rotated but also partially barred by the borders of the main annular aperture. This results in quite complex shape of the integration domain $W_{i}$.

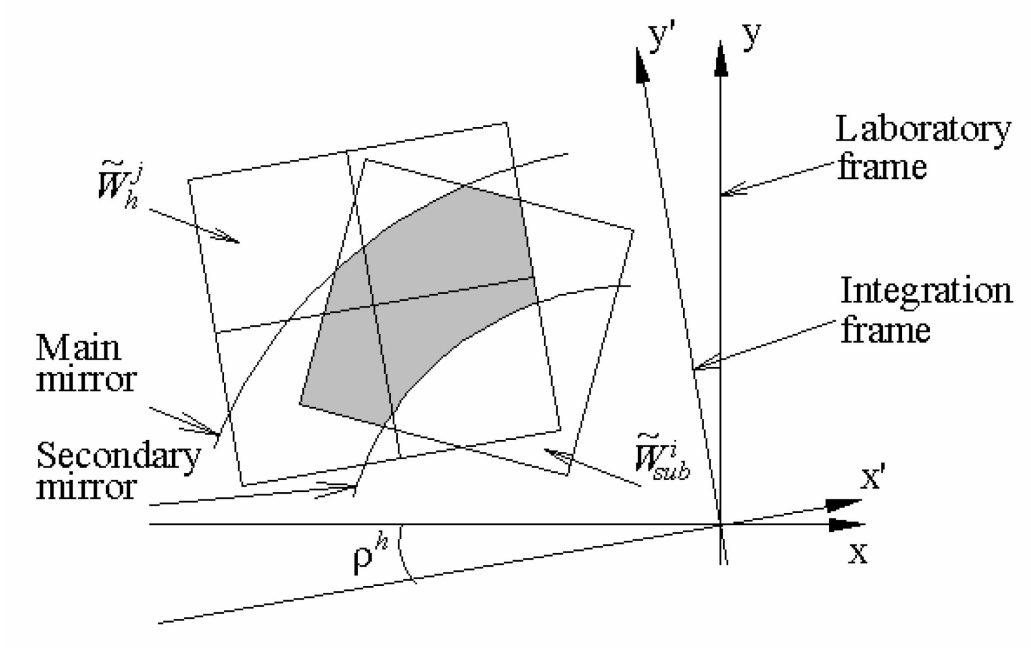

Figure 7.1: Mutual alignment of a WFS subaperture and a bilinear spline. $W_{\text {sub }}^{i}, W_{h}^{j}$ are the domains occupied by a $i^{\text {th }}$ subaperture and $j^{\text {th }}$ spline, respectively

One can consider two methods to evaluate the double integral appearing in Eq. (B.4). First, with the aid of the Stokes theorem the double integral can be turned into a linear one:

$$
G_{i j}=\int_{s_{1}}^{s_{2}} h\left(x(s)-x_{j}, y(s)-y_{j}\right)\left[\begin{array}{c}
d y / d s \\
-d x / d s
\end{array}\right] d s,
$$

where $[x(s), y(s)], s \in\left[s_{1}, s_{2}\right]$ is a parametric representation of the $W_{i}$-domain boundary, which in our case is piecewise continuous. The integral over each piece of the boundary can be found analytically. This gives the fastest way of G-matrix elements evaluation. However, the computation of the segment intersection points is a very te- 
dious process because of the quite complicated shape of the intersection domain (see Fig. 7.1). Another problem of Eq. (B.5) is that the value of derivatives appearing in the integrand can be much larger than the value of the integral itself, which results in severe numerical instability when we try to add up the contributions from each boundary segment.

A more convenient way for numerical implementation of the Eq. (B.4) is to use direct $2 \mathrm{D}$ integration and take advantage of the special form of the integrand to reduce computation complexity. Indeed, we note that the bilinear spline gradient

$$
\nabla f(x, y)=\left[\begin{array}{c}
-\frac{\operatorname{sgn}(x)}{\delta}\left(1-\frac{|y|}{\delta}\right) \\
-\frac{\operatorname{sgn}(y)}{\delta}\left(1-\frac{|x|}{\delta}\right)
\end{array}\right],|x, y| \leq 1
$$

is a linear function on each quadrant, where the bilinear spline is continuous. We also note that the integral of linear function over some rectangular region is

$$
\int_{\square} \nabla f\left(x-x_{0}, y-y_{0}\right) d x d y=\nabla f\left(x_{c}-x_{0}, y_{c}-y_{0}\right) S_{\square},
$$

where $\left(x_{c}, y_{c}\right)$ are the rectangle's center of gravity coordinates, $S_{\square}$ is the rectangle's area. Eq. (B.7) enables to convert double integral into the single one. The intersection region $W_{i}$ is approximated with a set of narrow rectangles as it is shown on Fig. 7.2. Then, using Eq. (B.7) we get for the G-matrix element

$$
G_{i j} \approx \frac{\sum_{k=1}^{N} \nabla f\left(x_{k}-x_{j}, c_{k}-y_{j}\right)\left(b_{k}-a_{k}\right) \Delta x}{\sum_{k=1}^{N}\left(b_{k}-a_{k}\right) \Delta x},
$$

where $a_{k}, b_{k}$ are the $\mathrm{y}$-coordinates of the $k^{\text {th }}$ rectangle central line intersection with the $W_{i}$-domain boundary, $c_{k}=\left(a_{k}+b_{k}\right) / 2$ are the $k^{\text {th }}$ rectangle's center of gravity $\mathrm{y}$-coordinate, $\Delta x$ is the width of the rectangle.

In order to compute the integral it is necessary to find intersections of lines $x=x_{k}$ with the domain $W_{i}$. Using the properties of set intersections we have 


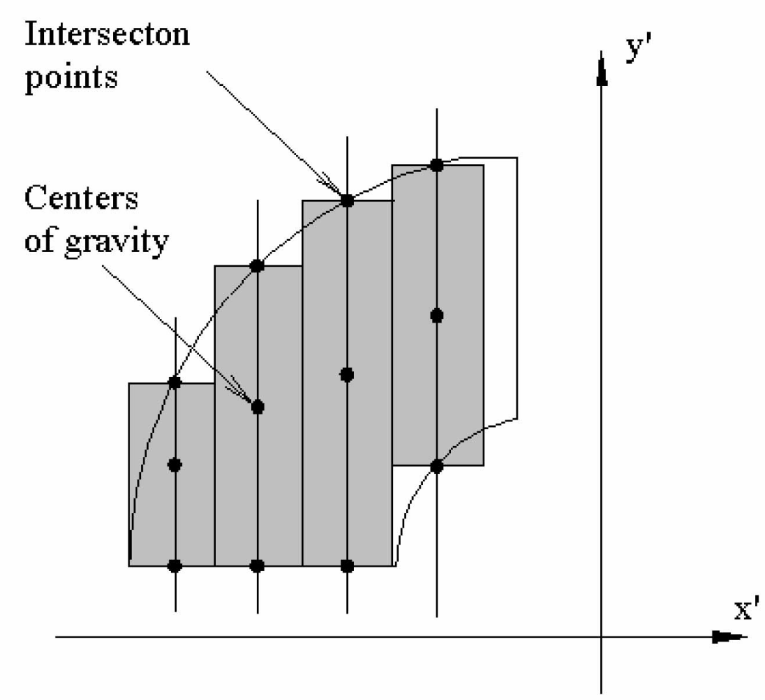

Figure 7.2: Illustration of the numerical integration method.

$$
\begin{gathered}
\left(x=x_{k}\right) \cap W_{i}=\left(x=x_{k}\right) \cap\left(W_{a} \cap W_{o} \cap W_{s a}^{i}\right) \\
=\left(\left(x=x_{k}\right) \cap W_{a}\right) \cap\left(\left(x=x_{k}\right) \cap W_{o}\right) \cap\left(\left(x=x_{k}\right) \cap W_{s a}^{i}\right) .
\end{gathered}
$$

Despite the quite complicated shape of the region $W_{i}$, each of the elementary intersections in Eq. (B.9) is very simple to find. Thus, in the frame of the integration algorithm described the difficult problem of finding the intersection between arbitrary aligned WFS subaperture and bilinear spline turns into a set of simple manipulations that can be easily implemented in computer code.

In order for Eq. (B.7) and, respectively, Eq. (B.8) to be valid we need to operate in the coordinate system oriented along the sides of bilinear spline and also account for geometrical misalignments. This requires the following coordinate transformation

$$
\left[\begin{array}{l}
x \\
y
\end{array}\right]^{\prime}=K\left[\begin{array}{cc}
\cos \left(\theta+\theta_{m}\right) & \sin \left(\theta+\theta_{m}\right) \\
-\sin \left(\theta+\theta_{m}\right) & \cos \left(\theta+\theta_{m}\right)
\end{array}\right]\left[\begin{array}{l}
x \\
y
\end{array}\right]+\left[\begin{array}{c}
\delta x \\
\delta y
\end{array}\right],
$$


where $[x, y]^{T}$ are the spline (subaperture) coordinates in laboratory frame, $K$ is mismagnification factor, $\theta_{m}$ is rotational misalignment angle, $[\delta x, \delta y]$ is a shift misalignment vector, $\theta$ is the spline (subaperture) orientation angle with respect to the coordinate system oriented along spline grid. In order to find the components of $\mathrm{x}$ - and y-slopes in the WFS coordinate system the inverse transformation is done

$$
\left[\begin{array}{c}
G_{x} \\
G_{y}
\end{array}\right]_{i j}=\left[\begin{array}{cc}
\cos \left(\theta_{s p}-\theta_{s a}\right) & -\sin \left(\theta_{s p}-\theta_{s a}\right) \\
\sin \left(\theta_{s p}-\theta_{s a}\right) & \cos \left(\theta_{s p}-\theta_{s a}\right)
\end{array}\right]\left[\begin{array}{c}
G_{x} \\
G_{y}
\end{array}\right]_{i j}^{\prime},
$$

where $\theta_{s p}, \theta_{s a}$ are the spline subaperture grid orientation angles, respectively, in laboratory frame. 


\section{APPENDix C.}

\section{Poles of $Q(z)$.}

We are to find the poles of matrix function

$$
Q(z)=c(z)[I+c(z) M]^{-1}
$$

Matrix $M$ assumes a Jordan canonical factorization

$$
M=S J S^{-1}
$$

where $S$ is some invertible matrix, Jordan form $J$ and its $i^{\text {th }}$ Jordan block are

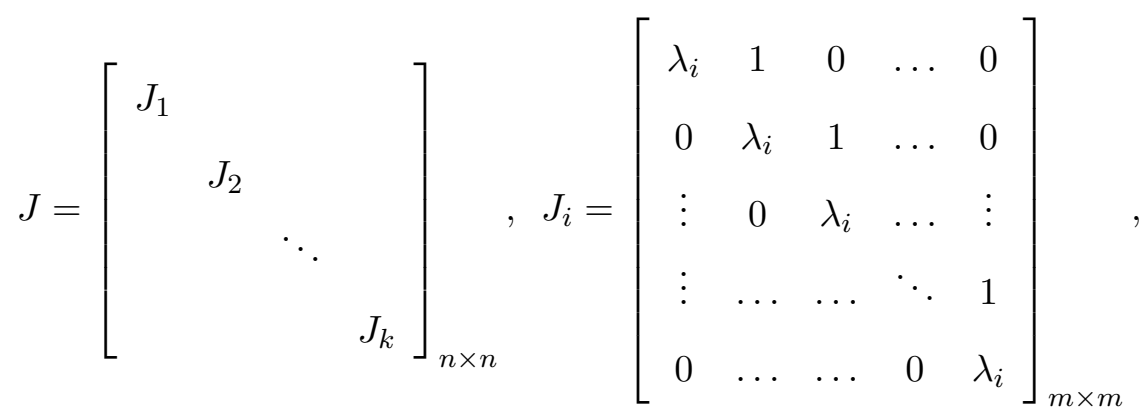

where $k$ is the number of linearly independent eigenvectors of $M, \lambda_{i}$ is one of $M$ 's eigenvalues, $m$ is a geometrical multiplicity of $\lambda_{i}$. With factorization given by Eq. (C.2) matrix $Q(z)$ takes the form

$$
Q(z)=S c(z)[I+c(z) J]^{-1} S^{-1} .
$$

The $i^{\text {th }}$ block of $(I+c(z) J)$ is

$$
B_{i}(z)=\left(1+\lambda_{i} c(z)\right)\left[\begin{array}{ccccc}
1 & \frac{c(z)}{\left(1+\lambda_{i} c(z)\right)} & 0 & \cdots & 0 \\
0 & 1 & \frac{c(z)}{\left(1+\lambda_{i} c(z)\right)} & \ddots & \vdots \\
\vdots & 0 & 1 & \ddots & 0 \\
\vdots & \vdots & \ddots & \ddots & \frac{c(z)}{\left(1+\lambda_{i} c(z)\right)} \\
0 & \cdots & \cdots & 0 & 1
\end{array}\right]
$$


The $B_{i}^{-1}$ can be easily found:

$$
c(z) B_{i}^{-1}=\frac{c(z)}{\left(1+\lambda_{i} c(z)\right)}\left[\begin{array}{ccccc}
1 & -\frac{c(z)}{\left(1+\lambda_{i} c(z)\right)} & \left(\frac{c(z)}{\left(1+\lambda_{i} c(z)\right)}\right)^{2} & \ldots & (-1)^{n}\left(\frac{c(z)}{\left(1+\lambda_{i} c(z)\right)}\right)^{n} \\
0 & 1 & -\frac{c(z)}{\left(1+\lambda_{i} c(z)\right)} & \ldots & \vdots \\
\vdots & 0 & 1 & \ldots & (-1)^{n-1}\left(\frac{c(z)}{\left(1+\lambda_{i} c(z)\right)}\right)^{n-1} \\
\vdots & \ldots & \ldots & \ldots & -\frac{c(z)}{\left(1+\lambda_{i} c(z)\right)} \\
0 & \ldots & \ldots & 0 & 1
\end{array}\right] .
$$

It is obvious from Eq. (C.6) that the poles of matrix $c(z)[I+c(z) J]^{-1}$ and, therefore, of matrix $Q(z)$ are those of functions $c(z) /\left(1+\lambda_{i} c(z)\right)$. 


\section{REFERENCES}

[1] "The California extremely large telescope, conceptual design for a thirty-meter telecope", CELT report 34, (2002), http://tmt.ucolick.org/.

[2] R. Angel, M. Lloyd-Hart, K. Hege, R. Sarlot and C. Peng, "The 20/20 telescope: MCAO imaging at the individual and combined foci," in ESO Conference and Workshop Proceeding Beyond Conventional Adaptive Optics, 58, eds. R. Ragazzoni, N. Hubin, S. Esposito and E. Vernet (Venice, 2002), http://caao.as . arizona.edu/publications/publications/venice2020.pdf, cfa-www.harward. edu/idoc-pub/.

[3] N. M. Milton, M. Lloyd-Hart, A. Cheng, J. A. Georges and R. Angel, "Design and expected performance of an MCAO system for the Giant Magellan Telescope," SPIE Proceedings on Astronomical Adaptive Optics Systems and Applications, 5169, ed. R. K. Tyson and M. Lloyd-Hart (San Diego, 2003).

Home page of GMT: http://www.gmto.org.

[4] Home page of Overwhelmingly Large Telescope: http://www.eso.org/projects/ owl.

[5] G. Rousset, J. C. Fontanella et. al., "First diffraction limited astronomical images with adaptive optics," Assron. and Astrophys., 230, pp. L29 - L32 (1990).

[6] J. Vernin, A. Agabi, R. Avila, Azouit, R. Conan, F. Martin, E. Masciadri, L Sanchez, and A. Ziad, "Gemini CP Site Characterization Report," Internal Report RPT-AO-G0094, Gemini Observatory, January 2000. http://www.gemini. edu/sciops/instruments/adaptiveOptics/AOIndex.html

[7] J. S. Gibson, C.-C. Chang, B. L. Ellerbroek, "Adaptive optics: Wavefront reconstruction by adaptive filtering and control," in 38th IEEE Conference on Decision and Control, (Phoenix, Arizona), IEEE, 1999.

[8] B. L. Ellerbroek, C. Van Loan, N. P. Pitsianis, R. J. Plemmons, "Optimizing closedloop adaptive optics performance with the use of multiple control bandwidths," J. Opt. Soc. Am. A 11 pp. 2871 - 1886 (1994).

[9] B. Le Roux, J. M. Conan, C. Kulcsar, H-F. Raynaud, L. M. Mugnier and T. Fusco, "Optimal control law for multi-conjugate adaptive optics," in Adaptive optical systems technology II, P. L. Wizinowich, ed., Proceedings SPIE 4839, pp. 878 - 889 (2002). 
[10] J. D. Barchers, "Multi-grid approach to predictive wave-front reconstruction in adaptive optical systems," Appl. Opt. 43 (2004).

[11] D. J. MacMartin, "Local, hyerarchic and iterative reconstructors for adaptive optics," J. Opt. Soc. Am. A 20, pp. 1084 - 1093 (2003).

[12] K. Hinnen, M. Verhaegen, N. Doelman, " $\mathscr{H}_{2}$-optimal control of an adaptive optics system: Part I, Data-driven modeling of the wavefront disturbance," Proc. SPIE Int. Soc. Opt. Eng. 5903, pp. 75 - 85 (2005).

[13] K. Hinnen, N. Doelman, M. Verhaegen, " $\mathscr{H}_{2}$-optimal control of an adaptive optics system: Part II, Closed-loop controller design," Proc. SPIE Int. Soc. Opt. Eng. 5903, pp. 86 - 99 (2005).

[14] A. N. Kolmogorov. "The local structure of turbulence in imcompressible viscous fluids for very large Reynolds numbers". In Turbulence, Classic Papers on Statistical Theory, pp. 151 - 155. Wiley-Interscience, New York, 1961.

[15] V. I. Tatarskii, Wave Propagation in a Turbulent Medium. New York, Dover Publications, 1967.

[16] D. L. Fried, "Optical resolution through a randomly inhomogeneous medium for very long and very short exposures," J. Opt. Soc. Am. 56, pp. 1372-1379 (1966).

[17] E. A. Pluzhnik, "Spatial and Temporal Simulations of Atmospheric Phase Distortions," presented on the IPAM conference "Estimation and Control Problems in Adaptive Optics". Los Angeles, January 22 - 24, 2004, http://www.sao.ru/hq/ drabek/ftp/POSTER2.PNG

[18] D. P. Greenwood, "Bandwidth specification for adaptive optics systems," J. Opt. Soc. Am. 67, pp. $174-176$.

[19] J. F. Belsher, D. L. Fried, "Expected antenna gain when correcting tilt-free wavefronts," The Optical Sciences Company, Placentia, CA, Report TR-576.

[20] J. M. Beckers, "Increasing the size of the isoplanatic patch with multi-conjugate adaptive optics," in Proc. European Southern Observatory Conference and Workshop on Very Large Telescopes and their Instrumentation, M. H. Ulrich, ed., 30, pp. 693 - 703 (1988).

[21] D. C. Johnston and B. M. Welsh, "Analysis of multiconjugate adaptive optics," J. Opt. Soc. Am. A 11, pp. 394 - 408 (1994). 
[22] E. Diolaiti, R. Ragazzoni and M. Tordi, "Stability and optimality of a layer oriented MCAO system," in ESO Conference and Workshop Proceeding Beyond Conventional Adaptive Optics, 58, eds. R. Ragazzoni, N. Hubin, S. Esposito and E. Vernet (Venice, 2002).

[23] E. Diolaiti, R. Ragazzoni and M. Tordi, "Closed loop performance of a layeroriented multi-conjugate adaptive optics system," Astron. Astrophys. 372, pp. 710 - 718 (2001).

[24] A. Tokovinin, M. Le Luarn, M. Saracin, "Isoplanatism in a multiconjugate adaptive optics system," J. Opt. Soc. Am. A 17, pp. 1819 - 1827 (2000).

[25] A. Tokovinin, "Maximum separation between guide stars in atmospheric tomography," in ESO Conference and Workshop Proceeding Beyond Conventional Adaptive Optics, 58, eds. R. Ragazzoni, N. Hubin, S. Esposito and E. Vernet (Venice, 2002).

[26] A. Tokovinin, E. Viard, "Limiting precision of tomographic phase estimation," J. Opt. Soc. Am. A 18, pp. 873 - 882 (2001).

[27] L. A. Poyneer, D. T. Gavel, J. M. Brase, "Fast wave-front reconstruction in large adaptive optics systems with use of the Fourier transform," J. Opt. Soc. Am. A 19, pp. 2100 - 2111 (2002).

[28] L. A. Poyneer, J.-P. Veran, "Optimal modal Fourier transform wave-front control," accepted by J. Opt. Soc. Am. A.

[29] B. Macintosh et. al., "Extreme Adaptive Optics Planet Imager: XAOPI," Proc. SPIE Int. Soc. Opt. Eng. 5170, pp. 272 - 282 (2003).

[30] B. L. Ellerbroek, "Linear systems modelling of adaptive optics in the spatial frequency domain," J. Opt. Soc. Am. A 22, pp. 310 - 322 (2005).

[31] R. Hudgin, "Wavefront compensation error due to finite corrector-element size," J. Opt. Soc. Am. 67, pp. 393 - 395 (1977).

[32] B. W. Frazier, R. K. Tyson, M. Smith, J. Roche, "Theory and operation of a robust controller for a compact adaptive optics system," Opt. Eng. 43, pp. 2912 2920 (2004).

[33] F. Roddier, "The effects of atmospheric turbulence in optical astronomy," Progress in Optics XIX, ed. E. Wolf, North-Holland, New York, pp. 283 - 376 (1981).

[34] R. Flicker, F. J. Rigaut and B. L. Ellerbroek, "Comparison of multiconjugate adaptive optics configurations and control algorithms for the Gemini-South $8 \mathrm{~m}$ telescope," in Adaptive optical systems technology, P. L. Wizinowich, ed., Proceedings SPIE 4007, 1032 - 1043 (2000). 
[35] B. L. Ellerbroek, "First-order performance evaluation of adaptive optics systems for atmospheric turbulence compensation in extended-field-of-view astronomical telescopes," J. Opt. Soc. Am. A 11, 783 - 805 (1994).

[36] B. L. Ellerbroek, "Efficient computation of minimum variance wavefront reconstructors using sparse matrix techniques," J. Opt. Soc. Am. A 19, 1803 - 1816 (2002).

[37] N. Roddier, "Atmospheric wavefront simulation using Zernike polynomials," Opt. Eng. 29, pp. 1174 - 1180 (1990).

[38] R. G. Lane, A. Glindemann, J. C. Dainty, "Simulation of a Kolmogorov phase screen," Waves in Random Media 2, pp. 209 - 224 (1992).

[39] A. Glindemann, R. G. Lane, J. C. Dainty, "Simulation of time-evolving speckle patterns using Kolmogorov statistics," J. Mod. Opt. 40, pp. 2381 - 2388 (1993).

[40] C. M. Harding, R. A. Johnston, R. G. Lane, "Fast simulation of a Kolmogorov phase screen," Appl. Opt. 38, pp. 2161 - 2170 (1999).

[41] AOTools, the optical sciences company. www.tosc.com.

[42] Plancherel's theorem: www.mathworld.wolfram.com/PlancherelsTheorem. html.

[43] L. Gilles, C. R. Vogel, B. L. Ellerbroek, "Multigrid preconditioned conjugategradient method for large-scale ave-front reconstruction," J. Opt. Soc. Am. A 19 pp. 1803 - 1816 (2002).

[44] L. Gilles, B. L. Ellerbroek, C. R. Vogel, "Preconditioned conjugate gradient wavefront reconstructors for multi-conjugate adaptive optics", Appl. Opt. 42, 5233 - 5250 (2003).

[45] C. R. Vogel, Q. Yang, "Multigrid algorithm for least squares wavefront reconstruction," accepted by Appl. Opt.

[46] L. Gilles, "Closed-loop stability and performance analysis of least-squares and minimum variance control algorithms for multi conjugate adaptive optics," Appl. Opt. 44, pp. 993 - 1003 (2005).

[47] B. L. Ellerbroek and C. R. Vogel, "Simulations of closed-loop wavefront reconstruction for multi-conjugate adaptive optics on giant telescopes," Proc. SPIE 5169, pp. 206 - 217 (2003).

[48] B. L. Ellerbroek, "Wavefront reconstruction algorithms and simulation results for multi-conjugate adaptive optics on giant telescopes," Proc. SPIE 5382 (2003). 
[49] P. Piatrou, "Phase-to-WFS influence matrix computations cross-verification," presented on the IPAM conference "Estimation and Control Problems in Adaptive Optics". Los Angeles, January 22 - 24, 2004, http://www.ipam.ucla.edu/ publications/ao2004/ao_2004_4636.pdf

[50] P. Piatrou, L. Gilles, "Robustness study of the pseudo open-loop controller for multiconjugate adaptive optics," Appl. Opt. 44, pp. 1003 - 1010 (2005).

[51] T. Fusco, J.-M. Conan, G. Rousset, L. M. Mugnier, V. Michau, "Optimal wavefront reconstruction strategies for multiconjugate adaptive optics," J. Opt. Soc. Am. A 18, pp. 2527 - 2538 (2001).

[52] E. D. Wallner, "Optimal wave-front correction using slope measurements," J. Opt. Soc. Am. 73, pp. 1771 - 1776 (1983).

[53] D. G. Hoelscher, Adaptive Estimation of Optical Aberrations Due To Atmospheric Turbulence, MS thesis, Texas Tech. Univ. (1979).

[54] R. N. Paschall, D. J. Anderson, "Linear quadratic Gaussian control of deformable mirror adaptive optics system with time delayed measurements," Appl. Opt. 32, pp. 6347 - 6357 (1993).

[55] D. M. Wiberg, C. E. Max, D. T. Gavel, "A geometric view of adaptive optics control," Proceedings SPIE (2004).

[56] D. T. Gavel, D. M. Wiberg, "Towards strehl-optimizing adaptive optics controllers," in Adaptive optical systems technology II, P. L. Wizinowich, ed., Proceeding SPIE 4839, pp. 890 - 901 (2002).

[57] B. Le Roux, J. M. Conan, C. Kulcsar, H-F. Rayand, L. M. Mugnier and T. Fusco, "Optimal control low for multi-conjugate adaptive optics," in Adaptive optical systems technology II, P. L. Wizinovich, ed., Proceedings SPIE 4939, pp. 878 - 889 (2002).

[58] P. Piatrou, M. C. Roggemann, "Performance analysis of Kalman filter and minimum variance controllers for multi conjugate adaptive optics," Proc. SPIE Int. Soc. Opt. Eng. 5894, 5894OX (2005).

[59] "MCAO Conceptual Design Review Documents for Gemini-South," Gemini Observatory (2000), www.gemini.edu/sciops/instruments/adaptiveOptics/A0Index. html.

[60] J. W. Goodman, Statistical Optics, New York: John Wiley \& Sons, 1985. 
[61] D. W. Hardy, Adaptive optics for astronomical telescopes, Oxford University Press, 1998.

[62] M. C. Roggemann, B. M. Welsh, Imaging through turbulence, CRC Press, 1996.

[63] J. T. Beyer, M. C. Roggemann, L. J. Otten, T. J. Schulz, T. C. Havens, W. Brown, "Experimental estimation of the spatial statistics of turbulence-induced index of refraction fluctuations in the upper atmosphere," Appl. Opt. 42, pp. 908 - 921 (2003).

[64] L. C. Ludeman, Random processes: Filtering, Estimation and Detection, Wiley, 2003.

[65] M. D. Srinath, P. K. Rajasekaran, R. Viswanathan, Introduction to statistical signal processing with applications, Prentice Hall, 1996.

[66] J. B. Burl, Linear optimal Control: $\mathscr{H}_{2}$ and $\mathscr{H}_{\infty}$ Methods, Addison-Wesley (1998).

[67] G. H. Golub, C. F. van Loan, Matrix Computations, Johns Hopkins University Press (1996).

[68] L. N. Trefethen, D. Bau III, Numerical Linear Algebra, SIAM (1997).

[69] U. Trottenberg, C. W. Oosterlee, A. Schuller, Multigrid, Academic Press, London (2001).

[70] G. A. Korn, T. M. Korn, Mathematical handbook for scientists and ingineers. 2nd ed., McGraw-Hill (1968).

[71] P. M. Clarkson, Optimal and Adaptive Signal Processing, CRC Press (1993).

[72] J. G. Proakis et. al., Algorithms for statistical signal processing, Prentice Hall (2002). 